\title{
Should Dithiophosphinate Esters Function As RAFT Agents?
}

\author{
Jennifer L. Hodgson, Katy A. Green, and Michelle L Coote* \\ Research School of Chemistry, Australian National University, Canberra, \\ ACT 0200, Australia, mcoote@rsc.anu.edu.au
}

SUPPORTING INFORMATION 


\section{Computational Procedures}

Standard ab initio molecular orbital theory and density functional theory calculations were performed using GAUSSIAN $03^{1}$ and Molpro $2000.6^{2}$ software. Barriers and enthalpies (0 K, kJ $\mathrm{mol}^{-1}$ ) were calculated for methyl radical addition to the sulfur center of various dithiophosphinate ester analogues of RAFT agents $\left(\mathrm{S}=\mathrm{P}(\mathrm{Z})\left(\mathrm{Z}^{\prime}\right) \mathrm{SCH}_{3} ; \mathrm{Z}, \mathrm{Z}^{\prime}=\mathrm{CH}_{3}, \mathrm{CN}, \mathrm{OCH}_{3}\right.$ and $\mathrm{Ph}$ ) and also for the simpler system, $\mathrm{S}=\mathrm{P}\left(\mathrm{CH}_{3}\right)_{3}$. Corresponding barriers and enthalpies for the standard RAFT agents (i.e. $\mathrm{S}=\mathrm{C}(\mathrm{Z}) \mathrm{SCH}_{3}$ and $\mathrm{S}=\mathrm{C}\left(\mathrm{CH}_{3}\right)_{2}$ ) were either taken from earlier studies $^{3}$ at the same level of theory, or calculated as part of the present work. Calculations were performed at a high level of theory chosen on the basis of a recent assessment studies for radical addition to $\mathrm{C}=\mathrm{S}$ bonds, both in prototypical systems and also RAFT-related systems. ${ }^{3 \mathrm{a}, \mathrm{d}, 4}$ Geometries of the reactants, products and transition structures were optimized at the B3-LYP/631G(d) level of theory and improved energies were calculated using G3(MP2)-RAD. ${ }^{5}$ Radical stabilization energies (RSEs) of the phosphorus-centered and carbon-centered radical products of these reactions were also calculated at the G3(MP2)-RAD//B3-LYP/6-31G(d) level of theory. For the purposes of the present work, the RSEs of all radicals $(\mathrm{R} \bullet)$ were calculated relative to the same reference species $\left(\bullet \mathrm{CH}_{3}\right)$ and were defined as the energy change of the reaction: $\bullet \mathrm{R}+\mathrm{CH}_{4}$ $\rightarrow \mathrm{R}-\mathrm{H}+\cdot \mathrm{CH}_{3}$.

To assist in the qualitative rationalization of the results, the vertical singlet-triplet excitation energies of the prototypical systems, $\mathrm{S}=\mathrm{P}\left(\mathrm{CH}_{3}\right)_{2} \mathrm{SCH}_{3}$ and $\mathrm{S}=\mathrm{C}\left(\mathrm{CH}_{3}\right) \mathrm{SCH}_{3}$, were calculated at the G3(MP2)-RAD level of theory, and the charge distributions within the ground state species were calculated via an NBO population analysis, carried out using the B3-LYP/6-311+G(3df,2p) density. The strengths of the $\mathrm{C}=\mathrm{S}$ and $\mathrm{P}=\mathrm{S}$ double bonds in $\mathrm{S}=\mathrm{P}\left(\mathrm{CH}_{3}\right)_{2} \mathrm{SCH}_{3}$ and $\mathrm{S}=\mathrm{C}_{\left(\mathrm{CH}_{3}\right) \mathrm{SCH}_{3}}$ were calculated using the thermodynamic cycle described by Schmidt and co-workers. ${ }^{6}$ The double bond energies were obtained by correcting the energies for their destruction via hydrogenation reactions $\left(\Delta \mathrm{H}_{\text {hyd }}\right)$ with the energy of the $\mathrm{H}-\mathrm{H}$ bond $\left(\mathrm{D}_{\mathrm{H}-\mathrm{H}}\right)$ that is also destroyed, and the energies of the $\mathrm{S}-\mathrm{H}\left(\mathrm{D}_{\mathrm{S}-\mathrm{H}}\right), \mathrm{C}-\mathrm{H}\left(\mathrm{D}_{\mathrm{C}-\mathrm{H}}\right)$ and/or $\mathrm{P}-\mathrm{H}\left(\mathrm{D}_{\mathrm{P}-\mathrm{H}}\right)$ sigma bonds that are formed, in the same process. All calculations were performed at the G3(MP2)-RAD//B3-LYP/6-31G(d) level of theory. The full calculations for $\mathrm{S}=\mathrm{P}\left(\mathrm{CH}_{3}\right)_{2} \mathrm{SCH}_{3}$ and $\mathrm{S}=\mathrm{C}\left(\mathrm{CH}_{3}\right) \mathrm{SCH}_{3}$ are as follows: 


$$
\begin{aligned}
& D_{P=S}=\Delta \mathrm{H}_{\mathrm{hyd}}-D_{H-H}+D_{P-H}+D_{S-H} \\
& \text { where: } \\
& \mathrm{S}=\mathrm{P}\left(\mathrm{CH}_{3}\right)_{2} \mathrm{SCH}_{3}+\mathrm{H}_{2} \rightarrow \mathrm{HS}-\mathrm{PH}\left(\mathrm{CH}_{3}\right)_{2} \mathrm{SCH}_{3} \quad \Delta \mathrm{H}_{\text {hyd }} \\
& 2 \mathrm{H} \bullet \rightarrow \mathrm{H}_{2} \rightarrow-\mathrm{D}_{\mathrm{H}-\mathrm{H}} \\
& \mathrm{HS}-\mathrm{PH}\left(\mathrm{CH}_{3}\right)_{2} \mathrm{SCH}_{3} \rightarrow \mathrm{HS}-\mathrm{P} \bullet\left(\mathrm{CH}_{3}\right)_{2} \mathrm{SCH}_{3}+\mathrm{H} \bullet \quad \mathrm{D}_{\mathrm{P}-\mathrm{H}} \\
& \mathrm{HS}-\mathrm{PH}\left(\mathrm{CH}_{3}\right)_{2} \mathrm{SCH}_{3} \rightarrow \bullet \mathrm{S}-\mathrm{PH}\left(\mathrm{CH}_{3}\right)_{2} \mathrm{SCH}_{3}+\mathrm{H} \bullet \quad \mathrm{D}_{\mathrm{S}-\mathrm{H}} \\
& D_{C=S}=\Delta H_{\mathrm{hyd}}-D_{H-H}+D_{C-H}+D_{S-H} \\
& \text { where: } \\
& \begin{array}{ll}
\mathrm{S}=\mathrm{C}\left(\mathrm{CH}_{3}\right) \mathrm{SCH}_{3}+\mathrm{H}_{2} \rightarrow \mathrm{HS}-\mathrm{CH}\left(\mathrm{CH}_{3}\right) \mathrm{SCH}_{3} & \Delta \mathrm{H}_{\mathrm{hyd}} \\
2 \mathrm{H} \bullet \quad \rightarrow \mathrm{H}_{2} & -\mathrm{D}_{\mathrm{H}-\mathrm{H}} \\
\mathrm{HS}-\mathrm{CH}\left(\mathrm{CH}_{3}\right) \mathrm{SCH}_{3} \rightarrow \bullet \mathrm{S}-\mathrm{CH}\left(\mathrm{CH}_{3}\right) \mathrm{SCH}_{3}+\mathrm{H} \bullet & \mathrm{D}_{\mathrm{S}-\mathrm{H}} \\
\mathrm{HS}-\mathrm{CH}\left(\mathrm{CH}_{3}\right) \mathrm{SCH}_{3} \rightarrow \mathrm{HS}-\mathrm{C} \bullet\left(\mathrm{CH}_{3}\right) \mathrm{SCH}_{3}+\mathrm{H} \bullet & \mathrm{D}_{\mathrm{C}-\mathrm{H}}
\end{array}
\end{aligned}
$$

1 Frisch, M. J.; Trucks, G. W.; Schlegel, H. B.; Scuseria, G. E.; Robb, M. A.; Cheeseman, J. R.; Montgomery Jr., J. A.; Vreven, T.; Kudin, K. N.; Burant, J. C.; Millam, J. M.; Iyengar, S. S.; Tomasi, J.; Barone, V.; Mennucci, B.; Cossi, M.; Scalmani, G.; Rega, N.; Petersson, G. A.; Nakatsuji, H.; Hada, M.; Ehara, M.; Toyota, K.; Fukuda, R.; Hasegawa, J.; Ishida, M.; Nakajima, T.; Honda, Y.; Kitao, O.; Nakai, H.; Klene, M.; Li, X.; Knox, J. E.; Hratchian, H. P.; Cross, J. B.; Adamo, C.; Jaramillo, J.; Gomperts, R.; Stratmann, R. E.; Yazyev, O.; Austin, A. J.; Cammi, R.; Pomelli, C.; Ochterski, J. W.; Ayala, P. Y.; Morokuma, K.; Voth, G. A.; Salvador, P.; Dannenberg, J. J.; Zakrzewski, V. G.; Dapprich, S.; Daniels, A. D.; Strain, M. C.; Farkas, O.; Malick, D. K.; Rabuck, A. D.; Raghavachari, K.; Foresman, J. B.; Ortiz, J. V.; Cui, Q.; Baboul, A. G.; Clifford, S.; Cioslowski, J.; Stefanov, B. B.; Liu, G.; Liashenko, A.; Piskorz, P.; Komaromi, I.; Martin, R. L.; Fox, D. J.; Keith, T.; Al-Laham, M. A.; Peng, C. Y.; Nanayakkara, A.; Challacombe, M.; Gill, P. M. W.; Johnson, B.; Chen, W.; Wong, M. W.; Gonzalez, C.; Pople, J. A. Gaussian 03, Revision B.03; Gaussian, Inc.: Pittsburgh PA, 2003.

2 Werner, H.-J.; Knowles, P. J.; Amos, R. D.; Bernhardsson, A.; Berning, A.; Celani, P.; Cooper, D. L.; Deegan, M. J. O.; Dobbyn, A. J.; Eckert, F.; Hampel, C.; Hetzer, G.; Korona, T.; Lindh, R.; Lloyd, A. W.; McNicholas, S. J.; Manby, F. R.; Meyer, W.; Mura, M. E.; Nicklass, A.; Palmieri, P.; Pitzer, R.; Rauhut, G.; Schütz, M.; Stoll, H.; Stone, A. J.; Tarroni, R.; Thorsteinsson, T. MOLPRO 2000.6; University of Birmingham: Birmingham, 1999

3 (a) Coote, M. L.; Wood, G. P. F.; Radom, L. J. Phys. Chem. A 2002, 106, 12124-12138. (b) Coote, M. L.; Radom, L. J. Am. Chem. Soc. 2003, 125, 1490-1491. (c) Coote, M. L.; Radom, L. Macromolecules 2004, 37, 590-596. (d) Coote, M. L.; Henry, D. J. Macromolecules 2005, 38, 1415-1433. (e) Hodgson, J. L.; Coote, M. L. J. Phys. Chem. A, 2005, submitted.

4 Coote, M. L. J. Phys. Chem. A 2005, 109, 1230-1239.

5 Henry, D. J.; Sullivan, M. B.; Radom, L. J. Chem. Phys. 2003, 118, 4849-4860.

6 Schmidt, M. W.; Truong, P. H.; Gordon, M. S. J. Am. Chem. Soc. 1987, 109, 5217-27. 
Table S1. B3-LYP/6-31G(d) Optimized Geometries

\author{
$P=S$ Additions
}

\begin{abstract}
$\cdot \mathrm{CH}_{3}$

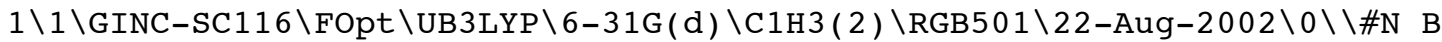
3LYP 6-31G(D) SCF=TIGHT OPT=TIGHT FREQ MAXDISK=13107200\\methyl Opt\&Fr eq UB3-LYP $/ 6-31 \mathrm{G}(\mathrm{d}) \backslash \backslash 0,2 \backslash \mathrm{C}, 0 ., 0 ., 0 . \backslash \mathrm{H}, 1.082752351,0 ., 0 . \backslash \mathrm{H},-0.541376175$ $5,-0.937691042,0 . \backslash \mathrm{H},-0.5413761755,0.937691042,0 . \backslash \backslash$ Version=DEC-AXP-OSF/ $1-\mathrm{G} 98 \mathrm{RevA} .11 .3 \backslash \mathrm{HF}=-39.8382922 \backslash \mathrm{S} 2=0.753765 \backslash \mathrm{S} 2-1=0 . \backslash \mathrm{S} 2 \mathrm{~A}=0.750007 \backslash \mathrm{RMSD}=4$. $410 \mathrm{e}-09 \backslash \mathrm{RMSF}=1.426 \mathrm{e}-09 \backslash \mathrm{Dipole}=0 ., 0 ., 0 . \backslash \mathrm{PG}=\mathrm{D} 03 \mathrm{H} \quad[\mathrm{O}(\mathrm{C} 1), 3 \mathrm{C} 2(\mathrm{H} 1)] \backslash \backslash @$
\end{abstract}

\title{
$\mathrm{S}=\mathrm{P}\left(\mathrm{CH}_{3}\right)_{2} \mathrm{SCH}_{3}$
}

$1 \backslash 1 \backslash G I N C-L C 86 \backslash F O p t \backslash R B 3 L Y P \backslash 6-31 G(d) \backslash C 3 H 9 P 1 S 2 \backslash M L C 501 \backslash 05-J u l-2004 \backslash 0 \backslash \backslash \#$ B3

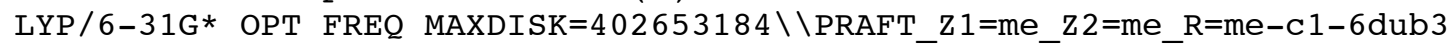
$\backslash \backslash 0,1 \backslash S, 0.5757253594,-1.7469428194,0.7829922 \overline{0} 9 \backslash \mathrm{P}, 0.42221 \overline{1} 8195,-0.15642$ $54872,-0.3640552076 \backslash \mathrm{S},-1.3169499424,1.0746166451,-0.0703360662 \backslash \mathrm{C}, 1.860$ $0775992,0.986204391,-0.2443423182 \backslash \mathrm{C}, 0.2642268131,-0.4856053393,-2.1637$ $910161 \backslash \mathrm{C},-1.5737239165,0.916761274,1.7401124353 \backslash \mathrm{H}, 1.1771324991,-0.9800$ $506718,-2.5091142454 \backslash \mathrm{H}, 0.1199769558,0.4467490189,-2.7202610901 \backslash \mathrm{H},-0.58$ $7523329,-1.1485331686,-2.3310496551 \backslash \mathrm{H}, 2.7682337934,0.4425209126,-0.523$ $2939696 \backslash \mathrm{H}, 1.9604583292,1.3295617753,0.7882985003 \backslash \mathrm{H}, 1.7216874134,1.8487$ $723814,-0.9034586505 \backslash \mathrm{H},-0.905702857,1.5768078874,2.297383685 \backslash \mathrm{H},-1.4202$ $45126,-0.1219993725,2.0438404923 \backslash \mathrm{H},-2.6110846193,1.2056103797,1.924110$ $1573 \backslash \backslash$ Version $=x 86-$ Linux-G03RevB.03 \State $=1-A \backslash H F=-1257.5189656 \backslash$ RMSD $=4.0$ $11 \mathrm{e}-09 \backslash \mathrm{RMSF}=1.869 \mathrm{e}-05 \backslash \mathrm{Dipole}=0.2500109,1.1326532,-0.5970137 \backslash \mathrm{PG}=\mathrm{C} 01] \mathrm{X}($ $\mathrm{C} 3 \mathrm{H} 9 \mathrm{P} 1 \mathrm{~S} 2) \mathrm{J} \backslash \backslash \mathrm{Q}$

\section{TS: $\cdot \mathrm{CH}_{3}+\mathrm{S}=\mathrm{P}\left(\mathrm{CH}_{3}\right)_{2} \mathrm{SCH}_{3}$}

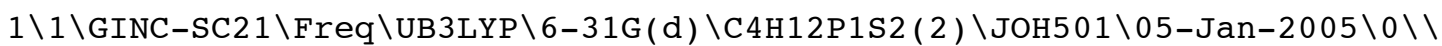
\# B3LYP/6-31G* OPT=(TS, CALCALL, NOEIGENTEST, MAXCYC $=200)$ MAXDISK $=1342177$ $28 \backslash \backslash t s$ tch3spme2sch3_up1 gaussian 98 transition state search $\backslash \backslash 0,2 \backslash S,-1$. $619373 \overline{9} 475,-0.34671 \overline{0} 6129,-0.0326468812 \backslash \mathrm{P}, 0.4150142339,-0.2899446938,-0$ $.0072075303 \backslash \mathrm{S}, 1.5550141789,1.5533955001,-0.3650165758 \backslash \mathrm{C}, 0.4365262806,2$ $.7691453058,0.4393889826 \backslash \mathrm{C}, 1.1012540847,-0.9870349487,1.5568336865 \backslash \mathrm{C}, 1$ $.1636696079,-1.3411922074,-1.3171817258 \backslash \mathrm{H}, 0.6934432047,3.7468251015,0$. $0251100554 \backslash \mathrm{H},-0.6047925532,2.5359810939,0.2009599134 \backslash \mathrm{H}, 0.5773752859,2$. $7863702059,1.5225191466 \backslash \mathrm{H}, 0.8454693082,-2.377336201,-1.1692565506 \backslash \mathrm{H}, 2$. $2567009257,-1.2869307608,-1.2795485326 \backslash \mathrm{H}, 0.8153089464,-0.9947477126,-2$ $.2929122061 \backslash \mathrm{H}, 0.7190410471,-2.0027434854,1.6983406939 \backslash \mathrm{H}, 0.7763498329,-$ $0.3687062915,2.3975537164 \backslash \mathrm{H}, 2.1945635791,-1.0019719421,1.5188653288 \backslash \mathrm{C}$, $-3.2409779334,-2.0212887151,-0.0250886337 \backslash \mathrm{H},-4.1202028594,-1.382044773$ $1,-0.0261916257 \backslash \mathrm{H},-3.0556052054,-2.5664933501,-0.9451890007 \backslash \mathrm{H},-3.05594$ $09621,-2.5637662809,0.8967634704 \backslash \backslash$ Version=DEC-AXP-OSF / 1-G03RevB.03\Sta $\mathrm{te}=2-\mathrm{A} \backslash \mathrm{HF}=-1297.3462727 \backslash \mathrm{S} 2=0.766238 \backslash \mathrm{S} 2-1=0 . \backslash \mathrm{S} 2 \mathrm{~A}=0.750082 \backslash \mathrm{RMSD}=7.432 \mathrm{e}-0$ $9 \backslash \mathrm{RMSF}=2.086 \mathrm{e}-06 \backslash \mathrm{Dipole}=0.7051087,-0.5307035,0.3842953 \backslash \mathrm{DipoleDeriv}=0.0$ $24377,0.5352675,-0.0087911,0.5531081,0.017154,-0.0064547,-0.0174115,-0$ $.0087148,-0.4481461,1.6691349,0.3576297,0.0057,0.162938,1.2594422,-0.0$ $652465,-0.1103386,-0.1592202,0.8895602,-0.9738553,-0.6531123,0.0309137$ $,-0.5704272,-0.8884939,0.0146479,0.1099676,0.1503895,-0.2643836,0.0323$ $89,-0.1492186,-0.0655399,0.01227,0.2113312,0.076704,0.0364967,0.188789$ $1,0.1228759,-0.2443916,0.0162504,-0.0846604,0.1479348,-0.1962012,0.080$ $6866,-0.0137435,0.0049849,-0.2550677,-0.2269686,0.0593919,0.063719,0.0$ $793831,-0.2507385,-0.0716836,-0.0049023,0.0050515,-0.2385381,0.0772216$ $, 0.0053548,0.0329036,-0.0059318,-0.1004513,0.0192324,0.0258122,-0.0115$ $606,0.0562841,-0.0112816,0.0839502,0.0038463,0.013637,0.0918608,-0.027$ $9701,-0.0133112,-0.0869065,0.0427251,0.048061,0.0826741,0.0268387,0.03$ $35899,0.0014359,-0.0309928,-0.0018642,-0.0919068,-0.1145888,0.0521099$, $-0.0239066,0.0227053,-0.0162913,-0.0624927,0.003451,0.024172,-0.021606$ $3,0.0623217,-0.082741,0.009773,0.0097755,0.0204526,0.0721024,-0.024006$ $5,0.0362444,-0.0270122,0.055431,0.0669894,0.0123473,-0.0113652,-0.0031$ 
$792,0.0681999,0.0087193,-0.0313868,0.0326472,-0.0255429,0.0261655,-0.0$ $427405,-0.0227192,-0.0227054,-0.0522986,0.0371043,-0.0331602,0.0550469$ $, 0.040855,0.0694074,0.0211809,0.0134809,0.0062307,0.0433289,-0.0453168$ $, 0.0425001,-0.0535047,-0.0200071,-0.0857809,0.016651,-0.0183599,0.0326$ $608,0.0761122,0.0315018,-0.0536077,0.0189244,0.0317709,-0.3116131,-0.2$ $691874,0.001997,-0.3237033,-0.1850773,-0.0006861,0.0026163,0.0039306,0$ $.0935801,-0.0932507,0.022325,0.0004267,-0.0209559,-0.0226345,-0.000001$ $2,0.0007911,0.0001738,0.0517345,-0.0170101,-0.0413144,0.0082627,-0.048$ $9933,-0.0404373,-0.0646328,0.0192788,-0.0495859,-0.0390284,-0.0189627$, $-0.0433161,-0.0091337,-0.0500174,-0.0421423,0.0649439,-0.0181531,0.050$ $0801,-0.041836 \backslash$ Polar $=157.8035263,43.1786038,132.3828508,-3.2129493,1.2$ $640261,80.4119684 \backslash \mathrm{PG}=\mathrm{C} 01 \quad[\mathrm{X}(\mathrm{C} 4 \mathrm{H} 12 \mathrm{P} 1 \mathrm{~S} 2)] \backslash \mathrm{NImag}=1 \backslash \backslash$

\section{$\mathrm{CH}_{3} \mathrm{SP} \cdot\left(\mathrm{CH}_{3}\right)_{2} \mathrm{SCH}_{3}$}

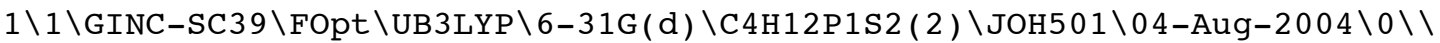
\# B3LYP/6-31G* OPT MAXDISK=134217728\\ch3spme2sch3 2 optimisation b3ly $\mathrm{p} \backslash \backslash 0,2 \backslash \mathrm{S},-2.1540746907,-0.317603516,-0.6373994315 \backslash \mathrm{P}, 0.1053684513,-0.35$ $26199144,-0.1719444253 \backslash S, 2.1634048987,0.6823837536,-0.0376972373 \backslash \mathrm{C},-2$. $7006439948,0.9211033206,0.6006984449 \backslash \mathrm{C}, 1.7806469275,1.9587159987,1.223$ $3945886 \backslash \mathrm{C}, 0.211796087,-1.6246011729,1.1837047261 \backslash \mathrm{C}, 0.5433241001,-1.342$ $300917,-1.6742636818 \backslash \mathrm{H},-3.7629140969,1.1023510738,0.4172992015 \backslash \mathrm{H},-2.15$ $88355545,1.8624854075,0.4784508711 \backslash \mathrm{H},-2.5790797394,0.5546844147,1.6236$ $825565 \backslash \mathrm{H},-0.1712494974,-2.1594774093,-1.8077326727 \backslash \mathrm{H}, 1.5489926658,-1.7$ $593207398,-1.5696712944 \backslash \mathrm{H}, 0.514520715,-0.6949574232,-2.5543762748 \backslash \mathrm{H},-0$ $.5055515089,-2.4276313945,0.9941821809 \backslash \mathrm{H},-0.027446652,-1.1604567358,2$. $1452255198 \backslash \mathrm{H}, 1.2266700463,-2.0283912378,1.2326033198 \backslash \mathrm{H}, 1.5701355574,1$. $5150281425,2.200314736 \backslash \mathrm{H}, 0.9369580499,2.5787619673,0.909592547 \backslash \mathrm{H}, 2.667$ $2511984,2.5922354738,1.3099379225 \backslash \backslash$ Version=DEC-AXP-OSF / 1-G03RevB.03\St ate $=2-\mathrm{A} \backslash \mathrm{HF}=-1297.3750794 \backslash \mathrm{S} 2=0.757435 \backslash \mathrm{S} 2-1=0 . \backslash \mathrm{S} 2 \mathrm{~A}=0.750031 \backslash \mathrm{RMSD}=6.004 \mathrm{e}-$ $09 \backslash \mathrm{RMSF}=6.206 \mathrm{e}-06 \backslash \mathrm{Dipole}=-0.0853044,-0.1027258,0.7815209 \backslash \mathrm{PG}=\mathrm{C} 01 \quad[\mathrm{X}(\mathrm{C} 4 \mathrm{H}$ $12 \mathrm{P} 1 \mathrm{~S} 2) \mathrm{]} \backslash \backslash \mathrm{Q}$

\section{$\mathrm{CH}_{3} \mathrm{SP}(\mathrm{H})\left(\mathrm{CH}_{3}\right)_{2} \mathrm{SCH}_{3}$}

$1 \backslash 1 \backslash G I N C-S C 32 \backslash F O p t \backslash R B 3 L Y P \backslash 6-31 G(d) \backslash C 4 H 13 P 1 S 2 \backslash J O H 501 \backslash 09-S e p-2004 \backslash 0 \backslash \backslash \# B$ 3LYP/6-31G* OPT MAXDISK=134217728 MAXDISK $=134217728 \backslash \backslash a \_c h 3 s p m e 2 s c h 3$ 2 optimisation b3lyp $\backslash \backslash 0,1 \backslash S,-2.2457133153,-0.4329350961,-0.3347901883 \backslash P$, $0.0481875174,-0.1905632985,-0.2135698641 \backslash S, 2.1905082734,0.6646672061,-$ $0.3300761242 \backslash \mathrm{C},-2.7434450251,0.4102847294,1.2087512516 \backslash \mathrm{C}, 2.2324670949$, $1.6502444119,1.2088118899 \backslash \mathrm{C}, 0.3949386779,-1.6212414726,0.8941672135 \backslash \mathrm{C}$, $0.1673355317,-0.6634274913,-1.9993486748 \backslash \mathrm{H},-3.7464240967,0.8205526362$, $1.068139287 \backslash \mathrm{H},-2.0657120548,1.2470040263,1.4325851587 \backslash \mathrm{H},-2.7559647698$, $-0.2723521247,2.063380096 \backslash \mathrm{H},-0.5675782216,-1.4421913807,-2.2202252776 \backslash$ $\mathrm{H}, 1.1804889738,-1.0119012391,-2.2171563193 \backslash \mathrm{H},-0.0484967726,0.218199785$ $5,-2.6065815282 \backslash \mathrm{H},-0.3232779765,-2.4192323154,0.6941722542 \backslash \mathrm{H}, 0.2745438$ $706,-1.2924433996,1.9309097104 \backslash \mathrm{H}, 1.4221840905,-1.9612486418,0.74755053$ $26 \backslash \mathrm{H}, 2.5622437744,1.0573907291,2.0668867653 \backslash \mathrm{H}, 1.2410795715,2.072359013$ $5,1.4290933121 \backslash \mathrm{H}, 2.9260401459,2.4823336995,1.0651364282 \backslash \mathrm{H},-0.246436301$ $9,0.9971038637,0.5132284612 \backslash \backslash$ Version=DEC-AXP-OSF / 1-G03RevB .03 \State=1$\mathrm{A} \backslash \mathrm{HF}=-1297.9670361 \backslash \mathrm{RMSD}=3.469 \mathrm{e}-09 \backslash \mathrm{RMSF}=3.086 \mathrm{e}-06 \backslash \mathrm{Dipole}=0.0443419,-0.1$ $908163,0.7726805 \backslash \mathrm{PG}=\mathrm{C} 01 \quad[\mathrm{X}(\mathrm{C} 4 \mathrm{H} 13 \mathrm{P} 1 \mathrm{~S} 2)] \backslash \backslash \mathrm{a}$

\section{$\mathrm{S}=\mathrm{P}\left(\mathrm{CH}_{3}\right)\left(\mathrm{OCH}_{3}\right) \mathrm{SCH}_{3}$}

$1 \backslash 1 \backslash G I N C-S C 42 \backslash F O p t \backslash R B 3 L Y P \backslash 6-31 G(d) \backslash C 3 H 901 P 1 S 2 \backslash J O H 501 \backslash 09-A u g-2004 \backslash 0 \backslash \backslash \#$ B3LYP/6-31G* OPT FREQ MAXDISK=268435456\\s=pmesmeoch3 2 opt freq b3lyp $\backslash \backslash 0,1 \backslash \mathrm{P},-0.0438258315,-0.4034596468,0.4042411222 \backslash \mathrm{S}, 1 . \overline{8} 837702795,-0.145$ $0786206,0.6071416908 \backslash \mathrm{S},-1.1538166991,1.3348278698,-0.1801427958 \backslash \mathrm{C}, 0.15$ $43949698,2.5092496843,-0.7087449028 \backslash C,-0.9605336748,-0.9890015028,1.86$ $67483064 \backslash 0,-0.5142133746,-1.5797724061,-0.6320320508 \backslash \mathrm{C},-0.0205077954,-$ $1.5939146014,-1.9799858793 \backslash \mathrm{H},-0.5832441801,-1.9793883928,2.1353025856 \backslash$ $\mathrm{H},-2.0290895672,-1.0533516679,1.640990505 \backslash \mathrm{H},-0.7961666693,-0.294751510$ $6,2.6932305102 \backslash \mathrm{H}, 0.4936274211,2.290961103,-1.7226762551 \backslash \mathrm{H}, 0.9988257057$ , $2.4689363702,-0.018184394 \backslash \mathrm{H},-0.306616841,3.4995577646,-0.679008662 \backslash \mathrm{H}$, 
$-0.4048626038,-2.5089474875,-2.4336993609 \backslash \mathrm{H},-0.3943611941,-0.724961941$ $6,-2.5335459841 \backslash \mathrm{H}, 1.0736041139,-1.6019697545,-1.9898568358 \backslash \backslash$ Version $=\mathrm{DE}$ C-AXP-OSF $/ 1-G 03 R e v B .03 \backslash$ State $=1-A \backslash H F=-1332.7465946 \backslash$ RMSD $=8.198 \mathrm{e}-09 \backslash \mathrm{RMSF}=$ $5.513 e-06 \backslash \mathrm{Dipole}=-0.7023469,0.0508038,-0.4582846 \backslash \mathrm{PG}=\mathrm{C} 01$ [X(C3H901P1S2) ]$\backslash \backslash @$

\section{TS: $\bullet \mathrm{CH}_{3}+\mathrm{S}=\mathrm{P}\left(\mathrm{CH}_{3}\right)\left(\mathrm{OCH}_{3}\right) \mathrm{SCH}_{3}$}

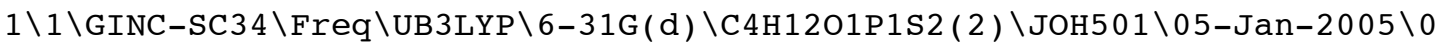
$\backslash \backslash \#$ B3LYP/6-31G* OPT=( TS , CALCALL, NOEIGENTEST, MAXCYC=200) MAXDISK=13421 $7728 \backslash \backslash$ ts ch3spmesmeoch3 up1 transition state search $\backslash \backslash 0,2 \backslash S,-1.65410537$ $81,-0.29 \overline{9} 6718383,0.1809 \overline{2} 219 \backslash P, 0.3799175276,-0.2772880811,0.1714742548 \backslash$ $\mathrm{S}, 1.4789133257,1.5988992836,0.416367051 \backslash \mathrm{C}, 0.2169125703,2.8158279742,-0$ $.1363018764 \backslash \mathrm{C}, 1.1074218807,-1.2766151137,1.514679707 \backslash 0,1.0790311648,-1$ $.0785403829,-1.0837888779 \backslash \mathrm{H}, 0.4248849741,3.7463297362,0.3968202919 \backslash \mathrm{H}, 0$ $.2834479883,2.9894796988,-1.2123715915 \backslash \mathrm{H},-0.7851920348,2.4633708417,0$. $1227076531 \backslash \mathrm{H}, 0.7875046509,-2.3145502403,1.3905229015 \backslash \mathrm{H}, 0.7564641249,-0$ $.8945972015,2.4755235068 \backslash \mathrm{H}, 2.1992891837,-1.2244862556,1.4714791021 \backslash \mathrm{C},-$ $3.3137557697,-1.794986842,0.7012425008 \backslash \mathrm{H},-4.1786827407,-1.1673853002,0$ $.4994667058 \backslash \mathrm{H},-3.1590984219,-2.6134300711,0.0047859564 \backslash \mathrm{H},-3.143018734$, $-2.0285866906,1.747743844 \backslash \mathrm{C}, 0.8202377024,-0.635144952,-2.4231088485 \backslash \mathrm{H}$, $1.3010184335,-1.3592992228,-3.0834502134 \backslash \mathrm{H},-0.2561447077,-0.6079918746$ $,-2.6218279681 \backslash \mathrm{H}, 1.2566895874,0.3566653355,-2.5888997411 \backslash \backslash$ Version=DEC$\mathrm{AXP}-\mathrm{OSF} / 1-\mathrm{G} 03 \mathrm{RevB} .03 \backslash \mathrm{State}=2-\mathrm{A} \backslash \mathrm{HF}=-1372.5726215 \backslash \mathrm{S} 2=0.765112 \backslash \mathrm{S} 2-1=0 . \backslash \mathrm{S} 2$ $\mathrm{A}=0.750076 \backslash \mathrm{RMSD}=8.869 \mathrm{e}-09 \backslash \mathrm{RMSF}=5.846 \mathrm{e}-06 \backslash \mathrm{Dipole}=0.2496645,-0.1325,-0.2$ $990281 \backslash$ DipoleDeriv $=0.1032463,0.6008492,-0.1284285,0.5176313,-0.0407083$ $,-0.1433561,-0.202369,-0.1232502,-0.4091402,1.8539205,0.2488989,-0.282$ $4049,-0.0555672,1.5373407,0.4235486,0.0719838,0.2827818,1.3693022,-0.9$ $063117,-0.6322394,0.057741,-0.567044,-0.940123,0.0347453,-0.1541907,-0$ $.1339652,-0.2320421,0.019523,-0.1774763,0.0559781,-0.0031522,0.2353551$ $,-0.0735221,-0.0025829,-0.135318,0.089593,-0.2489157,0.0393139,-0.0926$ $615,0.0752311,-0.256168,0.0900273,-0.0069147,0.0206025,-0.2258524,-0.7$ $507418,0.0990815,0.2962013,0.3679316,-0.5806413,-0.2872136,0.3326884$, $0.1695922,-1.6143668,0.0691614,0.0218468,-0.0277184,0.0101989,-0.10137$ $41,-0.0414391,-0.0265882,-0.0132225,0.0500542,0.0436969,0.0875777,-0.0$ $303504,0.0412352,-0.0101714,0.0463239,-0.0066834,0.0947665,-0.0931083$, $-0.0066573,0.077948,0.0005197,0.0150313,0.0913727,0.0265403,0.0120959$, $0.0737615,0.0527352,0.0609052,-0.022087,-0.0212234,-0.0201719,-0.04514$ $19,-0.003328,-0.0202856,0.0357779,0.0677582,0.0587152,0.0148941,0.0217$ $386,0.0050629,0.056384,-0.0174838,0.0355881,-0.0337166,-0.0114413,-0.0$ $591832,0.0035239,-0.0068046,0.0148299,0.0791746,0.0257901,-0.0422118,0$ $.0227324,0.0610861,-0.3169712,-0.2881027,0.0483432,-0.2885358,-0.16003$ $31,0.0664309,0.1072744,0.0843863,0.073776,-0.1009311,0.0157838,-0.0127$ $127,-0.0217226,-0.0210798,0.0189598,0.0075966,0.0246081,0.0470337,-0.0$ $22533,-0.0472953,0.0197305,-0.0420592,-0.0691221,-0.0522399,0.0320091$, $-0.0368702,-0.0021158,-0.0235891,-0.0464864,0.0055374,-0.0550805,-0.00$ $22801,0.0499285,0.0006042,0.0408766,-0.0698904,0.2146969,0.015494,0.13$ $98729,0.0339909,0.2573514,-0.2392506,-0.2145405,0.0393972,0.9338007,0$. $0411894,0.0547074,0.0585158,0.066989,-0.00446,-0.0793317,0.0589651,-0$. $059971,-0.0786899,-0.0663484,-0.0085741,-0.0914703,-0.0181553,0.053181$ $3,0.0548308,0.0185487,0.0025421,0.0077299,0.0371278,-0.0576579,-0.0104$ $039,-0.0766431,-0.0788565,0.1000394,-0.0009876,-0.0163269,-0.0162222 \backslash P$ olar $=155.8461992,39.8139532,129.7928069,-6.7196766,-5.340568,89.516374$ $4 \backslash \mathrm{PG}=\mathrm{C} 01 \quad[\mathrm{X}(\mathrm{C} 4 \mathrm{H} 12 \mathrm{O} 1 \mathrm{P} 1 \mathrm{~S} 2)] \backslash \mathrm{NImag}=1 \backslash \backslash$

\section{$\mathrm{CH}_{3} \mathrm{SP} \cdot\left(\mathrm{CH}_{3}\right)\left(\mathrm{OCH}_{3}\right) \mathrm{SCH}_{3}$}

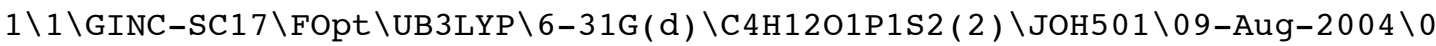
$\backslash \backslash \#$ B3LYP/6-31G* OPT FREQ MAXDISK=268435456\\ch3spmesmeoch3_3 opt freq b31yp $\backslash \backslash 0,2 \backslash P,-0.1491114504,0.397755971,0.2184787951 \backslash \mathrm{S}, 1.92 \overline{1} 3916583,1$. $0499705176,-0.5684791392 \backslash \mathrm{C},-0.7925406471,2.1004362881,0.469564828 \backslash 0,0$. $0665973067,0.0557547052,1.8267304905 \backslash \mathrm{C}, 2.3261717559,-0.3895271167,-1.6$ $345621622 \backslash \mathrm{H},-1.699601128,2.0634537041,1.0789586693 \backslash \mathrm{H},-1.0171014044,2.5$ $52605241,-0.4989028454 \backslash \mathrm{H},-0.0366862507,2.6985313866,0.9854687175 \backslash \mathrm{H}, 1.5$ $760809628,-0.5272812024,-2.4172873405 \backslash \mathrm{H}, 2.4311696207,-1.3107416182,-1$. 
$0558605941 \backslash \mathrm{H}, 3.2853005088,-0.1636265205,-2.1079624637 \backslash \mathrm{S},-2.173793033,-$ $0.4750776993,-0.422211953 \backslash \mathrm{C},-1.6461306885,-2.0668653051,-1.1708881542 \backslash$ $\mathrm{H},-2.3839225915,-2.3320147715,-1.9320641824 \backslash \mathrm{H},-1.6026232349,-2.8635695$ $731,-0.4241238584 \backslash \mathrm{H},-0.6698234804,-1.9641472899,-1.6528322555 \backslash \mathrm{C}, 0.6743$ $353648,-1.1875158705,2.1846475806 \backslash \mathrm{H}, 0.7347967014,-1.2007757663,3.27497$ $93371 \backslash \mathrm{H}, 0.0680586368,-2.0381350042,1.8496744609 \backslash \mathrm{H}, 1.6856522463,-1.2641$ $288589,1.7674114269 \backslash \backslash$ Version=DEC-AXP-OSF $/ 1-G 03$ RevB . 03 $\backslash$ State $=2-A \backslash H F=-13$ $72.5964691 \backslash \mathrm{S} 2=0.757646 \backslash \mathrm{S} 2-1=0 . \backslash \mathrm{S} 2 \mathrm{~A}=0.750034 \backslash \mathrm{RMSD}=6.517 \mathrm{e}-09 \backslash \mathrm{RMSF}=2.056 \mathrm{e}$ $-05 \backslash$ Dipole $=0.341014,-0.90723,-0.0409055 \backslash \mathrm{PG}=\mathrm{C} 01$ [X(C4H1201P1S2)] \\

\section{$\mathrm{CH}_{3} \mathrm{SP}(\mathrm{H})\left(\mathrm{CH}_{3}\right)\left(\mathrm{OCH}_{3}\right) \mathrm{SCH}_{3}$}

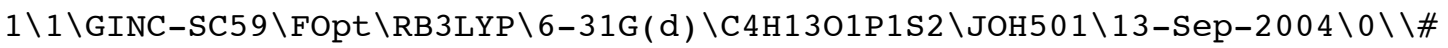
B3LYP/6-31G* OPT FREQ MAXDISK=134217728\\a2_ch3spmesmeoch3_3 opt freq b3lyp $\backslash \backslash 0,1 \backslash P,-0.1036034496,0.2952567759,0.2 \overline{8} 56549653 \backslash \mathrm{S}, 1.9 \overline{8} 57734777,0$ $.5626522369,-0.2768023945 \backslash \mathrm{C},-0.3260681852,2.1548262597,0.4285449649 \backslash 0$, $-0.5024825938,0.1513881465,1.9014091989 \backslash \mathrm{C}, 2.5562755688,-1.1190697922,-$ $0.7158231564 \backslash \mathrm{H},-1.3426741027,2.3672943854,0.7694264299 \backslash \mathrm{H},-0.1513023343$ $, 2.6280060658,-0.5403180183 \backslash \mathrm{H}, 0.3827775816,2.5433611541,1.164701039 \backslash \mathrm{H}$, $1.8616809547,-1.871896681,-0.3254207164 \backslash \mathrm{H}, 3.5377431668,-1.2828397943,-$ $0.2645297428 \backslash \mathrm{H}, 2.6300334577,-1.2278492837,-1.800242296 \backslash \mathrm{S},-1.6662777309$ $, 0.202074842,-1.2330741783 \backslash \mathrm{C},-1.6916065284,-1.5485428841,-1.7630773157$ $\backslash \mathrm{H},-1.3555726832,-1.6308797961,-2.7991173514 \backslash \mathrm{H},-2.708664915,-1.9375881$ $435,-1.6754100983 \backslash \mathrm{H},-1.0299959335,-2.1469651732,-1.1253443768 \backslash \mathrm{C},-0.514$ $4790501,-1.1246266984,2.5364495882 \backslash \mathrm{H},-0.9125655447,-0.9664342409,3.541$ $6916299 \backslash \mathrm{H},-1.1591607169,-1.8383538043,2.009138557 \backslash \mathrm{H}, 0.4963710677,-1.54$ $28547702,2.6157228045 \backslash \mathrm{H}, 0.0685797181,-1.1441113005,0.1750647482 \backslash \backslash$ Versi on=DEC-AXP-OSF $/ 1-G 03 R e v B .03 \backslash$ State $=1-A \backslash H F=-1373.1985877 \backslash$ RMSD $=7.214 e-09 \backslash$ $\mathrm{RMSF}=1.123 \mathrm{e}-04 \backslash \mathrm{Dipole}=0.0754443,-1.2533032,0.181132 \backslash \mathrm{PG}=\mathrm{C} 01$ [X $(\mathrm{C} 4 \mathrm{H} 1301 \mathrm{P}$ $1 \mathrm{~S} 2) \mathrm{J} \backslash \backslash @$

\section{$\mathrm{S}=\mathrm{P}\left(\mathrm{CH}_{3}\right)(\mathrm{CN}) \mathrm{SCH}_{3}$}

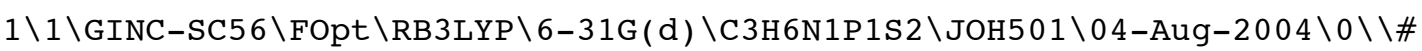
B3LYP/6-31G* OPT FREQ MAXDISK=268435456\\s=pmesmecn_1 optimisation b31 $\mathrm{yp} \backslash \backslash 0,1 \backslash \mathrm{S},-1.9869365043,-0.5313718746,-0.2588255516 \backslash \mathrm{P},-0.0390064606,-0$ $.4255487876,-0.2967876983 \backslash \mathrm{S}, 0.8703769925,1.4957886072,-0.3516690519 \backslash \mathrm{C}$, $0.7115030596,-1.2093268204,1.1457061162 \backslash \mathrm{C}, 0.8382701621,-1.2677637073,-$ $1.6649490288 \backslash \mathrm{C},-0.1621247135,2.395339362,0.8730158262 \backslash \mathrm{H}, 0.5815946179,-$ $2.3300066762,-1.6349485918 \backslash \mathrm{H}, 1.9208906027,-1.1437428262,-1.5651079476 \backslash$ $\mathrm{H}, 0.4947230355,-0.8372121087,-2.6092309246 \backslash \mathrm{H}, 0.0901581968,2.093594915$, $1.8912893169 \backslash \mathrm{H},-1.2207246235,2.2192903439,0.6696869771 \backslash \mathrm{H}, 0.0694661465$, $3.4536096772,0.7334015906 \backslash \mathrm{N}, 1.1697214389,-1.716066034,2.087428747 \backslash \backslash \mathrm{Ver}$ sion=DEC-AXP-OSF $/ 1-G 03$ RevB .03 \State $=1-A \backslash H F=-1310.426487 \backslash$ RMSD $=7.897 e-09$ $\backslash \mathrm{RMSF}=5.634 \mathrm{e}-06 \backslash \mathrm{Dipole}=0.5694214,0.7486064,-0.8826935 \backslash \mathrm{PG}=\mathrm{C} 01 \quad[\mathrm{X}(\mathrm{C} 3 \mathrm{H} 6 \mathrm{~N} 1$ $\mathrm{P} 1 \mathrm{~S} 2$ ) $] \backslash \backslash @$

\section{TS: $\bullet \mathrm{CH}_{3}+\mathrm{S}=\mathrm{P}\left(\mathrm{CH}_{3}\right)(\mathrm{CN}) \mathrm{SCH}_{3}$}

$1 \backslash 1 \backslash G I N C-S C 64 \backslash$ Freq $\backslash U B 3 L Y P \backslash 6-31 G(d) \backslash C 4 H 9 N 1 P 1 S 2(2) \backslash J O H 501 \backslash 06-J a n-2005 \backslash 0 \backslash$ $\backslash \#$ B3LYP /6-31G* OPT $=($ TS , CALCALL , NOEIGENTEST , MAXCYC $=200)$ MAXDISK=134217 728\\ts_ch3spmesmecn_up1 transition state search $\backslash \backslash 0,2 \backslash S,-1.6932381919$, $-0.3211 \overline{2} 0612,0.12199 \overline{7} 2433 \backslash \mathrm{P}, 0.3174785271,-0.2244319847,0.1353738353 \backslash \mathrm{S}$, $1.447446849,1.5043516293,0.7909605747 \backslash \mathrm{C}, 0.5023033372,2.8217303076,-0.0$ $740977884 \backslash \mathrm{C}, 1.1232069843,-1.520591495,1.1528032148 \backslash \mathrm{C}, 0.9863933752,-0.5$ $823745593,-1.4999377177 \backslash \mathrm{H}, 0.8259872711,3.7659107079,0.3697038469 \backslash \mathrm{H}, 0.7$ $261338179,2.8245116124,-1.142754045 \backslash \mathrm{H},-0.569904444,2.6907152267,0.0907$ $541703 \backslash \mathrm{H}, 0.7842495044,-2.5000260945,0.806064167 \backslash \mathrm{H}, 0.8175968655,-1.3763$ $819593,2.19261981 \backslash \mathrm{H}, 2.2119936041,-1.4549328852,1.0696339635 \backslash \mathrm{C},-3.38805$ $28522,-2.0016793622,0.0639210686 \backslash \mathrm{H},-4.237445327,-1.3254454694,0.033448$ $0717 \backslash \mathrm{H},-3.1535604679,-2.5290042247,-0.8541909796 \backslash \mathrm{H},-3.2244985409,-2.53$ $48372895,0.9941465866 \backslash \mathrm{N}, 1.3781180328,-0.7754622113,-2.5790701247 \backslash \backslash$ Vers ion=DEC-AXP-OSF $/ 1-G 03 R e v B .03 \backslash$ State $=2-A \backslash H F=-1350.2569503 \backslash \mathrm{S} 2=0.7672 \backslash \mathrm{S} 2-1$ $=0 . \backslash \mathrm{S} 2 \mathrm{~A}=0.750091 \backslash \mathrm{RMSD}=6.509 \mathrm{e}-09 \backslash \mathrm{RMSF}=1.024 \mathrm{e}-06 \backslash \mathrm{Dipole}=-0.0042751,-0.02$ $44774,1.0345961 \backslash$ DipoleDeriv $=0.1100228,0.554998,-0.0172675,0.5291091,0$. 
$0290273,-0.0069431,0.0329346,0.0183946,-0.4178663,1.6459611,0.2947699$, $0.0224906,0.1475675,1.2747124,0.1633776,0.2048021,0.3425624,1.1018939$, $-0.9071362,-0.6368568,-0.0911059,-0.5191729,-0.804111,-0.0847143,-0.28$ $33837,-0.2707337,-0.2499765,0.0422708,-0.1278245,0.0380042,0.0219572,0$ $.2180707,-0.0823938,-0.0292662,-0.1929378,0.0858296,-0.2259406,0.06655$ $53,-0.0541169,0.040588,-0.2890825,0.0482329,0.0056797,-0.0611208,-0.18$ $66101,-0.2460732,0.0017125,0.1765198,0.1989996,-0.1033777,-0.1159433,-$ $0.0393821,-0.06312,-0.391703,0.0837434,-0.0010766,-0.0338259,-0.012695$ $3,-0.0690275,-0.0210043,-0.0284476,0.0113355,0.0639857,0.0636163,0.080$ $3724,-0.0069088,0.0335218,0.0122179,0.0258472,0.0120979,0.0801055,-0.0$ $696476,-0.0158333,0.0746744,-0.0008381,0.0057644,0.0668413,0.0359621,0$ $.0173478,0.1007316,0.0527784,0.0577195,-0.0125097,-0.0257456,-0.019423$ $2,-0.0216181,-0.0222515,-0.0233205,0.0170708,0.0707607,0.0666454,0.010$ $8453,0.0070643,-0.0044084,0.0801301,0.0072974,0.0199282,-0.0240514,-0$. $0065572,-0.0371819,0.0039527,0.0000683,0.0225449,0.0684983,0.0239996,-$ $0.0327242,0.0345661,0.072361,-0.3497536,-0.2848535,0.0017056,-0.338751$ $5,-0.1908234,0.0011667,-0.0169245,-0.01198,0.0914974,-0.058206,0.03056$ $06,-0.0023371,-0.0185569,-0.0133561,0.0016663,-0.0033647,0.0014203,0.0$ $540799,-0.0068225,-0.03338,0.0116231,-0.0294439,-0.0102243,-0.0494268$, $0.0291376,-0.0326688,-0.0179928,-0.004106,-0.0340372,-0.0066951,-0.034$ $9018,-0.016609,0.0549368,-0.0238303,0.0388147,-0.0270009,-0.2189262,0$. $0120974,-0.0186351,-0.0226984,-0.2312682,0.0201905,0.158716,0.0116109$, $-0.2258322 \backslash$ Polar $=161.7874268,43.391201,128.413179,-1.1909354,5.2599973$ , $92.5953313 \backslash \mathrm{PG}=\mathrm{C} 01 \quad[\mathrm{X}(\mathrm{C} 4 \mathrm{H} 9 \mathrm{~N} 1 \mathrm{P} 1 \mathrm{~S} 2)] \backslash \mathrm{NImag}=1 \backslash \backslash$

\section{$\mathrm{CH}_{3} \mathrm{SP} \cdot\left(\mathrm{CH}_{3}\right)(\mathrm{CN}) \mathrm{SCH}_{3}$}

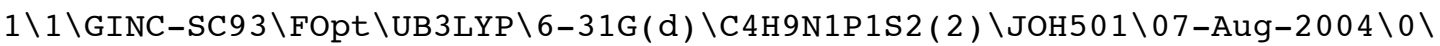
$\backslash \#$ B3LYP/6-31G* OPT FREQ MAXDISK=268435456\\ch3spmesmecn_2 opt freq $\backslash \backslash 0$ $, 2 \backslash \mathrm{P},-0.0479895821,0.2454296457,0.069709227 \backslash \mathrm{S}, 2.25799999 \overline{9} 4,0.102661957$ $4,0.2732965179 \backslash C,-0.3556409791,2.0321315783,0.473416093 \backslash \mathrm{C},-0.326285584$ $8,-0.5434290553,1.6577944787 \backslash \mathrm{C}, 2.7023781392,0.7258233,-1.3949264507 \backslash \mathrm{H}$, $-1.3642148178,2.169083256,0.8704296934 \backslash \mathrm{H},-0.2501043885,2.6125531647,-0$ $.4481096159 \backslash \mathrm{H}, 0.3839814825,2.3687344474,1.2017968664 \backslash \mathrm{H}, 2.4655954545,1$. $786490719,-1.5126799453 \backslash \mathrm{H}, 2.2064482927,0.1442103732,-2.1765955999 \backslash \mathrm{H}, 3$. $7826198312,0.5937966825,-1.4973127684 \backslash \mathrm{S},-2.0957337616,-0.1212133564,-0$ $.8806390996 \backslash \mathrm{C},-1.9590710539,-1.9283486555,-1.1662623038 \backslash \mathrm{H},-2.881242968$ $1,-2.2339558996,-1.6668479268 \backslash \mathrm{H},-1.8705783896,-2.4690555659,-0.2208023$ $781 \backslash \mathrm{H},-1.1115901826,-2.1619903912,-1.8152947078 \backslash \mathrm{N},-0.5150876451,-1.130$ $2217276,2.6453040544 \backslash \backslash$ Version=DEC-AXP-OSF $/ 1-G 03$ RevB . 03 $\backslash$ State $=2-A \backslash H F=-1$ $350.2893525 \backslash \mathrm{S} 2=0.758832 \backslash \mathrm{S} 2-1=0 . \backslash \mathrm{S} 2 \mathrm{~A}=0.750038 \backslash \mathrm{RMSD}=6.506 \mathrm{e}-09 \backslash \mathrm{RMSF}=4.307$ e-05 $\backslash$ Dipole $=0.181267,0.7475778,-1.3833462 \backslash \mathrm{PG}=\mathrm{C} 01$ [X(C4H9N1P1S2)] $\backslash \backslash$

\section{$\mathrm{CH} 3 \mathrm{SP}(\mathrm{H})(\mathrm{CH} 3)(\mathrm{CN}) \mathrm{SCH} 3$}

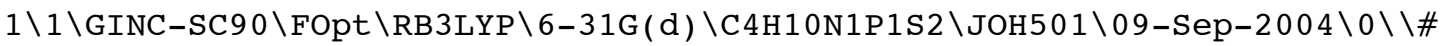
B3LYP/6-31G* OPT FREQ MAXDISK=134217728\\a_ch3spmesmecn_2 opt freq $\backslash \backslash 0$ $, 1 \backslash P,-0.0357257815,0.1696801305,0.214979237 \overline{2} \backslash \mathrm{S}, 1.6967130 \overline{6} 6,1.003599275$ $1,-0.821869205 \backslash \mathrm{C},-0.59379669,1.9169682168,0.6674466116 \backslash \mathrm{C}, 0.3157841807$, $-0.306654397,1.9467786457 \backslash \mathrm{C}, 2.5875334067,-0.4634738063,-1.4479623397 \backslash \mathrm{H}$ $,-1.4494642205,1.8551840732,1.345973585 \backslash \mathrm{H},-0.8790007078,2.4588816054,-$ $0.2385415677 \backslash \mathrm{H}, 0.2286296928,2.4377995974,1.1661934059 \backslash \mathrm{H}, 1.9577640238$,$1.3574567741,-1.3563017059 \backslash \mathrm{H}, 3.5060270602,-0.6183838664,-0.8783248293 \backslash$ $\mathrm{H}, 2.8249157062,-0.3066722963,-2.5023710999 \backslash \mathrm{S},-2.0725469742,-0.30435303$ $54,-0.416942945 \backslash \mathrm{C},-1.9750314894,-2.0465316347,-0.9583077816 \backslash \mathrm{H},-2.45255$ $97499,-2.1377321663,-1.936247555 \backslash \mathrm{H},-2.4769928867,-2.6942114032,-0.2367$ $367296 \backslash \mathrm{H},-0.9265754825,-2.3583992015,-1.0461604905 \backslash \mathrm{N}, 0.5480500245,-0.6$ $379594359,3.0375700013 \backslash \mathrm{H}, 0.3731991983,-1.1482855837,-0.2318979967 \backslash \backslash$ Ver sion=DEC-AXP-OSF /1-G03RevB.03 \State $=1-A \backslash H F=-1350.8808875 \backslash$ RMSD $=5.030 e-0$ $9 \backslash \mathrm{RMSF}=1.022 \mathrm{e}-05 \backslash \mathrm{Dipole}=0.0081504,-0.4270293,-1.3104231 \backslash \mathrm{PG}=\mathrm{C} 01$ [X $(\mathrm{C} 4 \mathrm{H} 1$ $0 \mathrm{~N} 1 \mathrm{P} 1 \mathrm{~S} 2$ ) $] \backslash \backslash @$

\section{$\mathrm{S}=\mathrm{P}\left(\mathrm{CH}_{3}\right)\left(\mathrm{C}_{6} \mathrm{H}_{5}\right) \mathrm{SCH}_{3}$}

$1 \backslash 1 \backslash G I N C-S C 160 \backslash F O p t \backslash R B 3 L Y P \backslash 6-31 G(d) \backslash C 8 H 11 P 1 S 2 \backslash J O H 501 \backslash 07-A u g-2004 \backslash 0 \backslash \backslash \#$ 
B3LYP/6-31G* OPT FREQ MAXDISK=268435456 MAXDISK=268435456 MAXDISK=2684 $35456 \backslash \backslash \mathrm{s}=$ pmesmeph_1 optimisation b3lyp $\backslash \backslash 0,1 \backslash S,-2.4004752549,0.13118915$ $21,-0.9486812097 \backslash \overline{\mathrm{P}},-0.4316435533,0.1367766035,-0.9542154483 \backslash \mathrm{S}, 0.506938$ $2632,2.0621870438,-1.0848637438 \backslash \mathrm{C}, 0.3245246008,-0.7088546947,0.4964257$ $388 \backslash \mathrm{C}, 0.3406391765,-0.6617540683,-2.4188335557 \backslash \mathrm{C},-0.4277666789,2.96721$ $29529,0.2096286945 \backslash \mathrm{H}, 0.0403333927,-1.7131849972,-2.4285352226 \backslash \mathrm{H}, 1.4321$ $745116,-0.5947644745,-2.3776644525 \backslash \mathrm{H},-0.0274958728,-0.1728807195,-3.32$ $41209962 \backslash \mathrm{H},-0.1532535281,2.6197563857,1.2079954815 \backslash \mathrm{H},-1.5007378882,2.8$ $430319298,0.0454181602 \backslash \mathrm{H},-0.1563198517,4.0196444994,0.0997549111 \backslash \mathrm{C},-0$. $4890969667,-1.4242795037,1.3832632468 \backslash \mathrm{C}, 0.0801360193,-2.0999311371,2.4$ $643899131 \backslash \mathrm{C}, 1.4601240263,-2.0646601405,2.6659565084 \backslash \mathrm{C}, 1.7119096471,-0$. $6692317604,0.7067902021 \backslash \mathrm{C}, 2.2754569196,-1.3479562617,1.7860637994 \backslash \mathrm{H},-1$ $.5624422616,-1.4433941084,1.220733639 \backslash \mathrm{H},-0.5579497103,-2.6514605089,3$. $1494051833 \backslash \mathrm{H}, 1.9011976049,-2.5899552901,3.508856915 \backslash \mathrm{H}, 2.3502395253,-0$. $0938139123,0.0421519098 \backslash \mathrm{H}, 3.3499387795,-1.3119193073,1.9438481673 \backslash \backslash$ Ver sion=DEC-AXP-OSF /1-G03RevB.03\State $=1-A \backslash H F=-1449.2513267 \backslash$ RMSD $=6.144 \mathrm{e}-0$ $9 \backslash \mathrm{RMSF}=2.696 \mathrm{e}-06 \backslash \mathrm{Dipole}=1.4088887,-0.3427204,0.6662138 \backslash \mathrm{PG}=\mathrm{C} 01 \quad[\mathrm{X}(\mathrm{C} 8 \mathrm{H} 11$ P1S2 ) $] \backslash \backslash @$

\section{TS: $\cdot \mathrm{CH}_{3}+\mathrm{S}=\mathrm{P}\left(\mathrm{CH}_{3}\right)\left(\mathrm{C}_{6} \mathrm{H}_{5}\right) \mathrm{SCH}_{3}$}

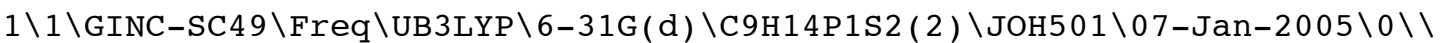
\# B3LYP $/ 6-31 \mathrm{G} * \mathrm{OPT}=(\mathrm{TS}, \mathrm{CALCALL}, \mathrm{NOEIGENTEST}, \mathrm{MAXCYC}=200)$ MAXDISK $=2684354$ 56\\ts_ch3spmesmeph_up1 transition state search $\backslash \backslash 0,2 \backslash S,-2.1773105383,0$ $.37089 \overline{2} 0466,0.60145 \overline{6} 2673 \backslash \mathrm{P},-0.1419706587,0.329856165,0.6845244468 \backslash \mathrm{S}, 1$. $0833241178,2.1506978309,0.5195165358 \backslash \mathrm{C}, 0.1030300695,3.2634303857,1.604$ $3779667 \backslash \mathrm{C}, 0.415642977,-0.4591780524,2.2563330284 \backslash \mathrm{C}, 0.6248662704,-0.672$ $302709,-0.6471867356 \backslash \mathrm{H}, 0.432603428,4.2807859981,1.3802001133 \backslash \mathrm{H},-0.9622$ $672741,3.1713257362,1.3757093521 \backslash \mathrm{H}, 0.2783346954,3.0540449751,2.6624980$ $882 \backslash \mathrm{H},-0.0263842821,-1.4569764295,2.3300643812 \backslash \mathrm{H}, 0.0636430836,0.146788$ $8565,3.0957530944 \backslash \mathrm{H}, 1.5054879369,-0.531921374,2.2962022217 \backslash \mathrm{C},-3.876479$ $3991,-1.2201094858,0.7015559655 \backslash \mathrm{H},-4.7131614329,-0.5724915707,0.451807$ $5311 \backslash \mathrm{H},-3.6051979607,-1.9489203963,-0.05598421 \backslash \mathrm{H},-3.8423952697,-1.5696$ $290321,1.7286269337 \backslash \mathrm{C},-0.1515073309,-1.1037923964,-1.7301344945 \backslash \mathrm{C}, 0.43$ $09439503,-1.846780568,-2.7571972286 \backslash C, 1.7903655836,-2.1605710597,-2.71$ $37816391 \backslash \mathrm{C}, 1.9929180592,-0.9864038982,-0.6094065626 \backslash \mathrm{C}, 2.5706917678,-1$. $7277996122,-1.6399526886 \backslash \mathrm{H},-1.2076353522,-0.8538165377,-1.7593198706 \backslash \mathrm{H}$ $,-0.1789754111,-2.178605235,-3.5929718228 \backslash \mathrm{H}, 2.2418902079,-2.7392989911$ $,-3.5150824328 \backslash \mathrm{H}, 2.6143637607,-0.6476094151,0.2145544503 \backslash \mathrm{H}, 3.630204792$ $3,-1.9659127222,-1.6031350503 \backslash \backslash$ Version=DEC-AXP-OSF /1-G03RevB.03 $\backslash$ State= $2-\mathrm{A} \backslash \mathrm{HF}=-1489.077371 \backslash \mathrm{S} 2=0.766448 \backslash \mathrm{S} 2-1=0 . \backslash \mathrm{S} 2 \mathrm{~A}=0.750084 \backslash \mathrm{RMSD}=9.327 \mathrm{e}-09 \backslash \mathrm{RM}$ $\mathrm{SF}=2.181 \mathrm{e}-06 \backslash \mathrm{Dipole}=0.7815697,-0.7211173,0.2694006 \backslash \mathrm{DipoleDeriv}=0.10375$ $27,0.5172837,-0.0208432,0.5857236,-0.0899488,-0.1017696,0.0182742,-0.0$ $71444,-0.4767036,1.7088449,0.2150739,-0.171914,0.086072,1.4073093,0.20$ $62679,-0.2163056,0.1174491,1.0825035,-1.0667461,-0.5886591,0.0732292,-$ $0.5914368,-0.8026934,-0.0062211,0.0325675,0.0350109,-0.2708037,0.05284$ $18,-0.1355148,-0.0993581,0.0388801,0.1159273,0.0469955,0.0572795,0.198$ $6984,0.1830894,-0.2261768,0.0252033,-0.0496421,0.1494071,-0.2637305,0$. $0191101,0.0232399,-0.0250563,-0.29072,-0.2757559,0.0703202,0.0823127,0$ $.0327088,-0.245973,-0.0591968,-0.0681798,-0.0189416,-0.246911,0.076862$ $1,-0.0084641,0.0385256,-0.0151947,-0.101998,-0.0259705,0.0266992,-0.05$ $51417,0.0378812,-0.0074909,0.0844451,0.015176,0.008624,0.1023328,-0.01$ $63613,-0.0179059,-0.0834899,0.0183937,0.0507333,0.0768955,0.037263,0.0$ $304337,0.0120523,-0.0043297,-0.0044457,-0.0729134,-0.1455938,0.0259459$ $,-0.0424539,-0.0152177,-0.0257613,-0.043402,0.0220004,-0.0361037,0.067$ $0473,0.0605632,0.0663802,0.0190932,0.0141072,0.0014507,0.0487706,-0.04$ $10224,0.0554991,-0.0744292,-0.0455904,-0.0789474,0.0253003,-0.0172907$, $0.0358845,0.0675016,0.0367237,-0.05401,0.0356275,0.0610966,-0.3523999$, $-0.2448175,0.0147545,-0.318639,-0.1418516,0.0166738,0.0109979,0.022858$ $8,0.0921381,-0.1017708,0.024998,-0.0191203,-0.0265974,-0.0157557,0.018$ $612,-0.0117434,0.0182213,0.043922,-0.0176544,-0.0290767,0.0200172,-0.0$ $281848,-0.0559467,-0.0572963,0.0214031,-0.0542994,-0.0155713,-0.029231$ $4,-0.0484837,0.0119845,-0.0618424,-0.012459,0.0565414,0.0031302,0.0401$ $789,-0.0590977,-0.0539678,0.0695442,0.1630176,-0.0186051,-0.0765638,-0$ 
$.0589803,-0.0541053,0.0315103,-0.0748496,-0.0087998,0.0518275,0.089271$ $7,0.0495333,-0.0630146,0.0757813,0.136648,0.046817,0.0683073,-0.029007$ $3,-0.0390667,-0.0089066,-0.0374851,-0.0246049,0.0834195,-0.0494152,0.0$ $91654,0.0602515,0.0202603,-0.0737181,-0.0674723,0.0854854,-0.0859565,0$ $.0448754,0.1757955,-0.0218702,-0.0393158,0.139737,-0.0409376,0.0213615$ $,-0.0485605,-0.0948758,-0.0307731,0.0238214,-0.0169088,-0.1453299,-0.0$ $014697,0.0034953,-0.0308275,0.0085508,0.092542,0.0170373,-0.0119678,0$. $0260967,0.128678,0.0112452,-0.0196601,-0.0759015,-0.0114714,0.0740499$, $-0.0705769,-0.0504674,-0.0728607,-0.0452961,0.0364909,0.0499254,0.0552$ $735,0.0533894,0.0394703,-0.0941051,0.057441,-0.0903251,-0.0243485,0.06$

$478,180.0222758,-0.0682795,-0.0341703,0.0803449,-0.0478409,-0.0688352,-$ $0.0439137,-0.0152942,-0.108458,0.0597225,0.0084793,0.0518054,0.07847$

$35,-0.0295944,0.0006886,-0.0295761,0.0586009 \backslash$ Polar $=218.0577918,22.9219$ $415,158.5464539,-13.7565922,37.8763888,144.2712208 \backslash \mathrm{PG}=\mathrm{C} 01 \quad[\mathrm{X}(\mathrm{C} 9 \mathrm{H} 14 \mathrm{P} 1 \mathrm{~S} 2$ ) $] \backslash N \operatorname{mag}=1 \backslash \backslash$

\section{$\mathrm{CH}_{3} \mathrm{SP} \cdot\left(\mathrm{CH}_{3}\right)\left(\mathrm{C}_{6} \mathrm{H}_{5}\right) \mathrm{SCH}_{3}$}

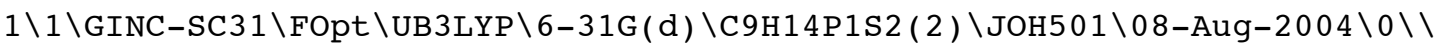
\# B3LYP/6-31G* OPT FREQ MAXDISK=268435456\\ch3spmesmeph_2 opt freq $\backslash \backslash 0$, $2 \backslash P, 0.0233613297,0.2806160263,-0.7000208959 \backslash S, 2.3217165 \overline{6} 39,0.532243285$ $8,-0.5956493788 \backslash \mathrm{C},-0.5399451247,2.0458933993,-0.8591534537 \backslash \mathrm{C},-0.175937$ $2282,-0.1186435646,1.0848203706 \backslash \mathrm{C}, 2.6849246941,0.7796399373,-2.3778979$ $626 \backslash \mathrm{H},-1.5336187554,2.1628631378,-0.4196139907 \backslash \mathrm{H},-0.5989132032,2.29525$ $43317,-1.9228848396 \backslash \mathrm{H}, 0.1724356687,2.7233407609,-0.3822642905 \backslash \mathrm{H}, 2.3075$ $179936,1.7386309172,-2.7440315142 \backslash \mathrm{H}, 2.2686207837,-0.0337068613,-2.9787$ $456624 \backslash \mathrm{H}, 3.7729217853,0.7678515017,-2.4845434531 \backslash \mathrm{S},-1.897681584,-0.817$ $1188431,-1.3905532482 \backslash \mathrm{C},-1.6478397008,-0.7216401413,-3.2059705624 \backslash \mathrm{H},-2$ $.4228729813,-1.3412677038,-3.664888037 \backslash \mathrm{H},-0.6699068786,-1.1206182235,-$ $3.4888980529 \backslash \mathrm{H},-1.7510564264,0.3006755734,-3.5810823238 \backslash \mathrm{C},-0.218779787$ $6,-1.4671766734,1.4725470991 \backslash \mathrm{C},-0.3259225843,-1.810237183,2.8196843512$ $\backslash C,-0.3871543485,-0.8130396008,3.7949100651 \backslash C,-0.2321472341,0.87670001$ $31,2.0707353912 \backslash \mathrm{C},-0.3392120899,0.529468333,3.4183395024 \backslash \mathrm{H},-0.17903326$ $72,-2.2472464538,0.7182522336 \backslash \mathrm{H},-0.3620297659,-2.8575857176,3.10654507$ $67 \backslash \mathrm{H},-0.470936057,-1.0815582884,4.8445626205 \backslash \mathrm{H},-0.1915283595,1.9259394$ $684,1.7964315211 \backslash \mathrm{H},-0.3844997363,1.310408961,4.1726273793 \backslash \backslash$ Version=DEC $-\mathrm{AXP}-\mathrm{OSF} / 1-\mathrm{G} 03 \mathrm{RevB} .03 \backslash \mathrm{State}=2-\mathrm{A} \backslash \mathrm{HF}=-1489.1050869 \backslash \mathrm{S} 2=0.757907 \backslash \mathrm{S} 2-1=0 . \backslash \mathrm{S}$ $2 \mathrm{~A}=0.750034 \backslash \mathrm{RMSD}=8.260 \mathrm{e}-09 \backslash \mathrm{RMSF}=4.001 \mathrm{e}-06 \backslash \mathrm{Dipole}=-0.11228,0.5552008,-0$ $.2732019 \backslash \mathrm{PG}=\mathrm{C} 01 \quad \mathrm{X}(\mathrm{C} 9 \mathrm{H} 14 \mathrm{P} 1 \mathrm{~S} 2)] \backslash \backslash @$

\section{$\mathrm{CH}_{3} \mathrm{SP}(\mathrm{H})\left(\mathrm{CH}_{3}\right)\left(\mathrm{C}_{6} \mathrm{H}_{5}\right) \mathrm{SCH}_{3}$}

$1 \backslash 1 \backslash G I N C-S C 88 \backslash F O p t \backslash R B 3 L Y P \backslash 6-31 G(d) \backslash C 9 H 15 P 1 S 2 \backslash J O H 501 \backslash 10-$ Sep-2004 $0 \backslash \backslash \# B$ $3 L Y P / 6-31 G *$ OPT FREQ MAXDISK $=268435456 \backslash \backslash$ a_ch3spmesmeph_2 opt freq $\backslash \backslash 0,1$ $\backslash P,-0.0577780481,0.2172055267,-0.78705217 \overline{7} \backslash \mathrm{S}, 1.9847172 \overline{7} 67,-0.038164665$ $6,-1.6350167494 \backslash \mathrm{C}, 0.0345224861,2.1005472439,-0.7039658332 \backslash \mathrm{C},-0.0411786$ $826,-0.2700410508,0.988987868 \backslash \mathrm{C}, 3.091812361,1.3720027325,-1.2324925285$ $\backslash \mathrm{H},-0.8854688248,2.5135455729,-0.280726115 \backslash \mathrm{H}, 0.1540406174,2.4783533592$ $,-1.7221898946 \backslash \mathrm{H}, 0.889639053,2.4120027425,-0.099078668 \backslash \mathrm{H}, 3.167208585,1$ $.5281633102,-0.1531644638 \backslash \mathrm{H}, 2.7735304097,2.2941542483,-1.7235918659 \backslash \mathrm{H}$, $4.0805906906,1.0986898859,-1.6123266591 \backslash \mathrm{S},-2.1557205332,0.4499354805,-$ $1.5217718782 \backslash \mathrm{C},-2.645538485,-1.2710667851,-1.8759226534 \backslash \mathrm{H},-3.287432541$ $8,-1.2736764844,-2.760156317 \backslash \mathrm{H},-3.1821987125,-1.7118096694,-1.03225978$ $02 \backslash \mathrm{H},-1.756647291,-1.8797617931,-2.0905515075 \backslash \mathrm{C}, 0.2076213271,-1.612832$ $9818,1.3084807597 \backslash \mathrm{C}, 0.2251652791,-2.0366119468,2.6362221272 \backslash \mathrm{C},-0.01254$ $69321,-1.1231268335,3.665162342 \backslash \mathrm{C},-0.2785558834,0.6398733987,2.0289660$ $765 \backslash \mathrm{C},-0.2657662576,0.2142098223,3.3580778553 \backslash \mathrm{H}, 0.4016658148,-2.327828$ $8046,0.5132581379 \backslash \mathrm{H}, 0.4247449932,-3.0795980797,2.8667933501 \backslash \mathrm{H},-0.00139$ $98884,-1.4523461076,4.700703855 \backslash \mathrm{H},-0.4871358541,1.6819848024,1.8127007$ $482 \backslash \mathrm{H},-0.4557408465,0.9302040795,4.1531616761 \backslash \mathrm{H},-0.1258846535,-1.13621$ $45966,-1.2992678822 \backslash \backslash$ Version=DEC-AXP-OSF $/ 1-G 03$ RevB . 03 $\backslash$ State $=1-A \backslash H F=-14$ $89.6942202 \backslash \mathrm{RMSD}=8.613 \mathrm{e}-09 \backslash \mathrm{RMSF}=2.299 \mathrm{e}-06 \backslash \mathrm{Dipole}=0.2274275,0.1058228,1$. $0052061 \backslash \mathrm{PG}=\mathrm{C} 01 \quad[\mathrm{X}(\mathrm{C} 9 \mathrm{H} 15 \mathrm{P} 1 \mathrm{~S} 2)] \backslash \backslash @$ 


\section{$\mathrm{S}=\mathrm{P}\left(\mathrm{OCH}_{3}\right)_{2} \mathrm{SCH}_{3}$}

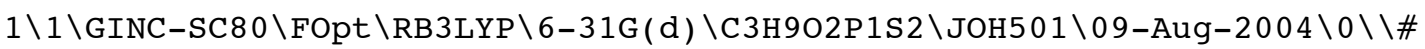
B3LYP/6-31G* OPT FREQ MAXDISK=268435456\\s=psch3och32_1 opt freq b3lyp $\backslash \backslash 0,1 \backslash S,-2.0706854079,-0.3616417522,-0.4703393835 \backslash \mathrm{P},-0.1560670956,-0.1$ $822679763,-0.222816478 \backslash \mathrm{S}, 0.6809939462,1.7544688982,-0.1381734802 \backslash 0,0.4$ $468854432,-0.8281971986,1.1518731873 \backslash 0,0.6904366303,-0.8686488178,-1.4$ $216148537 \backslash \mathrm{C},-0.3064989131,2.4607061896,1.2410623327 \backslash \mathrm{H},-0.0880304939,1$. $9405296153,2.1752539288 \backslash \mathrm{H},-1.3719115165,2.4025256575,1.0091510974 \backslash \mathrm{H},-0$ $.0040753655,3.5072852322,1.3214237435 \backslash \mathrm{C},-0.1141920219,-2.0432866318,1$. $691281064 \backslash \mathrm{H}, 0.4307101926,-2.2338342822,2.617504502 \backslash \mathrm{H},-1.1801153469,-1$. $914273076,1.8952156118 \backslash \mathrm{H}, 0.0271558958,-2.8778607346,0.9974451203 \backslash \mathrm{C}, 2.1$ $251365783,-1.0180960809,-1.3732590797 \backslash \mathrm{H}, 2.3695671328,-1.7858150064,-2$. $1093312429 \backslash \mathrm{H}, 2.6145453285,-0.0784458532,-1.645699293 \backslash \mathrm{H}, 2.4529735469,-1$ $.3324989755,-0.3790830506 \backslash \backslash$ Version=DEC-AXP-OSF / 1-G03RevB.03 \State $=1-A \backslash$ $\mathrm{HF}=-1407.9664634 \backslash \mathrm{RMSD}=5.606 \mathrm{e}-09 \backslash \mathrm{RMSF}=1.240 \mathrm{e}-05 \backslash \mathrm{Dipole}=1.2365744,-0.256$ $1119,0.9603489 \backslash \mathrm{PG}=\mathrm{C} 01 \quad[\mathrm{X}(\mathrm{C} 3 \mathrm{H} 902 \mathrm{P} 1 \mathrm{~S} 2)] \backslash \backslash @$

\section{TS: $\bullet \mathrm{CH}_{3}+\mathrm{S}=\mathrm{P}\left(\mathrm{OCH}_{3}\right)_{2} \mathrm{SCH}_{3}$}

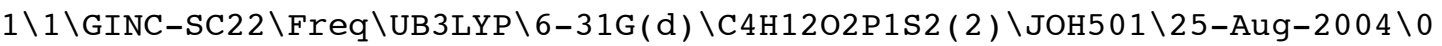
$\backslash \backslash \#$ B3LYP $/ 6-31 \mathrm{G}$ * OPT $=($ TS , CALCALL , NOEIGENTEST , MAXCYC $=200)$ MAXDISK $=26843$ 5456\\ts_ch3spsmeoch32_2 gaussian 98 transition state search $\backslash \backslash 0,2 \backslash S,-1$ $.7937609877,-0.4697569 \overline{8} 05,0.2286178263 \backslash \mathrm{P}, 0.1803462744,-0.0789987031,0$. $0638227513 \backslash \mathrm{S}, 0.9862849901,1.8788121879,-0.4620698536 \backslash \mathrm{C},-0.2787681298,2$ $.9514830546,0.3312791042 \backslash 0,0.9444622784,-0.5708051868,1.4031296263 \backslash 0,0$ $.9699547445,-0.9797552061,-1.0453620795 \backslash \mathrm{H},-0.1238385061,3.9551467454,-$ $0.071548002 \backslash \mathrm{H},-1.2836241376,2.6067097458,0.0770357551 \backslash \mathrm{H},-0.1543500245$, $2.9709999412,1.415634726 \backslash \mathrm{C},-3.1295082535,-2.2703157289,0.6244381561 \backslash \mathrm{H}$, $-4.1026413176,-1.7892822521,0.5552469525 \backslash \mathrm{H},-2.8949463976,-2.9630764834$ $,-0.178119526 \backslash \mathrm{H},-2.8376312226,-2.5904190061,1.6197680232 \backslash \mathrm{C}, 0.509129341$ $4,-1.0139366798,-2.4069490822 \backslash \mathrm{H}, 1.1740902576,-1.7011016714,-2.93312282$ $94 \backslash \mathrm{H},-0.5207402858,-1.3801248812,-2.452086103 \backslash \mathrm{H}, 0.5695634161,-0.019106$ $2275,-2.8598286143 \backslash \mathrm{C}, 2.3793324744,-0.6631461637,1.4817697152 \backslash \mathrm{H}, 2.60087$ $49638,-0.964856986,2.5068973177 \backslash \mathrm{H}, 2.7511648117,-1.4127970315,0.7791557$ $268 \backslash \mathrm{H}, 2.84005151,0.3079815854,1.2734900071 \backslash \backslash$ Version=DEC-AXP-OSF / 1-G03R evB.03 $\backslash$ State $=2-\mathrm{A} \backslash \mathrm{HF}=-1447.794947 \backslash \mathrm{S} 2=0.76467 \backslash \mathrm{S} 2-1=0 . \backslash \mathrm{S} 2 \mathrm{~A}=0.750073 \backslash \mathrm{RMSD}=$ $4.821 \mathrm{e}-09 \backslash \mathrm{RMSF}=8.563 \mathrm{e}-06 \backslash \mathrm{Dipole}=0.6361618,-0.0299299,-0.2872864 \backslash \mathrm{Dipole}$ Deriv $=-0.0544053,0.6416475,-0.0869429,0.5145614,0.2108446,-0.1768354$,$0.1372428,-0.145465,-0.3965568,2.2557502,0.2252798,-0.2201342,-0.18452$ $12,1.8196482,0.1488033,0.364014,-0.2331095,1.7422238,-0.7519999,-0.678$ $012,0.1477965,-0.4987794,-1.0915076,0.1343792,0.1137868,0.2876396,-0.2$ $893508,0.065144,-0.1521721,-0.0752122,-0.0199353,0.2199822,0.0408434,-$ $0.0170461,0.167621,0.0889397,-1.5690664,0.1307421,-0.4994213,0.3508097$ $,-0.5672581,0.1855451,-0.6290539,0.2087988,-0.8983171,-0.7467175,0.121$ $8188,0.2061139,0.2780574,-0.695547,-0.4643815,0.1326,-0.3463904,-1.486$ $8222,0.0662062,0.0223003,0.0349713,0.0151951,-0.0937213,0.0221884,0.03$ $0459,0.000843,0.057577,-0.0350416,0.0654643,-0.0023535,-0.0017991,0.08$ $32767,-0.0341707,0.0006468,-0.0907238,0.0491729,0.0352732,0.0890048,0$. $0302059,0.0372921,0.0233368,-0.027218,0.0121596,-0.0827089,-0.0879126$, $-0.2131723,-0.306563,0.0618373,-0.3099903,-0.2852529,0.0837391,0.08749$ $83,0.0947631,0.0771853,-0.1042021,0.003393,0.0011703,-0.0401302,-0.030$ $994,0.0176834,0.0118407,0.0173209,0.0507675,-0.0038444,-0.0395616,0.02$ $69132,-0.0390618,-0.0702649,-0.0502863,0.0313851,-0.0433377,-0.0192597$ $,-0.0087521,-0.0490146,-0.0070316,-0.0510828,-0.0266318,0.0588589,-0.0$ $17464,0.0443913,-0.0462328,0.2152585,0.0536246,0.2280247,-0.042914,0.2$ $366916,0.018951,-0.1080843,0.1964426,0.9499412,0.0025814,0.0773582,0.0$ $606407,0.0777786,0.0025143,-0.0826699,0.040956,-0.0652443,-0.0472264,-$ $0.0485491,-0.0699693,-0.0799941,-0.0429678,0.0450583,0.0095535,0.03050$ $66,0.000956,0.0047644,0.0589411,-0.0205227,-0.0295155,-0.0162284,-0.05$ $63045,0.1041771,-0.0081156,0.0064558,-0.0206692,0.9528153,-0.1603526,0$ $.3141954,-0.0433729,0.2859733,0.0096484,0.0233393,-0.0040333,0.2925108$ $,-0.0344297,0.019138,-0.0559404,0.0424983,0.0649621,0.0520095,-0.08136$ $97,0.0497849,-0.0956734,-0.0521381,0.0330531,-0.0263686,0.0611635,-0.0$ $158271,-0.0540197,0.0694559,-0.0829974,0.0103354,-0.0296515,-0.0066566$ 
$,-0.0289551,-0.086573,-0.0589792,0.0032013,0.0497284,0.0189933,0.06460$ $31 \backslash$ Polar $=148.9191178,41.0738906,145.040224,-10.1587348,-6.7981237,92.0$ $910649 \backslash \mathrm{PG}=\mathrm{C} 01 \quad[\mathrm{X}(\mathrm{C} 4 \mathrm{H} 12 \mathrm{O} 2 \mathrm{P} 1 \mathrm{~S} 2)] \backslash \mathrm{NImag}=1 \backslash \backslash$

\section{$\mathrm{CH}_{3} \mathrm{SP} \cdot\left(\mathrm{OCH}_{3}\right)_{2} \mathrm{SCH}_{3}$}

$1 \backslash 1 \backslash G I N C-S C 33 \backslash F O p t \backslash U B 3 L Y P \backslash 6-31 G(d) \backslash C 4 H 12 O 2 P 1 S 2$ (2) \JOH501\11-Aug-2004 0 $\backslash \backslash \#$ B3LYP/6-31G* OPT FREQ MAXDISK=268435456\\ch3spsmeoch32_2 opt freq

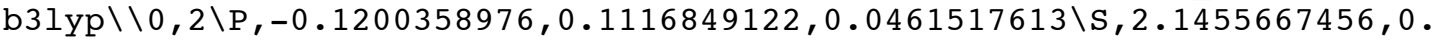
$2266232956,0.3184519261 \backslash 0,-0.6011564726,1.6290614675,-0.2856286195 \backslash 0,-$ $0.4480480343,0.0596264717,1.6525969355 \backslash \mathrm{C}, 2.6519875021,-0.0499249585,-1$ $.4248910892 \backslash \mathrm{H}, 2.3296863683,0.7709039012,-2.0701276165 \backslash \mathrm{H}, 2.2596551155,-$ $0.9959821114,-1.8066645796 \backslash \mathrm{H}, 3.7437780137,-0.0963702268,-1.4311728084 \backslash$ $\mathrm{S},-1.9527813501,-1.1474057835,-0.5803728052 \backslash \mathrm{C},-1.4531553534,-1.5666279$ $368,-2.2952606816 \backslash \mathrm{H},-2.1401734437,-2.3417823335,-2.6442072204 \backslash \mathrm{H},-0.435$ $297991,-1.9647394006,-2.3276908165 \backslash \mathrm{H},-1.5318111357,-0.6998971559,-2.95$ $57349705 \backslash \mathrm{C},-0.2072079826,-1.1310855144,2.4198711048 \backslash \mathrm{H},-0.5330321027,-0$ $.9056238088,3.4371332199 \backslash \mathrm{H},-0.7886438923,-1.9699676645,2.0243195301 \backslash \mathrm{H}$, $0.8580608166,-1.3830731756,2.4210999499 \backslash C,-0.310424434,2.7511307661,0$. $5692906772 \backslash \mathrm{H},-0.6932063541,3.6305614481,0.0481026995 \backslash \mathrm{H},-0.8156000733,2$ $.6348795918,1.5309159515 \backslash \mathrm{H}, 0.7689944775,2.8478794073,0.7226777125 \backslash \backslash$ Ver sion=DEC-AXP-OSF $/ 1-G 03 R e v B .03 \backslash$ State $=2-A \backslash H F=-1447.8200028 \backslash \mathrm{S} 2=0.757608 \backslash \mathrm{S}$ $2-1=0 . \backslash \mathrm{S} 2 \mathrm{~A}=0.750034 \backslash \mathrm{RMSD}=7.221 \mathrm{e}-09 \backslash \mathrm{RMSF}=1.859 \mathrm{e}-06 \backslash \mathrm{Dipole}=0.4674376,0.0$ $171462,-0.2333677 \backslash \mathrm{PG}=\mathrm{C} 01 \quad[\mathrm{X}(\mathrm{C} 4 \mathrm{H} 12 \mathrm{O} 2 \mathrm{P} 1 \mathrm{~S} 2)] \backslash \backslash @$

\section{$\mathrm{CH}_{3} \mathrm{SP}(\mathrm{H})\left(\mathrm{OCH}_{3}\right)_{2} \mathrm{SCH}_{3}$}

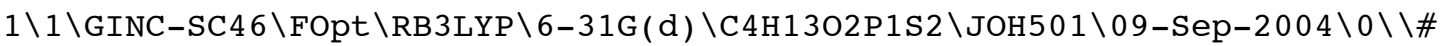
B3LYP/6-31G* OPT FREQ MAXDISK=268435456\\a_ch3spsmeoch32_2 opt freq b $31 \mathrm{yp} \backslash \backslash 0,1 \backslash \mathrm{P},-0.0471781684,0.3034909409,-0.0 \overline{6} 66236751 \backslash \mathrm{S}, 2 . \overline{0} 247521963,-0$ $.2741528826,-0.0287017394 \backslash 0,-0.402343806,1.8985205903,0.0554823705 \backslash 0,-$ $0.1228491314,0.0869736851,1.6131662876 \backslash \mathrm{C}, 2.4786323186,-0.2502913498,-1$ $.8025757956 \backslash \mathrm{H}, 3.5708720367,-0.2789654126,-1.8423676165 \backslash \mathrm{H}, 2.1347186651$, $0.6677343436,-2.2860577114 \backslash \mathrm{H}, 2.084025224,-1.1208424608,-2.3320166455 \backslash \mathrm{S}$ $,-1.6643775395,-1.0885923782,-0.3604893494 \backslash \mathrm{C},-1.8642748737,-1.05517770$ $97,-2.1803692529 \backslash \mathrm{H},-0.997655507,-1.485620633,-2.6875234051 \backslash \mathrm{H},-2.041013$ $0096,-0.0393379624,-2.5410481813 \backslash \mathrm{H},-2.7419934743,-1.6675915846,-2.4035$ $769467 \backslash \mathrm{C},-0.0493320938,-1.174678357,2.274871984 \backslash \mathrm{H}, 0.4176257179,-0.9970$ $620674,3.2486586159 \backslash \mathrm{H},-1.053091809,-1.5875181994,2.4271956222 \backslash \mathrm{H}, 0.5570$ $711137,-1.8994051083,1.7211204299 \backslash \mathrm{C},-0.6662317803,2.6157578878,1.26979$ $31087 \backslash \mathrm{H},-0.8584862971,3.6465953611,0.9611146088 \backslash \mathrm{H},-1.5415139177,2.2111$ $634842,1.7832915356 \backslash \mathrm{H}, 0.1940002992,2.5817964007,1.9419610214 \backslash \mathrm{H}, 0.02590$ $10489,0.5229968681,-1.4838483115 \backslash \backslash$ Version=DEC-AXP-OSF / 1-G03RevB.03\Sta $\mathrm{te}=1-\mathrm{A} \backslash \mathrm{HF}=-1448.4286677 \backslash \mathrm{RMSD}=5.400 \mathrm{e}-09 \backslash \mathrm{RMSF}=7.337 \mathrm{e}-05 \backslash \mathrm{Dipole}=-0.017372$ $3,-0.0728054,-0.5337924 \backslash \mathrm{PG}=\mathrm{C} 01 \quad[\mathrm{X}(\mathrm{C} 4 \mathrm{H} 1302 \mathrm{P} 1 \mathrm{~S} 2)] \backslash \backslash @$

\section{$\mathrm{S}=\mathrm{P}(\mathrm{CN})_{2} \mathrm{SCH}_{3}$}

$1 \backslash 1 \backslash G I N C-S C 81 \backslash F O p t \backslash R B 3 L Y P \backslash 6-31 G(d) \backslash C 3 H 3 N 2 P 1 S 2 \backslash J O H 501 \backslash 09-A u g-2004 \backslash 0 \backslash \backslash \#$ B3LYP /6-31G* OPT FREQ MAXDISK=268435456\\s=psmecn2_1 opt freq b3lyp $\backslash \backslash 0$ $, 1 \backslash \mathrm{S},-2.0670725635,-0.4234291283,-0.208855048 \backslash \mathrm{P},-0.1360167786,-0.30522$ $92824,-0.1315887138 \backslash \mathrm{S}, 0.8293250136,1.5750449811,-0.1797503837 \backslash \mathrm{C}, 0.5763$ $804466,-1.1633296217,1.276342306 \backslash \mathrm{C}, 0.7751174685,-1.1040740238,-1.45450$ $1096 \backslash \mathrm{C},-0.2021538827,2.4839934876,1.0425813308 \backslash \mathrm{H},-0.0123347667,2.13038$ $69495,2.0575589694 \backslash \mathrm{H},-1.2600880909,2.3939401413,0.7884060179 \backslash \mathrm{H}, 0.10833$ $04959,3.5275949687,0.9532481824 \backslash \mathrm{N}, 1.3130490027,-1.6265046721,-2.342903$ $2606 \backslash N, 0.9886996609,-1.6876204022,2.2294417175 \backslash \backslash$ Version=DEC-AXP-OSF / 1G03RevB.03 \State $=1-A \backslash H F=-1363.3217499 \backslash$ RMSD $=9.848 e-09 \backslash R M S F=1.436 e-05 \backslash D i$ pole $=-0.2052502,1.6324148,0.4573093 \backslash P G=C 01[X(C 3 H 3 N 2 P 1 S 2)] \backslash \backslash @$

\section{TS: $\cdot \mathrm{CH}_{3}+\mathrm{S}=\mathrm{P}(\mathrm{CN})_{2} \mathrm{SCH}$}

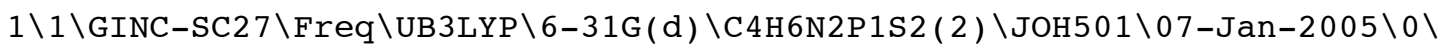
$\backslash \#$ B3LYP /6-31G* OPT=(TS, CALCALL, NOEIGENTEST, MAXCYC=200) MAXDISK=134217 728\\ts_ch3spsmecn2_up1_6db3 transition state search $\backslash \backslash 0,2 \backslash S,-1.7694936$ $626,-0 . \overline{1} 737746832,-\overline{0} .00 \overline{2} 7742615 \backslash \mathrm{P}, 0.2211775234,-0.0793521766,-0.042207$ 
$2526 \backslash S, 1.4076307949,1.6332381399,-0.590538961 \backslash C, 0.5645890335,2.8891573$ $991,0.455700315 \backslash \mathrm{C}, 0.9537733738,-0.6771836408,1.484904815 \backslash \mathrm{C}, 0.949828686$ $2,-1.2423686873,-1.1957628123 \backslash \mathrm{H}, 0.9419639228,3.8547398988,0.1112167051$ $\backslash \mathrm{H},-0.5170291236,2.8504545999,0.307146883 \backslash \mathrm{H}, 0.8146732988,2.7553753863$, $1.5098941513 \backslash \mathrm{C},-3.5933119936,-1.7982295416,-0.0139712951 \backslash \mathrm{H},-4.38083840$ $74,-1.0843321186,-0.234081778 \backslash \mathrm{H},-3.2746869799,-2.4532993398,-0.8164800$ $436 \backslash \mathrm{H},-3.5246069121,-2.1736328572,1.0005670966 \backslash \mathrm{N}, 1.3706121266,-0.99170$ $51656,2.5251016768 \backslash \mathrm{N}, 1.3670153927,-1.9995364635,-1.9732972199 \backslash \backslash$ Version $=\mathrm{DEC}-\mathrm{AXP}-\mathrm{OSF} / 1-\mathrm{G} 03 \mathrm{RevB} .03 \backslash \mathrm{State}=2-\mathrm{A} \backslash \mathrm{HF}=-1403.1556975 \backslash \mathrm{S} 2=0.767278 \backslash \mathrm{S} 2-1=$ $0 . \backslash \mathrm{S} 2 \mathrm{~A}=0.750096 \backslash \mathrm{RMSD}=8.120 \mathrm{e}-09 \backslash \mathrm{RMSF}=1.704 \mathrm{e}-06 \backslash \mathrm{Dipole}=-0.836168,1.20541$ $67,0.0906963 \backslash$ DipoleDeriv $=0.3016962,0.5946882,0.0215053,0.5387126,0.003$ $6247,-0.006235,-0.004125,-0.005399,-0.3935683,1.670127,0.166615,-0.038$ $883,0.0516122,1.4806554,-0.055207,-0.2936739,-0.2274485,1.2749729,-0.8$ $974589,-0.6328257,0.0678198,-0.5323314,-0.7224718,0.0529263,0.2378788$, $0.2197065,-0.1829901,0.0410105,-0.1127341,-0.0538363,0.0244187,0.17688$ $73,0.0949361,0.0399511,0.2198134,0.1151589,-0.2631805,0.0242373,-0.226$ $9165,0.1923772,-0.1527179,0.2053118,0.0360565,0.1335808,-0.4175959,-0$. $2293599,0.1947486,0.1942969,0.093915,-0.3878495,-0.2625176,0.0849675,-$ $0.1800485,-0.3077203,0.0889783,-0.0075607,0.0360946,-0.0167427,-0.0364$ $338,0.0005892,0.0276591,-0.0349976,0.063462,-0.0106932,0.0679984,0.006$ $5451,0.0046843,0.0754596,-0.0334256,-0.0215406,-0.1059155,0.0410164,0$. $0722362,0.0794222,0.0128455,0.0332175,0.0194269,-0.0154339,-0.0135458$, $-0.0662814,-0.0688945,-0.4144587,-0.3125746,-0.0135603,-0.358198,-0.17$ $57159,-0.0117072,0.0058723,-0.0014532,0.0892616,-0.0269614,0.0326885,-$ $0.0148128,-0.0142988,-0.0034413,0.0106788,-0.0063764,0.0155831,0.05326$ $98,0.007632,-0.0146789,0.0153541,0.0006345,0.0075508,-0.0415828,0.0342$ $852,-0.0288725,0.0105664,0.0061371,-0.0302425,0.0018007,-0.0254768,0.0$ $251141,0.0339587,-0.0274574,0.0157797,-0.0174365,-0.1840384,0.0049149$, $0.0523344,-0.0126694,-0.1974213,-0.051399,-0.1210545,-0.0280954,-0.132$ $5092,-0.1616662,-0.0546968,-0.0605877,0.020145,-0.1126672,0.0791074,0$. $0211031,0.0740482,-0.1269933 \backslash$ Polar $=169.5563617,39.5714886,130.5051028$, $-2.3703376,3.3375595,95.8641289 \backslash \mathrm{PG}=\mathrm{C} 01 \quad[\mathrm{X}(\mathrm{C} 4 \mathrm{H} 6 \mathrm{~N} 2 \mathrm{P} 1 \mathrm{~S} 2)] \backslash \mathrm{NImag}=1 \backslash \backslash$

\section{$\mathrm{CH}_{3} \mathrm{SP} \cdot(\mathrm{CN})_{2} \mathrm{SCH}_{3}$}

$1 \backslash 1 \backslash G I N C-S C 6 \backslash F O p t \backslash U B 3 L Y P \backslash 6-31 G(d) \backslash C 4 H 6 N 2 P 1 S 2$ ( 2$) \backslash J O H 501 \backslash 09-A u g-2004 \backslash 0 \backslash \backslash$ \# B3LYP/6-31G* OPT FREQ MAXDISK=268435456\\ch3spsmecn2_1 opt freq b3ly $\mathrm{p} \backslash \backslash 0,2 \backslash \mathrm{P}, 0.0021097357,-0.0054708181,-0.0071305652 \backslash \mathrm{S}, 2 . \overline{2} 914944048,-0.03$ $78662848,0.131851724 \backslash \mathrm{C},-0.2409688921,1.7689114113,-0.0027312777 \backslash \mathrm{C},-0.2$ $069471818,-0.414447302,1.7239133464 \backslash \mathrm{C}, 2.6573015152,0.6118727514,-1.543$ $9810756 \backslash \mathrm{H}, 2.2827090786,1.631407132,-1.6642147635 \backslash \mathrm{H}, 2.2474424684,-0.039$ $6859849,-2.3199372494 \backslash \mathrm{H}, 3.7465273586,0.6197385738,-1.634449349 \backslash \mathrm{S},-2.13$ $88744419,-0.4232736903,-0.7182343041 \backslash \mathrm{C},-2.1162563448,-2.2481525131,-0$. $5349490006 \backslash \mathrm{H},-3.0920836062,-2.5992094782,-0.8801754156 \backslash \mathrm{H},-1.9820236015$ $,-2.5399042125,0.5096019039 \backslash \mathrm{H},-1.3418523202,-2.6997803137,-1.160226707$ $9 \backslash \mathrm{N},-0.3454743787,2.921324786,-0.1247073207 \backslash \mathrm{N},-0.3535341066,-0.8100919$ $188,2.8082743758 \backslash \backslash$ Version=DEC-AXP-OSF $/ 1-G 03$ RevB.03 $\backslash$ State $=2-A \backslash H F=-1403$. $1944816 \backslash \mathrm{S} 2=0.760056 \backslash \mathrm{S} 2-1=0 . \backslash \mathrm{S} 2 \mathrm{~A}=0.750045 \backslash \mathrm{RMSD}=9.484 \mathrm{e}-09 \backslash \mathrm{RMSF}=6.080 \mathrm{e}-06$ $\backslash$ Dipole $=0.3629985,-1.0946707,-1.3912667 \backslash P G=C 01 \quad[X(C 4 H 6 N 2 P 1 S 2)] \backslash \backslash @$

\section{$\mathrm{CH}_{3} \mathrm{SP}(\mathrm{H})(\mathrm{CN})_{2} \mathrm{SCH}_{3}$}

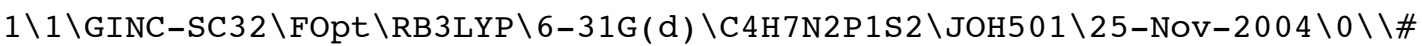
B3LYP/6-31G* OPT FREQ MAXDISK=268435456\\a3_ch3spsmecn2_1 opt freq b31 $\mathrm{yp} \backslash \backslash 0,1 \backslash \mathrm{P}, 0.0004354917,0.1774658499,0.04237 \overline{7} 1216 \backslash \mathrm{S}, 1.91 \overline{6} 5765473,-0.717$ $2651921,-0.1578589446 \backslash \mathrm{C}, 0.0047136752,1.9107799918,0.5667787865 \backslash \mathrm{C}, 0.015$ $3359236,-0.409105063,1.8554172172 \backslash \mathrm{C}, 2.3530996903,-0.3075878693,-1.8915$ $558717 \backslash \mathrm{H}, 2.4301413133,0.7712023202,-2.0391883628 \backslash \mathrm{H}, 1.6424441519,-0.742$ $0721001,-2.5975459585 \backslash \mathrm{H}, 3.3350276495,-0.7591882675,-2.0569835249 \backslash \mathrm{S},-1$. $918985246,-0.7168230915,-0.1261720773 \backslash \mathrm{C},-2.3839744289,-0.3069268416,-1$ $.8523904895 \backslash \mathrm{H},-3.3680803579,-0.7593032693,-2.0019856423 \backslash \mathrm{H},-1.684536824$ $6,-0.7406042263,-2.5699912328 \backslash \mathrm{H},-2.464237647,0.7718493731,-1.998398270$ $2 \backslash \mathrm{N}, 0.0072013193,3.0458627244,0.8210475261 \backslash \mathrm{N}, 0.0238129557,-0.784969458$ $4,2.9574523487 \backslash \mathrm{H},-0.0109005673,0.5923267904,-1.3460644606 \backslash \backslash$ Version=DEC $-\mathrm{AXP}-\mathrm{OSF} / 1-\mathrm{G} 03 \mathrm{RevB}$. 03 \State $=1-\mathrm{A} \backslash \mathrm{HF}=-1403.7922585 \backslash \mathrm{RMSD}=6.311 \mathrm{e}-09 \backslash \mathrm{RMSF}=1$ 
$.113 e-05 \backslash \mathrm{Dipole}=-0.0234005,-0.4885585,-2.8623565 \backslash \mathrm{PG}=\mathrm{C} 01 \quad[\mathrm{X}(\mathrm{C} 4 \mathrm{H} 7 \mathrm{~N} 2 \mathrm{P} 1 \mathrm{~S} 2)$ ]$\backslash \backslash$ Q

\section{$\mathrm{S}=\mathrm{P}\left(\mathrm{OCH}_{3}\right)(\mathrm{CN}) \mathrm{SCH}_{3}$}

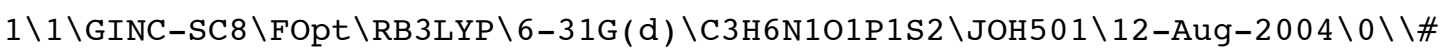
B3LYP/6-31G* OPT FREQ MAXDISK=268435456\\s=psmeoch $3 \mathrm{cn} 3$ opt freq b3ly $\mathrm{p} \backslash \backslash 0,1 \backslash \mathrm{S},-1.98446645,-0.2372834139,0.2797322454 \backslash \mathrm{P},-0.0 \overline{6} 92884381,-0.343$ $3613936,0.0028880095 \backslash \mathrm{S}, 1.0211726086,1.4688290233,-0.0763076042 \backslash \mathrm{C}, 0.766$ $8636475,-1.3392853934,1.2360641509 \backslash 0,0.5154889553,-1.1262115584,-1.290$ $0615101 \backslash \mathrm{C}, 0.0060359642,2.5763077192,0.982083832 \backslash \mathrm{H}, 0.1220310222,2.32172$ $15368,2.0367658102 \backslash \mathrm{H},-1.0436368135,2.524609888,0.6886731366 \backslash \mathrm{H}, 0.392711$ $3631,3.5823014925,0.801916258 \backslash \mathrm{N}, 1.2679428382,-1.9554192155,2.085268799$ $8 \backslash \mathrm{C},-0.0326927719,-0.8722597953,-2.6037620305 \backslash \mathrm{H}, 0.4917932209,-1.555260$ $2099,-3.2720747757 \backslash \mathrm{H},-1.1053420099,-1.0817515408,-2.6127932638 \backslash \mathrm{H}, 0.153$ $7187035,0.1631217801,-2.9033068008 \backslash \backslash$ Version=DEC-AXP-OSF / 1-G03RevB.03 \S tate $=1-\mathrm{A} \backslash \mathrm{HF}=-1385.6491941 \backslash \mathrm{RMSD}=4.622 \mathrm{e}-09 \backslash \mathrm{RMSF}=8.541 \mathrm{e}-06 \backslash \mathrm{Dipole}=-0.0747$ $084,1.2157907,-1.1963432 \backslash \mathrm{PG}=\mathrm{C} 01 \quad[\mathrm{X}(\mathrm{C} 3 \mathrm{H} 6 \mathrm{~N} 101 \mathrm{P} 1 \mathrm{~S} 2)] \backslash \backslash @$

\section{TS: $\cdot \mathrm{CH}_{3}+\mathrm{S}=\mathrm{P}\left(\mathrm{OCH}_{3}\right)(\mathrm{CN}) \mathrm{SCH}_{3}$}

$1 \backslash 1 \backslash G I N C-S C 160 \backslash F r e q \backslash U B 3 L Y P \backslash 6-31 G(d) \backslash C 4 H 9 N 101 P 1 S 2$ ( 2) \JOH501\26-Nov-2004 $\backslash 0 \backslash \backslash \#$ B3LYP/6-31G* FREQ MAXDISK=268435456\\ts_ch3spsmeoch3cn_10 g98 tr ansition state search $\backslash \backslash 0,2 \backslash S,-1.3730369789,-0.8951108526,-0 . \overline{5} 473530526$ $\backslash \mathrm{P}, 0.1602030211,0.2933881474,-0.0555970526 \backslash \mathrm{S}, 2.0813290211,-0.462629852$ $6,0.5398689474 \backslash \mathrm{C}, 2.1843880211,-1.9593038526,-0.5231160526 \backslash \mathrm{C}, 0.47344902$ $11,1.5606481474,-1.2839400526 \backslash 0,-0.1295369789,1.3122131474,1.180769947$ $4 \backslash \mathrm{H}, 3.0157540211,-2.5479278526,-0.1278370526 \backslash \mathrm{H}, 1.2619180211,-2.5400808$ $526,-0.4583820526 \backslash \mathrm{H}, 2.3910880211,-1.6885118526,-1.5600740526 \backslash \mathrm{C},-3.6999$ $549789,-0.7807658526,-0.3564790526 \backslash \mathrm{H},-3.9537639789,-1.4093598526,-1.20$ $54730526 \backslash \mathrm{H},-3.8773129789,-1.2073378526,0.6258569474 \backslash \mathrm{H},-3.9087569789,0$. $2777331474,-0.4699290526 \backslash \mathrm{C},-0.6423299789,0.8082851474,2.4316569474 \backslash \mathrm{H},-$ $0.7490319789,1.6823281474,3.0749099474 \backslash \mathrm{H},-1.6147139789,0.3313591474,2$. $2806009474 \backslash \mathrm{H}, 0.0581100211,0.0978221474,2.8808319474 \backslash \mathrm{N}, 0.6834250211,2.2$ $937311474,-2.1630990526 \backslash \backslash$ Version=DEC-AXP-OSF $/ 1-G 98 R e v A .11 .3 \backslash \mathrm{HF}=-1425.4$ $779379 \backslash \mathrm{S} 2=0.766364 \backslash \mathrm{S} 2-1=0 . \backslash \mathrm{S} 2 \mathrm{~A}=0.750084 \backslash \mathrm{RMSD}=5.757 \mathrm{e}-09 \backslash \mathrm{RMSF}=8.584 \mathrm{e}-06 \backslash$ Dipole $=-0.3386148,-1.0695868,1.2541458 \backslash$ DipoleDeriv=0.69238, -0.0190309 , $-0.0847017,0.0486717,-0.4447884,-0.0358388,-0.0601859,0.0291164,-0.433$ $942,1.6519347,-0.0995489,0.1276648,0.0073148,1.5978469,0.2627611,0.561$ $9086,0.0518886,1.6219513,-1.232123,0.2273881,-0.1284993,0.1002585,-0.2$ $627763,0.0090177,-0.5232311,0.0774799,-0.2722088,0.0625169,-0.029871,-$ $0.0141174,-0.1446004,0.1646843,0.0915549,-0.1243555,0.138584,0.1130848$ $,-0.0693029,-0.0006801,0.0425139,-0.1806709,-0.3681128,0.3134557,-0.17$ $76781,0.0559318,-0.2806521,-0.5418464,0.0828799,0.3043121,0.0678864,-0$ $.7516264,-0.3087446,0.1618716,-0.2910627,-1.4856463,0.0124352,0.076246$ $3,-0.0306976,0.0832701,0.0042962,-0.0144251,-0.0144409,-0.0279846,0.05$ $1007,0.042984,-0.0824919,0.0129101,-0.0260668,0.0080773,-0.0347123,0.0$ $680361,-0.0766338,0.0381022,0.1020585,-0.0080533,0.0154344,0.0307987,-$ $0.0162737,-0.0226889,0.0748463,-0.0483115,-0.0760913,-0.6164666,-0.078$ $7768,-0.0034781,-0.0265391,0.0376189,-0.0297107,0.0212201,-0.034936,0$. $0865316,-0.0532492,-0.0482525,-0.0341378,-0.0031871,0.0265048,-0.04442$ $08,0.0044346,-0.0410139,-0.0009694,-0.055989,-0.020614,0.0242527,0.004$ $2024,0.0399161,0.0366692,0.0154141,0.036473,-0.0426896,-0.0431419,0.02$ $33981,0.0013421,0.0052707,-0.0423844,0.0091045,0.0122448,0.0064202,0.0$ $532744,0.2633343,-0.0503749,-0.2487132,0.0610531,0.2172298,-0.2497503$, $-0.1444614,0.1144102,0.8729913,0.084491,0.0101185,0.0318481,0.0121679$, $-0.0255941,-0.0797874,0.0288574,-0.0670155,-0.0464775,-0.0518086,-0.03$ $48457,0.0288851,-0.0682761,0.0467772,0.059832,-0.0485725,-0.0325238,0$. $0025665,-0.0110628,0.0693917,-0.0381376,0.0445283,0.0089015,0.1001997$, $0.0050339,0.0119829,-0.0105933,-0.2371439,-0.0168826,-0.0066806,-0.016$ $0822,-0.2402968,-0.062516,0.1390576,0.0971945,-0.1902387 \backslash$ Polar $=184.185$ $5936,9.1324126,104.9345951,4.7518235,-3.0899647,97.7694483 \backslash \mathrm{PG}=\mathrm{C} 01] \mathrm{X}(\mathrm{C}$ 4 H9N1O1P1S2 ) ] \NImag=1\\ 


\section{$\mathrm{CH}_{3} \mathrm{SP} \cdot\left(\mathrm{OCH}_{3}\right)(\mathrm{CN}) \mathrm{SCH}_{3}$}

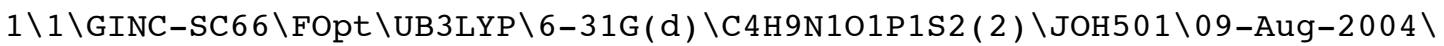
$0 \backslash \backslash \#$ B3LYP/6-31G* OPT FREQ MAXDISK=268435456\\ch3spsmeoch3cn_1 opt fre q b3lyp $\backslash \backslash 0,2 \backslash P, 0.0388318776,-0.0459676043,0.0298170953 \backslash S, 2.3 \overline{6} 4072268$, $0.1770821307,-0.1123377895 \backslash C,-0.1647223804,1.7480758543,-0.0776039484 \backslash$ $0,0.0859672199,-0.3565528117,1.6336517796 \backslash C, 2.5197000599,0.1178099545$, $-1.9155699968 \backslash \mathrm{H}, 2.1706131781,1.116295597,-2.1913484393 \backslash \mathrm{H}, 1.9832677142$, $-0.6397712358,-2.4928006999 \backslash \mathrm{H}, 3.5848930768,0.040573725,-2.1493557302 \backslash \mathrm{S}$ $,-2.0937612305,-0.4318048412,-0.5404171525 \backslash \mathrm{C},-1.9742389943,-2.25650399$ $05,-0.7331411085 \backslash \mathrm{H},-2.9506575053,-2.5931529601,-1.0894430776 \backslash \mathrm{H},-1.7534$ $205421,-2.7436434481,0.2190737329 \backslash \mathrm{H},-1.2166037798,-2.5232726757,-1.474$ $1258871 \backslash \mathrm{C},-0.8096313865,0.2488202306,2.5822416057 \backslash \mathrm{H},-0.5280735475,-0.1$ $574047502,3.5549606277 \backslash \mathrm{H},-0.6843200332,1.3367767365,2.5815903851 \backslash \mathrm{H},-1$. $8488697405,-0.0051492985,2.3539174269 \backslash \mathrm{N},-0.2540950194,2.9005234463,-0$. $2172027492 \backslash \backslash$ Version=DEC-AXP-OSF / 1-G03RevB.03\State $=2-\mathrm{A} \backslash \mathrm{HF}=-1425.507240$ $6 \backslash S 2=0.758035 \backslash S 2-1=0 . \backslash S 2 A=0.750033 \backslash R M S D=7.215 e-09 \backslash R M S F=3.870 e-06 \backslash D i p o l$ $\mathrm{e}=-0.3661908,-1.2598822,0.0530398 \backslash \mathrm{PG}=\mathrm{C} 01 \quad[\mathrm{X}(\mathrm{C} 4 \mathrm{H} 9 \mathrm{~N} 101 \mathrm{P} 1 \mathrm{~S} 2)] \backslash \backslash @$

\section{$\mathrm{CH}_{3} \mathrm{SP}(\mathrm{H})\left(\mathrm{OCH}_{3}\right)(\mathrm{CN}) \mathrm{SCH}_{3}$}

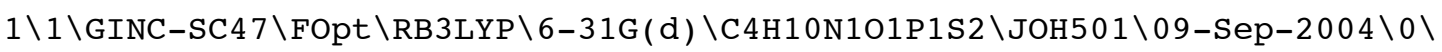
$\backslash \#$ B3LYP/6-31G* OPT FREQ MAXDISK=268435456\\a ch3spsmeoch3cn 1 opt fre q b3lyp $\backslash \backslash 0,1 \backslash P, 0.0299324068,0.1337514628,-0.0 \overline{2} 15521219 \backslash S, 2.1 \overline{5} 30190264$, $0.131160327,0.043935194 \backslash \mathrm{C},-0.7808487772,1.7637484242,-0.1572830344 \backslash 0,0$ $.1135845917,0.1583489347,1.6675627094 \backslash \mathrm{C}, 2.5775854599,-0.0452112977,-1$. $7299274805 \backslash \mathrm{H}, 2.1863660154,0.7835996226,-2.3240882412 \backslash \mathrm{H}, 2.2315241451,-0$ $.9984602364,-2.1360139376 \backslash \mathrm{H}, 3.6701039278,-0.0227349424,-1.7742839769 \backslash \mathrm{S}$ $,-1.2779548223,-1.5528096712,0.0413359862 \backslash \mathrm{C},-1.7351568364,-1.776820176$ $2,-1.718880384 \backslash \mathrm{H},-2.3591780974,-2.6739346955,-1.7556323891 \backslash \mathrm{H},-0.852990$ $0443,-1.9366728129,-2.3431045153 \backslash \mathrm{H},-2.3118357215,-0.9284214427,-2.0931$ $054008 \backslash \mathrm{C},-0.9392196057,0.6247386705,2.5152063119 \backslash \mathrm{H},-0.5896756737,0.474$ $6083847,3.5393940829 \backslash \mathrm{H},-1.1372142817,1.6906909567,2.3547001457 \backslash \mathrm{H},-1.86$ $32766151,0.0555133986,2.3636344064 \backslash \mathrm{N},-1.3008856464,2.7927600638,-0.314$ $5832926 \backslash \mathrm{H}, 0.0395243247,0.0810836826,-1.4656683337 \backslash \backslash$ Version=DEC-AXP-OSF /1-G03RevB.03 \State $=1-\mathrm{A} \backslash \mathrm{HF}=-1426.1129042 \backslash \mathrm{RMSD}=5.624 \mathrm{e}-09 \backslash \mathrm{RMSF}=3.348 \mathrm{e}-05$ $\backslash$ Dipole $=0.0912686,-1.0003106,-0.8942721 \backslash \mathrm{PG}=\mathrm{C} 01$ [X(C4H10N1O1P1S2)] \\

\section{$\mathrm{S}=\mathrm{P}\left(\mathrm{CH}_{3}\right)_{3}$}

$1 \backslash 1 \backslash G I N C-S C 32 \backslash F O p t \backslash R B 3 L Y P \backslash 6-31 G(d) \backslash C 3 H 9 P 1 S 1 \backslash J O H 501 \backslash 28-J u 1-2004 \backslash 1 \backslash \backslash \#$ B3 LYP/6-31G* FOPT=Z-MATRIX FREQ MAXDISK=65536000\\s=pme3-test $\backslash \backslash 0,1 \backslash P \backslash S, 1$ , $\mathrm{B} 1 \backslash \mathrm{C}, 1, \mathrm{~B} 2,2, \mathrm{~A} 1 \backslash \mathrm{C}, 1, \mathrm{~B} 2,2, \mathrm{~A} 1,3,-120$, $0 \backslash \mathrm{C}, 1, \mathrm{~B} 2,2, \mathrm{~A} 1,3,120$, $0 \backslash \mathrm{H}, 3, \mathrm{~B} 3,1, \mathrm{~A} 2$ $, 2,-180 ., 0 \backslash \mathrm{H}, 4, \mathrm{~B} 3,1, \mathrm{~A} 2,2,180.0 \backslash \mathrm{H}, 5, \mathrm{~B} 3,1, \mathrm{~A} 2,2,-180 ., 0 \backslash \mathrm{H}, 3, \mathrm{~B} 4,1, \mathrm{~A} 3,6, \mathrm{D} 1$ $, 0 \backslash \mathrm{H}, 3, \mathrm{~B} 4,1, \mathrm{~A} 3,6,-\mathrm{D} 1,0 \backslash \mathrm{H}, 4, \mathrm{~B} 4,1, \mathrm{~A} 3,7,-\mathrm{D} 1,0 \backslash \mathrm{H}, 4, \mathrm{~B} 4,1, \mathrm{~A} 3,7, \mathrm{D} 1,0 \backslash \mathrm{H}, 5, \mathrm{~B} 4,1$ $, \mathrm{A} 3,8, \mathrm{D} 1,0 \backslash \mathrm{H}, 5, \mathrm{~B} 4,1, \mathrm{~A} 3,8,-\mathrm{D} 1,0 \backslash \backslash \mathrm{B} 1=1.97181801 \backslash \mathrm{B} 2=1.84065056 \backslash \mathrm{B} 3=1.09581$ $456 \backslash \mathrm{B} 4=1.09355316 \backslash \mathrm{A} 1=114.60482104 \backslash \mathrm{A} 2=111.32520613 \backslash \mathrm{A} 3=109.05555001 \backslash \mathrm{D} 1=1$ $20.91062736 \backslash \backslash$ Version=DEC-AXP-OSF $/ 1-G 03$ RevB . 03 $\backslash$ State $=1-A 1 \backslash H F=-859.32233$ $2 \backslash \mathrm{RMSD}=5.439 \mathrm{e}-09 \backslash \mathrm{RMSF}=1.767 \mathrm{e}-05 \backslash \mathrm{Dipole}=0 ., 0,-2.0493024 \backslash \mathrm{PG}=\mathrm{C} 03 \mathrm{~V} \quad[\mathrm{C} 3(\mathrm{P} 1$ $\mathrm{S} 1), 3 \mathrm{SGV}(\mathrm{C} 1 \mathrm{H} 1), \mathrm{X}(\mathrm{H} 6)] \backslash \backslash @$

\section{TS: $\cdot \mathrm{CH}_{3}+\mathrm{S}=\mathrm{P}\left(\mathrm{CH}_{3}\right)_{3}$}

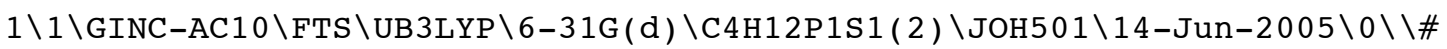
UB3LYP /6-31G* OPT $=(T S, C A L C F C$, NOEIGENTEST, MAXCYC $=200)$ FREQ=NORAMAN MAX

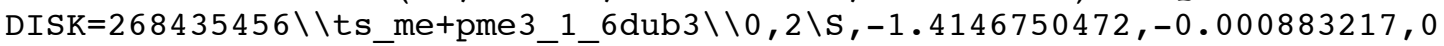
$.2537474491 \backslash \mathrm{P}, 0.70 \overline{3} 1707058,0.213247784,0.1193005087 \backslash \mathrm{C}, 1.4355600258,1.9$ $066282384,-0.0559028344 \backslash \mathrm{C}, 1.7027952106,-0.5753518887,1.4590475924 \backslash \mathrm{C}, 1$. $2806417701,-0.6448811194,-1.4040075668 \backslash \mathrm{H}, 0.9792789339,-1.6953336266,-1$ $.3612802183 \backslash \mathrm{H}, 2.3693432068,-0.5829135754,-1.5125458123 \backslash \mathrm{H}, 0.8030124756$, $-0.187126919,-2.2751040452 \backslash \mathrm{H}, 1.3667297081,-1.6084766073,1.5853163695 \backslash \mathrm{H}$ $, 1.5297426848,-0.0416207999,2.3980216123 \backslash \mathrm{H}, 2.7752473369,-0.567973139,1$ $.2299096701 \backslash \mathrm{C},-3.1387194729,-1.0027923362,-0.5934526404 \backslash \mathrm{H},-3.956504073$ $8,-0.4921304557,-0.0850581181 \backslash \mathrm{H},-3.1166544054,-0.8213899147,-1.6659340$ $365 \backslash \mathrm{H},-3.0489800075,-2.0533457149,-0.324917674 \backslash \mathrm{H}, 2.512316443,1.8690896$ $53,-0.2629493063 \backslash \mathrm{H}, 0.9285071372,2.4251061351,-0.8748277773 \backslash \mathrm{H}, 1.2635355$ 
$271,2.4699123124,0.8657952163 \backslash \backslash$ Version=IA64L-G03RevC . 02 $\backslash$ State $=2-A \backslash H F=-$ $899.1417963 \backslash \mathrm{S} 2=0.760471 \backslash \mathrm{S} 2-1=0 . \backslash \mathrm{S} 2 \mathrm{~A}=0.750043 \backslash \mathrm{RMSD}=3.347 \mathrm{e}-09 \backslash \mathrm{RMSF}=3.554$ e-06\Dipole $=1.7354125,-0.0062704,-0.3136778 \backslash \mathrm{PG}=\mathrm{C} 01 \quad[\mathrm{X}(\mathrm{C} 4 \mathrm{H} 12 \mathrm{P} 1 \mathrm{~S} 1)] \backslash \backslash @$

\section{-P( $\left(\mathrm{CH}_{3}\right)_{3} \mathrm{SCH}_{3}$}

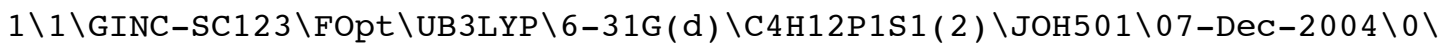
\\# B3LYP/6-31G* OPT FREQ MAXDISK=134217728\\pme3sch3 ax2 opt freq b3ly $\mathrm{p} \backslash \backslash 0,2 \backslash \mathrm{S},-1.6892453881,0.8959415254,-0.4716064264 \backslash \mathrm{P}, \overline{0} .688127042,-0.190$ $0068524,0.2853298364 \backslash \mathrm{C}, 1.5498682376,1.4468920022,0.2078980152 \backslash \mathrm{C}, 1.7413$ $197763,-1.0782182442,1.5551412346 \backslash \mathrm{C}, 1.252189797,-1.0035109571,-1.28223$ $33444 \backslash \mathrm{H}, 0.9250422197,-2.0483298262,-1.2945679871 \backslash \mathrm{H}, 2.3434937537,-0.974$ $1317631,-1.3863770509 \backslash \mathrm{H}, 0.7906539201,-0.4916673873,-2.1308368508 \backslash \mathrm{H}, 1.4$ $285029131,-2.1251396384,1.6221123252 \backslash \mathrm{H}, 1.5938503698,-0.6172839001,2.53$ $70190047 \backslash \mathrm{H}, 2.8090958235,-1.042123899,1.3032919286 \backslash \mathrm{H}, 2.627536912,1.3262$ $256656,0.0491020971 \backslash \mathrm{H}, 1.1302935248,2.0422307444,-0.6077846704 \backslash \mathrm{H}, 1.3852$ $326587,1.9912313068,1.1429658109 \backslash \mathrm{C},-2.7626300162,-0.5431003294,-0.1179$ $117639 \backslash \mathrm{H},-3.7881716892,-0.2569820332,-0.3738368837 \backslash \mathrm{H},-2.488488224,-1.4$ $169435277,-0.7161944283 \backslash \mathrm{H},-2.7355083715,-0.8044221908,0.9434971339 \backslash \backslash \mathrm{Ve}$ rsion=DEC-AXP-OSF $/ 1-G 03 R e v B .03 \backslash$ State $=2-A \backslash H F=-899.1679327 \backslash \mathrm{S} 2=0.755196 \backslash \mathrm{S}$ $2-1=0 . \backslash S 2 A=0.750014 \backslash R M S D=3.485 e-09 \backslash R M S F=3.046 e-06 \backslash D i p o l e=1.1242204,-0$. $7882041,0.2777739 \backslash \mathrm{PG}=\mathrm{C} 01 \quad[\mathrm{X}(\mathrm{C} 4 \mathrm{H} 12 \mathrm{P} 1 \mathrm{~S} 1)] \backslash \backslash @$

\section{$\mathrm{HP}\left(\mathrm{CH}_{3}\right)_{3} \mathrm{SCH}_{3}$}

$1 \backslash 1 \backslash G I N C-S C 31 \backslash F O p t \backslash R B 3 L Y P \backslash 6-31 G(d) \backslash C 4 H 13 P 1 S 1 \backslash J O H 501 \backslash 14-D e c-2004 \backslash 0 \backslash \backslash \# B$ 3LYP/6-31G* OPT FREQ MAXDISK $=65536000 \backslash \backslash \mathrm{a} \_$pme3sch3_ax2 opt freq b3lyp $\backslash \backslash$ $0,1 \backslash S,-1.75173029,0.179242632,-0.2322394 \overline{394} \backslash \mathrm{P}, 0.6 \overline{8} 97553746,0.077690516$ $4,0.1966866427 \backslash \mathrm{C}, 0.6098913466,1.9186713726,0.3276708279 \backslash \mathrm{C}, 2.4689461386$ $,-0.1022245001,0.8326498138 \backslash \mathrm{C}, 0.9686086919,-0.5973656383,-1.4961001969$ $\backslash \mathrm{H}, 0.5448423842,-1.6043269046,-1.5384312306 \backslash \mathrm{H}, 2.0351315794,-0.63857020$ $51,-1.7404901888 \backslash \mathrm{H}, 0.4217350582,0.0180441012,-2.2118553352 \backslash \mathrm{H}, 2.7525240$ $645,-1.16190909,0.837449038 \backslash \mathrm{H}, 2.5381905594,0.2654236452,1.8640609719 \backslash \mathrm{H}$ , 3.1949936716,0.4474028417,0.2223955513\H,1.6100849505,2.3570527666,0. $2598311101 \backslash \mathrm{H},-0.0411819135,2.3134391987,-0.454900776 \backslash \mathrm{H}, 0.1635366202,2$. $1695014161,1.2934837982 \backslash \mathrm{C},-2.195803669,-1.5120723941,0.2976480646 \backslash \mathrm{H},-3$ $.1498308465,-1.4919348435,0.8319987323 \backslash \mathrm{H},-2.2853952577,-2.1998370733,-$ $0.5498503922 \backslash \mathrm{H},-1.4414060137,-1.9202486785,0.9919974323 \backslash \mathrm{H}, 0.2282741165$ $,-0.8293300748,1.1886316218 \backslash \backslash$ Version=DEC-AXP-OSF / 1-G03RevB.03 \State=1$\mathrm{A} \backslash \mathrm{HF}=-899.7593089 \backslash \mathrm{RMSD}=5.209 \mathrm{e}-09 \backslash \mathrm{RMSF}=1.052 \mathrm{e}-05 \backslash \mathrm{Dipole}=1.660995,-0.189$ $7782,0.1348838 \backslash \mathrm{PG}=\mathrm{C} 01 \quad[\mathrm{X}(\mathrm{C} 4 \mathrm{H} 13 \mathrm{P} 1 \mathrm{~S} 1)] \backslash \backslash @$

\section{For the $C=S$ Additions}

\section{$\mathrm{S}=\mathrm{C}\left(\mathrm{CH}_{3}\right) \mathrm{SCH}_{3}$}

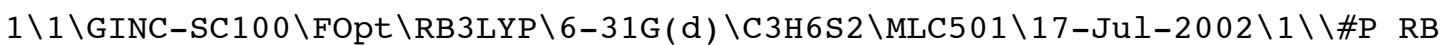
$3 \mathrm{LYP} / 6-31 \mathrm{G} *$ FOPT $=($ MAXCYC $=150, \mathrm{Z}-\mathrm{MATRIX}) \quad \mathrm{MAXDISK}=131072000$ FREQ SCF $=\mathrm{TIGH}$

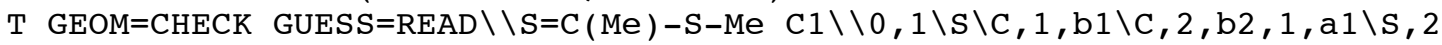
, b3 , $1, \mathrm{a} 2,3, \mathrm{dh} 3,0 \backslash \mathrm{C}, 4, \mathrm{~b} 4,2, \mathrm{a} 3,1, \mathrm{dh} 4,0 \backslash \mathrm{H}, 5, \mathrm{~b} 5,4, \mathrm{a} 4,2, \mathrm{dh} 5,0 \backslash \mathrm{H}, 5, \mathrm{~b} 6,4, \mathrm{a} 5,2$ , dh $1,0 \backslash \mathrm{H}, 5, \mathrm{~b} 6 \mathrm{a}, 4, \mathrm{a} 5 \mathrm{a}, 2,-\mathrm{dh} 1 \mathrm{a}, 0 \backslash \mathrm{H}, 3, \mathrm{~b} 7,2$, a $6,1, \mathrm{dh} 6,0 \backslash \mathrm{H}, 3, \mathrm{~b} 8,2, \mathrm{a} 7,1, \mathrm{dh} 2,0$ $\backslash \mathrm{H}, 3, \mathrm{~b} 8 \mathrm{a}, 2, \mathrm{a} 7 \mathrm{a}, 1,-\mathrm{dh} 2 \mathrm{a}, 0 \backslash \backslash \mathrm{b} 1=1.64599636 \backslash \mathrm{b} 2=1.5148461 \backslash \mathrm{b} 3=1.75511783 \backslash \mathrm{b} 4=$ $1.81794969 \backslash \mathrm{b} 5=1.09307671 \backslash \mathrm{b} 6=1.0919738 \backslash \mathrm{b} 6 \mathrm{a}=1.09197036 \backslash \mathrm{b} 7=1.09324611 \backslash \mathrm{b} 8=$ $1.09537804 \backslash b 8 a=1.0962657 \backslash a 1=122.84648556 \backslash a 2=125.75342086 \backslash a 3=103.776055$ $3 \backslash a 4=106.19073532 \backslash a 5=110.33849971 \backslash a 5 a=110.27255399 \backslash a 6=109.69980988 \backslash a 7=$ $112.81878244 \backslash a 7 a=109.03573022 \backslash \mathrm{dh} 1=60.70823437 \backslash \mathrm{dh} 1 \mathrm{a}=60.13720525 \backslash \mathrm{dh} 2=167$ $.56947484 \backslash d h 2 a=72.03339216 \backslash d h 3=181.23401229 \backslash d h 4=0.90237034 \backslash d h 5=180.305$ $77272 \backslash \mathrm{dh} 6=45.4338183 \backslash \backslash$ Version=DEC-AXP-OSF $/ 1-G 98 \mathrm{RevA} .11 .3 \backslash \mathrm{HF}=-914.30737$ $69 \backslash \mathrm{RMSD}=3.465 \mathrm{e}-10 \backslash \mathrm{RMSF}=1.676 \mathrm{e}-06 \backslash \mathrm{Dipole}=-0.3544958,-0.0417535,0.694415$ $9 \backslash \mathrm{PG}=\mathrm{C} 01 \quad[\mathrm{X}(\mathrm{C} 3 \mathrm{H} 6 \mathrm{~S} 2)] \backslash \backslash \mathrm{Q}$

\section{TS: $\cdot \mathrm{CH}_{3}+\mathrm{S}=\mathrm{C}\left(\mathrm{CH}_{3}\right) \mathrm{SCH}_{3}$}

$1 \backslash 1 \backslash G I N C-S C 70 \backslash F T S \backslash U B 3 L Y P \backslash 6-31 G(d) \backslash C 4 H 9 S 2(2) \backslash M L C 501 \backslash 17-J u 1-2002 \backslash 1 \backslash \backslash \# \mathrm{~N}$ $\mathrm{B} 3 \mathrm{LYP} / 6-31 \mathrm{G}$ (D) OPT=(TS, EF, CALCFC, Z-MATRIX, MAXCYC=100) FREQ MAXDISK=131 
$072000 \mathrm{SCF}=(\mathrm{TIGHT}, \mathrm{MAXCYC}=100) \backslash \backslash$ mescmesmeTS-C $1 \mathrm{~b}-6 \mathrm{dub} 3 \backslash \backslash 0,2 \backslash \mathrm{S} \backslash \mathrm{C}, 1, \mathrm{~B} 1 \backslash \mathrm{S}, 2$ , $\mathrm{B} 2,1, \mathrm{~A} 1 \backslash \mathrm{C}, 2, \mathrm{~B} 3,1, \mathrm{~A} 2,3, \mathrm{D} 1,0 \backslash \mathrm{C}, 3, \mathrm{~B} 4,2, \mathrm{~A} 3,1, \mathrm{D} 2,0 \backslash \mathrm{H}, 5, \mathrm{~B} 5,3, \mathrm{~A} 4,2, \mathrm{D} 3,0 \backslash \mathrm{H}, 5$, $\mathrm{B} 6,3, \mathrm{~A} 5,6, \mathrm{D} 4,0 \backslash \mathrm{H}, 5, \mathrm{~B} 7,3, \mathrm{~A} 6,6, \mathrm{D} 5,0 \backslash \mathrm{C}, 1, \mathrm{~B} 8,2, \mathrm{~A} 7,3, \mathrm{D} 6,0 \backslash \mathrm{H}, 9, \mathrm{~B} 9,1, \mathrm{~A} 8,2$, D7 , $0 \backslash \mathrm{H}, 9, \mathrm{~B} 10,1, \mathrm{~A} 9,10, \mathrm{D} 8,0 \backslash \mathrm{H}, 9, \mathrm{~B} 11,1, \mathrm{~A} 10,10, \mathrm{D} 9,0 \backslash \mathrm{H}, 4, \mathrm{~B} 12,2, \mathrm{~A} 11,1, \mathrm{D} 10,0 \backslash \mathrm{H}, 4$ , $\mathrm{B} 13,2, \mathrm{~A} 12,1, \mathrm{D} 11,0 \backslash \mathrm{H}, 4, \mathrm{~B} 14,2, \mathrm{~A} 13,1, \mathrm{D} 12,0 \backslash \backslash \mathrm{B} 1=1.65090491 \backslash \mathrm{B} 2=1.76226163 \backslash$ $\mathrm{B} 3=1.51367224 \backslash \mathrm{B} 4=1.81883498 \backslash \mathrm{B} 5=1.09315991 \backslash \mathrm{B} 6=1.09202887 \backslash \mathrm{B} 7=1.09173616 \backslash$ $\mathrm{B} 9=1.08242665 \backslash \mathrm{B} 10=1.08312635 \backslash \mathrm{B} 11=1.08306075 \backslash \mathrm{B} 12=1.09253826 \backslash \mathrm{B} 13=1.09797$ $114 \backslash \mathrm{B} 14=1.09596882 \backslash \mathrm{A} 1=125.62145537 \backslash \mathrm{A} 2=122.8978683 \backslash \mathrm{A} 3=103.82801251 \backslash \mathrm{A} 4=1$ $06.14424305 \backslash A 5=110.34937531 \backslash A 6=110.58546476 \backslash A 7=112.97083096 \backslash A 8=96.1395$ $3096 \backslash A 9=98.03637008 \backslash A 10=92.94745782 \backslash A 11=109.96652475 \backslash A 12=109.5126919 \backslash A$ $13=112.36453295 \backslash D 1=-183.88973235 \backslash D 2=-1.2889061 \backslash D 3=180.94499783 \backslash D 4=119$. $40180162 \backslash D 5=-119.54246872 \backslash D 6=-93.40650499 \backslash D 7=-86.24438242 \backslash D 8=120.62612$ $263 \backslash D 9=-119.6656341 \backslash D 10=34.13325402 \backslash D 11=-84.13835705 \backslash D 12=156.15698465 \backslash$ $\mathrm{B} 8=2.76574916 \backslash \backslash$ Version=DEC-AXP-OSF $/ 1-\mathrm{G} 98 \mathrm{RevA} .11 .3 \backslash \mathrm{HF}=-954.1441368 \backslash \mathrm{S} 2=0$ $.772159 \backslash \mathrm{S} 2-1=0 . \backslash \mathrm{S} 2 \mathrm{~A}=0.75014 \backslash \mathrm{RMSD}=8.680 \mathrm{e}-09 \backslash \mathrm{RMSF}=1.475 \mathrm{e}-05 \backslash \mathrm{Dipole}=0.278$ $789,0.2862317,0.3519356 \backslash \mathrm{PG}=\mathrm{C} 01 \quad[\mathrm{X}(\mathrm{C} 4 \mathrm{H} 9 \mathrm{~S} 2)] \backslash \backslash @$

\section{$\mathrm{CH}_{3} \mathrm{SC} \cdot\left(\mathrm{CH}_{3}\right) \mathrm{S}-\mathrm{CH}_{3}$}

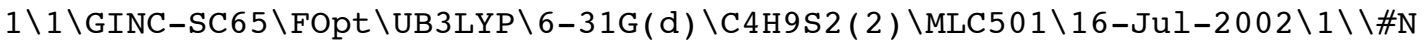
UB3LYP $/ 6-31 \mathrm{G}(\mathrm{D})$ FOPT $=(Z-M A T R I X, T I G H T, M A X C Y C=100)$ FREQ MAXDISK $=13107200$ $0 \mathrm{SCF}=(\mathrm{TIGHT}, \mathrm{MAXCYC}=100) \mathrm{GEOM}=\mathrm{CHECK}$ GUESS $=\mathrm{READ} \backslash \backslash$ mescmesme $-\mathrm{C} 1 \mathrm{~b}-6 \mathrm{dub} 3 \backslash \backslash 0$ , $2 \backslash \mathrm{H} \backslash \mathrm{C}, 1, \mathrm{~B} 1 \backslash \mathrm{H}, 2, \mathrm{~B} 2,1, \mathrm{~A} 1 \backslash \mathrm{S}, 2, \mathrm{~B} 3,1, \mathrm{~A} 2,3, \mathrm{D} 1,0 \backslash \mathrm{C}, 4, \mathrm{~B} 4,2, \mathrm{~A} 3,1, \mathrm{D} 2,0 \backslash \mathrm{S}, 5, \mathrm{~B} 5,4$ $, \mathrm{A} 4,2, \mathrm{D} 3,0 \backslash \mathrm{C}, 6, \mathrm{~B} 6,5, \mathrm{~A} 5,4,-\mathrm{D} 4,0 \backslash \mathrm{H}, 7, \mathrm{~B} 7,6, \mathrm{~A} 6,5, \mathrm{D} 5,0 \backslash \mathrm{H}, 7, \mathrm{~B} 8,6, \mathrm{~A} 7,8, \mathrm{D} 6,0 \backslash \mathrm{H}$ , $7, \mathrm{~B} 9,6, \mathrm{~A} 8,8, \mathrm{D} 7,0 \backslash \mathrm{C}, 5, \mathrm{~B} 10,4, \mathrm{~A} 9,2, \mathrm{D} 8,0 \backslash \mathrm{H}, 2, \mathrm{~B} 11,1, \mathrm{~A} 10,3, \mathrm{D} 9,0 \backslash \mathrm{H}, 11, \mathrm{~B} 12,5$, $\mathrm{A} 11,4, \mathrm{D} 10,0 \backslash \mathrm{H}, 11, \mathrm{~B} 13,5, \mathrm{~A} 12,4, \mathrm{D} 11,0 \backslash \mathrm{H}, 11, \mathrm{~B} 14,5, \mathrm{~A} 13,4, \mathrm{D} 12,0 \backslash \backslash \mathrm{B} 1=1.092657$ $93 \backslash \mathrm{B} 2=1.09285939 \backslash \mathrm{B} 3=1.83510395 \backslash \mathrm{B} 4=1.77013049 \backslash \mathrm{B} 5=1.75804982 \backslash \mathrm{B} 6=1.832220$ $08 \backslash \mathrm{B} 7=1.09257315 \backslash \mathrm{B} 8=1.09085787 \backslash \mathrm{B} 9=1.09328827 \backslash \mathrm{B} 10=1.50447696 \backslash \mathrm{B} 11=1.0934$ $6331 \backslash \mathrm{B} 12=1.09613505 \backslash \mathrm{B} 13=1.09553397 \backslash \mathrm{B} 14=1.10178689 \backslash \mathrm{A} 1=110.0297286 \backslash \mathrm{A} 2=11$ $0.82443615 \backslash A 3=102.23773298 \backslash A 4=118.51568336 \backslash A 5=103.96848806 \backslash A 6=110.4950$ $1693 \backslash A 7=111.21266523 \backslash A 8=105.91951214 \backslash A 9=120.43443778 \backslash A 10=109.33705389 \backslash$ $\mathrm{A} 11=111.15729093 \backslash \mathrm{A} 12=110.74166016 \backslash \mathrm{A} 13=112.20742397 \backslash \mathrm{D} 1=-124.06148497 \backslash \mathrm{D} 2$ $=60.56470818 \backslash \mathrm{D} 3=-144.49451711 \backslash \mathrm{D} 4=-48.977946 \backslash \mathrm{D} 5=60.52936234 \backslash \mathrm{D} 6=-122.478$ $49479 \backslash D 7=118.55770463 \backslash D 8=62.40591497 \backslash D 9=119.28641302 \backslash D 10=-44.11186517 \backslash$ $\mathrm{D} 11=-164.00422423 \backslash \mathrm{D} 12=75.46393503 \backslash \backslash$ Version=DEC-AXP-OSF / 1-G98RevA.11.3\ $\mathrm{HF}=-954.1760596 \backslash \mathrm{S} 2=0.755169 \backslash \mathrm{S} 2-1=0 . \backslash \mathrm{S} 2 \mathrm{~A}=0.75002 \backslash \mathrm{RMSD}=5.132 \mathrm{e}-09 \backslash \mathrm{RMSF}=2$. $815 e-05 \backslash \mathrm{Dipole}=0.2098476,0.5512682,-0.4657697 \backslash \mathrm{PG}=\mathrm{C} 01 \quad[\mathrm{X}(\mathrm{C} 4 \mathrm{H} 9 \mathrm{~S} 2)] \backslash \backslash @$

\section{$\mathrm{CH}_{3} \mathrm{SCH}\left(\mathrm{CH}_{3}\right) \mathrm{S}-\mathrm{CH}_{3}$}

$1 \backslash 1 \backslash G I N C-S C 50 \backslash F O p t \backslash R B 3 L Y P \backslash 6-31 G(d) \backslash C 4 H 10 S 2 \backslash M L C 501 \backslash 16-J a n-2003 \backslash 0 \backslash \backslash \# N$ B3

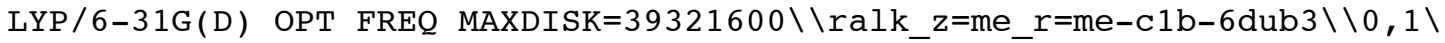
$\mathrm{H},-0.8520756361,0.8033167342,2.6879932569 \backslash \mathrm{C}, \overline{0} .237 \overline{0} 28745,0.7400719558,2$ $.5967512165 \backslash \mathrm{H}, 0.66012251,1.7461819529,2.5241712482 \backslash \mathrm{S}, 0.7265755304,-0.3$ $135602287,1.182376267 \backslash \mathrm{C},-0.0981477987,0.5907986636,-0.2087141703 \backslash \mathrm{S},-0$. $2432081378,-0.5178018206,-1.6643786617 \backslash \mathrm{C},-1.5184006495,-1.6897698027,-$ $1.08384029 \backslash \mathrm{H},-2.4735833353,-1.1832268548,-0.9147693289 \backslash \mathrm{H},-1.1948363437$ $,-2.1931652975,-0.1698746482 \backslash \mathrm{H},-1.6462270616,-2.4302742312,-1.87782798$ $13 \backslash \mathrm{C}, 0.6612927142,1.8492910624,-0.6303946624 \backslash \mathrm{H}, 0.6349852607,0.26071070$ $74,3.4953485823 \backslash \mathrm{H}, 0.7958825981,2.5207757598,0.2239981057 \backslash \mathrm{H}, 0.107849916$ $6,2.3888619535,-1.407314693 \backslash \mathrm{H}, 1.6524532115,1.5937434843,-1.0176689327 \backslash$ $\mathrm{H},-1.1090874676,0.8525173058,0.1251701442 \backslash \backslash$ Version=DEC-AXP-OSF $/ 1-G 98 \mathrm{Re}$ $\mathrm{VA} .11 .3 \backslash \mathrm{HF}=-954.8250473 \backslash \mathrm{RMSD}=5.044 \mathrm{e}-09 \backslash \mathrm{RMSF}=1.205 \mathrm{e}-05 \backslash \mathrm{Dipole}=-0.682714$ $7,0.4700445,0.5466841 \backslash \mathrm{PG}=\mathrm{C} 01 \quad[\mathrm{X}(\mathrm{C} 4 \mathrm{H} 10 \mathrm{~S} 2)] \backslash \backslash @$

\section{$\mathrm{S}=\mathrm{C}\left(\mathrm{OCH}_{3}\right) \mathrm{SCH}_{3}$}

$1 \backslash 1 \backslash G I N C-S C 160 \backslash F O p t \backslash R B 3 L Y P \backslash 6-31 G(d) \backslash C 3 H 601 S 2 \backslash M L C 501 \backslash 28-A p r-2003 \backslash 0 \backslash \backslash \# N$ B3LYP $/ 6-31 \mathrm{G}$ (D) OPT= (TIGHT, MAXCYC=100) FREQ MAXDISK=131072000 GEOM=CHEC $\mathrm{K}$ GUESS $=\mathrm{READ} \backslash \backslash \mathrm{RAFT}(\mathrm{Z}=\mathrm{OMe} \mathrm{R}=\mathrm{CH} 3)$ lowest $\mathrm{a} 1 \mathrm{~b} 1$ in $\mathrm{Cs}$ symm $\backslash \backslash 0,1 \backslash \mathrm{C}, 0.374209$ $3441,-2.4921458868,0 . \backslash S, 1.301426637,-0.9260065359,0 . \backslash \mathrm{C},-0.0000019362,0$ $.2785978098,0 . \backslash \mathrm{S},-1.6256039629,-0.0059529119,0 . \backslash 0,0.5982011851,1.47426$ $53379,0 . \backslash \mathrm{C},-0.2142814517,2.6603482395,0 . \backslash \mathrm{H}, 1.1323627109,-3.280322659,0$ $. \backslash \mathrm{H},-0.2490760809,-2.5725262915,0.8922739391 \backslash \mathrm{H},-0.2490760809,-2.572526$ $2915,-0.8922739391 \backslash \mathrm{H}, 0.4987378961,3.485149722,0 . \backslash \mathrm{H},-0.8456382241,2.688$ 
$326503,-0.8916364756 \backslash \mathrm{H},-0.8456382241,2.688326503,0.8916364756 \backslash \backslash$ Version =DEC-AXP-OSF / 1-G98RevA.11.3 \State $=1-A^{\prime} \backslash \mathrm{HF}=-989.5242355 \backslash$ RMSD $=5.328 \mathrm{e}-09 \backslash$ $\mathrm{RMSF}=3.625 \mathrm{e}-06 \backslash \mathrm{Dipole}=0.2790653,0.1622872,0 . \backslash \mathrm{PG}=\mathrm{CS}[\mathrm{SG}(\mathrm{C} 3 \mathrm{H} 2 \mathrm{O} 1 \mathrm{~S} 2), \mathrm{X}(\mathrm{H} 4)$ ]$\backslash \backslash @$

\section{TS: $\cdot \mathrm{CH}_{3}+\mathrm{S}=\mathrm{C}\left(\mathrm{OCH}_{3}\right) \mathrm{SCH}_{3}$}

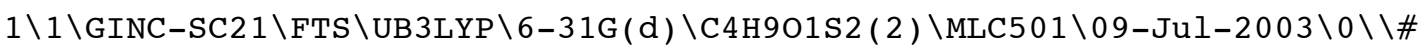
B3LYP /6-31G* OPT=(TS,CALCFC, NOEIGENTEST, MAXCYC=100) FREQ MAXDISK=52428 800 INT (GRID=ULTRAFINE) IOP $(1 / 8=5) \backslash \backslash \mathrm{TS}$ Me+RAFT_Z=OMe_R=CH3-new3-6dub3 $\backslash 0,2 \backslash S,-1.3072338583,-0.2307872537,-0 . \overline{2} 1236151 \overline{7} 5 \backslash \mathrm{C}, 0.3485347442,-0.249$ $2551772,-0.3156473865 \backslash \mathrm{S}, 1.4411184265,1.1510297031,-0.3488296927 \backslash \mathrm{C}, 0.27$ $69144349,2.5492107131,-0.4136663031 \backslash C,-2.1507732279,-0.1800884538,2.15$ $86997117 \backslash 0,1.1392367475,-1.3415421381,-0.3114255047 \backslash \mathrm{C}, 0.5287284685,-2$. $6372303303,-0.3114353021 \backslash \mathrm{H},-1.3532110571,0.3678573025,2.6487039331 \backslash \mathrm{H},-$ $3.0728685448,0.3570541837,1.9632657307 \backslash \mathrm{H},-2.2288431901,-1.2404787643,2$ $.3709420912 \backslash \mathrm{H},-0.3521954105,2.4896613409,-1.3037983338 \backslash \mathrm{H}, 0.9002932184$, $3.446702728,-0.4586819363 \backslash \mathrm{H},-0.3513312889,2.5786500685,0.4781445138 \backslash \mathrm{H}$, $1.3619311765,-3.3409057237,-0.3325199709 \backslash \mathrm{H},-0.0738908465,-2.7814941223$ $, 0.5896866552 \backslash \mathrm{H},-0.1063576464,-2.7644096105,-1.1929836032 \backslash \backslash$ Version=DEC $-\mathrm{AXP}-\mathrm{OSF} / 1-\mathrm{G} 03 \mathrm{RevB} .03 \backslash \mathrm{State}=2-\mathrm{A} \backslash \mathrm{HF}=-1029.3567056 \backslash \mathrm{S} 2=0.773045 \backslash \mathrm{S} 2-1=0 . \backslash \mathrm{S}$ $2 A=0.750123 \backslash R M S D=6.891 e-09 \backslash R M S F=6.727 e-06 \backslash D i p o l e=-0.0737059,-0.1057122$ , $0.2070148 \backslash \mathrm{PG}=\mathrm{C} 01[\mathrm{X}(\mathrm{C} 4 \mathrm{H} 901 \mathrm{~S} 2)] \backslash \backslash @$

\section{$\mathrm{CH}_{3} \mathrm{SC} \cdot\left(\mathrm{OCH}_{3}\right) \mathrm{SCH}_{3}$}

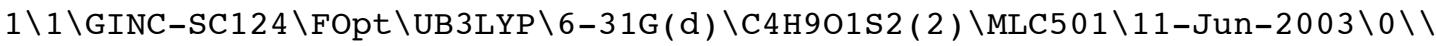
\# B3LYP $/ 6-31 \mathrm{G} * \mathrm{OPT}=(\mathrm{MAXCYC}=100)$ FREQ MAXDISK $=13107200$ GEOM=CHECK GUESS $=$ READ \\rrad $z=0$ e $r=m e-a 1 b 1 c 1 d 3 e 2 f 1-6$ dub3 $\backslash \backslash 0,2 \backslash S,-1.3410354108,-1.1021$ $645587,0.00837807 \overline{2} 2 \backslash \mathrm{C},-0.0668314555,0.0846927765,-0.2625382649 \backslash \mathrm{S}, 1.525$ $0665723,-0.5327357432,-0.7113926887 \backslash \mathrm{C}, 2.3426793192,-0.7855867589,0.929$ $7015005 \backslash \mathrm{C},-2.8220656021,-0.0301869566,0.1187230656 \backslash 0,-0.1829188202,1.2$ $334385003,0.4787862469 \backslash \mathrm{C}, 0.3588901181,2.4188570239,-0.1145681207 \backslash \mathrm{H},-2$. $9779210372,0.5082852285,-0.8196451416 \backslash \mathrm{H},-3.670068575,-0.6955390127,0.2$ $998754487 \backslash \mathrm{H},-2.72432788,0.675718492,0.9443720255 \backslash \mathrm{H}, 2.2450048802,0.1147$ $964264,1.5398219577 \backslash \mathrm{H}, 3.400214885,-0.9780596806,0.7296543233 \backslash \mathrm{H}, 1.90496$ $92578,-1.6399132978,1.4510056405 \backslash \mathrm{H}, 0.1716693923,3.2223284691,0.6006696$ $643 \backslash \mathrm{H}, 1.4348670988,2.3181216146,-0.2907133132 \backslash \mathrm{H},-0.1415903241,2.638502$ $0801,-1.0650057993 \backslash \backslash$ Version=DEC-AXP-OSF $/ 1-$ G03RevB .03 $\backslash$ State $=2-A \backslash H F=-102$ $9.3786222 \backslash \mathrm{S} 2=0.754676 \backslash \mathrm{S} 2-1=0 . \backslash \mathrm{S} 2 \mathrm{~A}=0.750016 \backslash \mathrm{RMSD}=3.068 \mathrm{e}-09 \backslash \mathrm{RMSF}=1.311 \mathrm{e}-$ $06 \backslash \mathrm{Dipole}=-0.1181936,0.8010544,0.3889717 \backslash \mathrm{PG}=\mathrm{C} 01 \quad[\mathrm{X}(\mathrm{C} 4 \mathrm{H} 901 \mathrm{~S} 2)] \backslash \backslash @$

\section{$\mathrm{CH}_{3} \mathrm{SCH}\left(\mathrm{OCH}_{3}\right) \mathrm{SCH}_{3}$}

$1 \backslash 1 \backslash \mathrm{GINC}-\mathrm{SC} 160 \backslash \mathrm{FOpt} \backslash \mathrm{RB} 3 \mathrm{LYP} \backslash 6-31 \mathrm{G}(\mathrm{d}) \backslash \mathrm{C} 4 \mathrm{H} 1001 \mathrm{~S} 2 \backslash \mathrm{MLC} 501 \backslash 13-\mathrm{Aug}-2003 \backslash 0 \backslash \backslash \#$ B3LYP $/ 6-31 \mathrm{G} *$ OPT $=($ MAXCYC $=100$, TIGHT $)$ FREQ GEOM=CHECK GUESS=READ MAXDISK

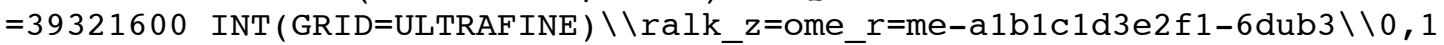
$\backslash S,-1.2638080102,-1.0946066938,-0.0388623 \overline{5} 7 \backslash \mathrm{C},-0.0661509511,0.22262651$ $51,-0.4773860228 \backslash \mathrm{S}, 1.6208062742,-0.506946341,-0.6859074301 \backslash \mathrm{C}, 1.9762100$ $951,-1.0009324492,1.0416521861 \backslash \mathrm{C},-2.7837706451,-0.0914568117,0.1427433$ $606 \backslash 0,-0.1350263577,1.2360458069,0.4876130868 \backslash \mathrm{C}, 0.4025768371,2.4839329$ $534,0.0588080284 \backslash \mathrm{H},-3.067093543,0.3769686088,-0.804977898 \backslash \mathrm{H},-3.5760666$ $241,-0.7816445842,0.4449633038 \backslash \mathrm{H},-2.649622786,0.6701274942,0.912327733$ $8 \backslash \mathrm{H}, 1.6788946359,-0.1976058747,1.7188468452 \backslash \mathrm{H}, 3.0563328618,-1.15320241$ $87,1.1078188609 \backslash \mathrm{H}, 1.458985461,-1.9239787154,1.3096252981 \backslash \mathrm{H}, 0.253256020$ $9,3.1821978333,0.8850644167 \backslash \mathrm{H}, 1.4725885627,2.4014309461,-0.1672236522 \backslash$ $\mathrm{H},-0.1241541813,2.8566842465,-0.8321509639 \backslash \mathrm{H},-0.3080737866,0.620483318$ $7,-1.473787359 \backslash \backslash$ Version=DEC-AXP-OSF $/ 1-G 03$ RevB . 03 $\backslash$ State $=1-A \backslash H F=-1030.02$ $74547 \backslash \mathrm{RMSD}=3.600 \mathrm{e}-09 \backslash \mathrm{RMSF}=3.094 \mathrm{e}-07 \backslash \mathrm{Dipole}=-0.2244423,0.8750638,0.1944$ $09 \backslash P G=C 01 \quad[X(C 4 H 1001 S 2)] \backslash \backslash @$

\section{$\mathrm{S}=\mathrm{C}(\mathrm{CN}) \mathrm{SCH}_{3}$}

$1 \backslash 1 \backslash G I N C-S C 126 \backslash F O p t \backslash R B 3 L Y P \backslash 6-31 G(d) \backslash C 3 H 3 N 1 S 2 \backslash D J H 501 \backslash 10-M a y-2004 \backslash 0 \backslash \backslash \# B$ $3 \mathrm{LYP} / 6-31 \mathrm{G} * \mathrm{SCF}=\mathrm{TIGHT}$ OPT=TIGHT MAXDISK=39321600 IOP $(1 / 8=10) \backslash \backslash \mathrm{CH} 3 \mathrm{SC}(\mathrm{CN}$ ) Sb B3LYP / 6-31G(d) geom opt $\backslash \backslash 0,1 \backslash C, 1.7143130431,-0.4903533238,-0.000$ 
$0026937 \backslash \mathrm{C}, 0.405329302,0.1041091147,-0.0000016782 \backslash \mathrm{S},-0.8432374325,-1.12$ $90645757,0.0000193385 \backslash \mathrm{C},-2.3647846875,-0.1341256382,0.0000040713 \backslash \mathrm{S}, 0.2$ $21723625,1.7406005545,-0.0000181688 \backslash \mathrm{H},-3.1883355575,-0.8523386291,-0.0$ $000190697 \backslash \mathrm{H},-2.4091215943,0.4936780571,0.8925537675 \backslash \mathrm{H},-2.4090883069,0$. $4936981649,-0.8925330863 \backslash \mathrm{N}, 2.7745172047,-0.9710563101,-0.0000026461 \backslash \backslash \mathrm{V}$ ersion=DEC-AXP-OSF $/ 1-G 03 R e v B .05 \backslash$ State $=1-A \backslash H F=-967.2190127 \backslash$ RMSD $=7.862 e-$ $09 \backslash \mathrm{RMSF}=2.473 e-06 \backslash \mathrm{Dipole}=-1.8143545,-0.0479982,0.0000052 \backslash \mathrm{PG}=\mathrm{C} 01 \quad[\mathrm{X}(\mathrm{C} 3 \mathrm{H}$ $3 \mathrm{~N} 1 \mathrm{~S} 2)] \backslash \backslash @$

\section{$\mathrm{CH}_{3} \mathrm{SC} \cdot(\mathrm{CN}) \mathrm{SCH}_{3}$}

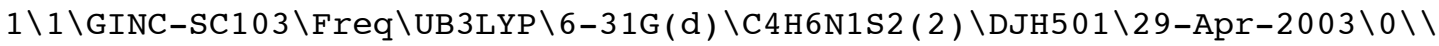
\#N UB3LYP/6-31G(D) SCF=TIGHT FREQ MAXDISK=39321600\\CH3SC(CN)SCH3d rad

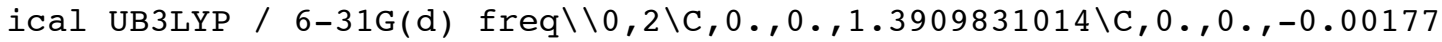
$88986 \backslash \mathrm{S}, 0 ., 1.48737,-0.9364158986 \backslash \mathrm{C},-0.95775,2.619045,0.1386721014 \backslash \mathrm{S}, 0$. $,-1.48737,-0.9364158986 \backslash \mathrm{C}, 0.95775,-2.619045,0.1386721014 \backslash \mathrm{H},-1.992213,2$ $.280518,0.2285301014 \backslash \mathrm{H},-0.499302,2.697444,1.1267991014 \backslash \mathrm{H},-0.93062,3.59$ $448,-0.3533868986 \backslash \mathrm{H}, 1.992213,-2.280518,0.2285301014 \backslash \mathrm{H}, 0.499302,-2.6974$ $44,1.1267991014 \backslash \mathrm{H}, 0.93062,-3.59448,-0.3533868986 \backslash \mathrm{N}, 0 ., 0 ., 2.5660191014 \backslash$ $\backslash$ Version=DEC-AXP-OSF $/ 1-G 98 R e v A .11 .3 \backslash$ State $=2-B \backslash H F=-1007.1120674 \backslash \mathrm{S} 2=0.75$ $9884 \backslash \mathrm{S} 2-1=0 . \backslash \mathrm{S} 2 \mathrm{~A}=0.750062 \backslash \mathrm{RMSD}=6.107 \mathrm{e}-09 \backslash \mathrm{RMSF}=4.470 \mathrm{e}-07 \backslash \mathrm{Dipole}=0 ., 0 .,-$ $0.6530448 \backslash \mathrm{PG}=\mathrm{C} 02[\mathrm{C} 2(\mathrm{C} 1 \mathrm{C} 1 \mathrm{~N} 1), \mathrm{X}(\mathrm{C} 2 \mathrm{H} 6 \mathrm{~S} 2)] \backslash \backslash @$

\section{$\mathrm{CH}_{3} \mathrm{SCH}(\mathrm{CN}) \mathrm{SCH}_{3}$}

$1 \backslash 1 \backslash G I N C-S C 160 \backslash F O p t \backslash R B 3 L Y P \backslash 6-31 G(d) \backslash C 4 H 7 N 1 S 2 \backslash D J H 501 \backslash 27-J u n-2004 \backslash 0 \backslash \backslash \# N$ B3LYP/6-31G* SCF=TIGHT OPT MAXDISK=39321600 \\CH3SCH(CN)SCH3d B3LYP / 6 $-31 G(d) \backslash \backslash 0,1 \backslash C, 0.2156111309,-0.3806481937,0.5726710689 \backslash C, 1.6749493252$, $-0.3723893247,0.5237508527 \backslash S,-0.3514477731,1.3678645895,0.8129334278 \backslash S$ $,-0.4944245279,-1.2902365825,-0.8732613703 \backslash \mathrm{C},-2.1059866343,1.047783498$ $2,1.201115454 \backslash \mathrm{C}, 0.2317869576,-0.3442074892,-2.2564143791 \backslash \mathrm{H},-0.10447469$ $19,-0.9684150507,1.4395252678 \backslash \mathrm{N}, 2.8333574203,-0.3557759993,0.439583949$ $6 \backslash \mathrm{H},-2.5826133741,0.4858173859,0.3940373775 \backslash \mathrm{H},-2.2131266262,0.51349706$ $21,2.1498771422 \backslash \mathrm{H},-2.5834253016,2.0271112795,1.2890856843 \backslash \mathrm{H}, 1.32029221$ $64,-0.4413767331,-2.2657773305 \backslash \mathrm{H},-0.0558635201,0.7082115292,-2.1943696$ $316 \backslash \mathrm{H},-0.1784985043,-0.7796925329,-3.1709570557 \backslash \backslash$ Version=DEC-AXP-OSF $/ 1$ -G03RevB. 05 \State $=1-A \backslash H F=-1007.7426907 \backslash R M S D=3.851 e-09 \backslash$ RMSF $=2.199 e-05 \backslash D$ ipole $=-1.5272915,0.0786747,-0.12118 \backslash \mathrm{PG}=\mathrm{C} 01[\mathrm{X}(\mathrm{C} 4 \mathrm{H} 7 \mathrm{~N} 1 \mathrm{~S} 2)] \backslash \backslash @$

\section{$\mathrm{S}=\mathrm{C}(\mathrm{Ph}) \mathrm{SCH}_{3}$}

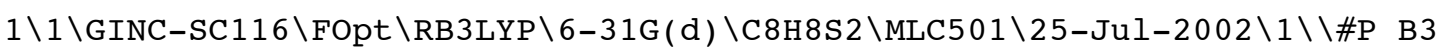
LYP /6-31G* OPT $=(Z-M A T R I X, M A X C Y C=100)$ FREQ MAXDISK $=131072000 \backslash \backslash$ RAFT agen t $\mathrm{S}=\mathrm{C}(\mathrm{Ph})-\mathrm{S}-\mathrm{CH} 3 \quad(\mathrm{Z}=\mathrm{ph}, \mathrm{R}=\mathrm{me}) \backslash \backslash 0,1 \backslash \mathrm{C} \backslash \mathrm{S}, 1, \mathrm{~B} 1 \backslash \mathrm{S}, 1, \mathrm{~B} 2,2, \mathrm{~A} 1 \backslash \mathrm{C}, 3, \mathrm{~B} 3,1, \mathrm{~A} 2,2, \mathrm{D} 1$ $, 0 \backslash \mathrm{H}, 4, \mathrm{~B} 4,3, \mathrm{~A} 3,1, \mathrm{D} 2,0 \backslash \mathrm{H}, 4, \mathrm{~B} 5,3, \mathrm{~A} 4,5, \mathrm{D} 3,0 \backslash \mathrm{H}, 4, \mathrm{~B} 6,3, \mathrm{~A} 5,5, \mathrm{D} 4,0 \backslash \mathrm{C}, 1, \mathrm{~B} 7,2, \mathrm{~A}$ $6,3, \mathrm{D} 5,0 \backslash \mathrm{C}, 8, \mathrm{~B} 8,1, \mathrm{~A} 7,2, \mathrm{D} 6,0 \backslash \mathrm{C}, 9, \mathrm{~B} 9,8, \mathrm{~A} 8,1, \mathrm{D} 7,0 \backslash \mathrm{C}, 10, \mathrm{~B} 10,9, \mathrm{~A} 9,8, \mathrm{D} 8,0 \backslash \mathrm{C}$, $11, \mathrm{~B} 11,10, \mathrm{~A} 10,9, \mathrm{D} 9,0 \backslash \mathrm{C}, 12, \mathrm{~B} 12,11, \mathrm{~A} 11,10, \mathrm{D} 10,0 \backslash \mathrm{H}, 13, \mathrm{~B} 13,12, \mathrm{~A} 12,11, \mathrm{D} 11,0$ $\backslash \mathrm{H}, 12, \mathrm{~B} 14,11, \mathrm{~A} 13,13, \mathrm{D} 12,0 \backslash \mathrm{H}, 11, \mathrm{~B} 15,10, \mathrm{~A} 14,12, \mathrm{D} 13,0 \backslash \mathrm{H}, 10, \mathrm{~B} 16,9, \mathrm{~A} 15,11, \mathrm{D}$ $14,0 \backslash \mathrm{H}, 9, \mathrm{~B} 17,8, \mathrm{~A} 16,10, \mathrm{D} 15,0 \backslash \backslash \mathrm{B} 1=1.65390665 \backslash \mathrm{B} 2=1.76783874 \backslash \mathrm{B} 3=1.81735533$ $\backslash \mathrm{B} 4=1.09340644 \backslash \mathrm{B} 5=1.09203382 \backslash \mathrm{B} 6=1.09167768 \backslash \mathrm{B} 7=1.48638437 \backslash \mathrm{B} 8=1.40791986$ $\backslash \mathrm{B} 9=1.3934782 \backslash \mathrm{B} 10=1.3954032 \backslash \mathrm{B} 11=1.39756856 \backslash \mathrm{B} 12=1.39093249 \backslash \mathrm{B} 13=1.084558$ $18 \backslash \mathrm{B} 14=1.08646979 \backslash \mathrm{B} 15=1.08663117 \backslash \mathrm{B} 16=1.08643383 \backslash \mathrm{B} 17=1.08519217 \backslash \mathrm{A} 1=123$. $8831348 \backslash A 2=103.44785578 \backslash A 3=105.74311227 \backslash A 4=110.56367906 \backslash A 5=110.5050450$ $1 \backslash A 6=123.43517028 \backslash A 7=121.7784707 \backslash A 8=120.59384832 \backslash A 9=120.24074703 \backslash A 10=1$ $19.68786961 \backslash \mathrm{A} 11=120.27839448 \backslash \mathrm{A} 12=120.55619114 \backslash \mathrm{A} 13=120.1204252 \backslash \mathrm{A} 14=120$. $14506072 \backslash A 15=119.5622211 \backslash A 16=119.91357919 \backslash D 1=5.07451867 \backslash D 2=178.7658797$ $5 \backslash D 3=-119.43094734 \backslash D 4=119.41965567 \backslash D 5=181.27901545 \backslash D 6=211.48944504 \backslash D 7=$ $179.74862327 \backslash D 8=0.5087189 \backslash D 9=-0.47334322 \backslash D 10=-0.1123593 \backslash D 11=180.118421$ $67 \backslash \mathrm{D} 12=179.95923577 \backslash \mathrm{D} 13=180.24754199 \backslash \mathrm{D} 14=180.47358662 \backslash \mathrm{D} 15=182.12059766$ $\backslash \backslash$ Version=DEC-AXP-OSF / 1-G98RevA. 11.3\HF=-1106.0452545 $\backslash \mathrm{RMSD}=3.885 \mathrm{e}-09 \backslash \mathrm{R}$ $\mathrm{MSF}=1.915 \mathrm{e}-05 \backslash \mathrm{Dipole}=0.0516049,-0.0290079,-0.8060901 \backslash \mathrm{PG}=\mathrm{C} 01 \quad[\mathrm{X}(\mathrm{C} 8 \mathrm{H} 8 \mathrm{~S} 2)$ ]$\backslash \backslash[$

\section{TS: $\cdot \mathrm{CH}_{3}+\mathrm{S}=\mathrm{C}(\mathrm{Ph}) \mathrm{SCH}_{3}$}

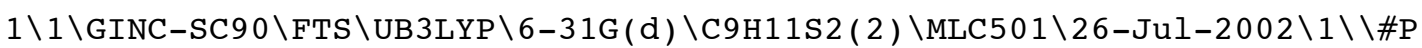


B3LYP / 6-31G* OPT= (TS, Z-MATRIX, MAXCYC $=100$, CALCFC , NOEIGENTEST $)$ MAXDISK=1 $31072000 \mathrm{SCF}=(\mathrm{QC}, \mathrm{MAXCYC}=100) \mathrm{FREQ} \backslash \backslash \mathrm{TS}$ for $\mathrm{CH} 3+\mathrm{RAFT}$ agent $\mathrm{S}=\mathrm{C}(\mathrm{Ph})-\mathrm{S}-\mathrm{CH}$ 3 ( TS: $\mathrm{Z}=\mathrm{ph}, \mathrm{R}=\mathrm{me})(\mathrm{a}) \backslash \backslash 0,2 \backslash \mathrm{C} \backslash \mathrm{S}, 1, \mathrm{~B} 1 \backslash \mathrm{S}, 1, \mathrm{~B} 2,2, \mathrm{~A} 1 \backslash \mathrm{C}, 3, \mathrm{~B} 3,1, \mathrm{~A} 2,2, \mathrm{D} 1,0 \backslash \mathrm{H}, 4$, $\mathrm{B} 4,3, \mathrm{~A} 3,1, \mathrm{D} 2,0 \backslash \mathrm{H}, 4, \mathrm{~B} 5,3, \mathrm{~A} 4,5, \mathrm{D} 3,0 \backslash \mathrm{H}, 4, \mathrm{~B} 6,3, \mathrm{~A} 5,5, \mathrm{D} 4,0 \backslash \mathrm{C}, 1, \mathrm{~B} 7,2, \mathrm{~A} 6,3$, D5 , $0 \backslash \mathrm{C}, 8, \mathrm{~B} 8,1, \mathrm{~A} 7,2, \mathrm{D} 6,0 \backslash \mathrm{C}, 9, \mathrm{~B} 9,8, \mathrm{~A} 8,1, \mathrm{D} 7,0 \backslash \mathrm{C}, 10, \mathrm{~B} 10,9, \mathrm{~A} 9,8, \mathrm{D} 8,0 \backslash \mathrm{C}, 11, \mathrm{~B} 11$, $10, \mathrm{~A} 10,9, \mathrm{D} 9,0 \backslash \mathrm{C}, 12, \mathrm{~B} 12,11, \mathrm{~A} 11,10, \mathrm{D} 10,0 \backslash \mathrm{H}, 13, \mathrm{~B} 13,12, \mathrm{~A} 12,11, \mathrm{D} 11,0 \backslash \mathrm{H}, 12, \mathrm{~B}$ $14,11, \mathrm{~A} 13,13, \mathrm{D} 12,0 \backslash \mathrm{H}, 11, \mathrm{~B} 15,10, \mathrm{~A} 14,12, \mathrm{D} 13,0 \backslash \mathrm{H}, 10, \mathrm{~B} 16,9, \mathrm{~A} 15,11, \mathrm{D} 14,0 \backslash \mathrm{H}$, $9, \mathrm{~B} 17,8, \mathrm{~A} 16,10, \mathrm{D} 15,0 \backslash \mathrm{C}, 2$, $\mathrm{B} 18,1, \mathrm{~A} 17,3, \mathrm{D} 16,0 \backslash \mathrm{H}, 19, \mathrm{~B} 19,2, \mathrm{~A} 18,1, \mathrm{D} 17,0 \backslash \mathrm{H}, 19$ , $\mathrm{B} 20,2, \mathrm{~A} 19,1, \mathrm{D} 18,0 \backslash \mathrm{H}, 19, \mathrm{~B} 21,2, \mathrm{~A} 20,1$, D19, $0 \backslash \backslash \mathrm{B} 18=2.85785244 \backslash \mathrm{A} 17=112.8854$ $7308 \backslash \mathrm{D} 16=87.54234418 \backslash \mathrm{B} 19=1.08281955 \backslash \mathrm{B} 20=1.08254509 \backslash \mathrm{B} 21=1.08254817 \backslash \mathrm{A} 18=$ $96.34150121 \backslash A 19=93.33447677 \backslash A 20=94.2496342 \backslash D 17=-10.5489024 \backslash D 18=-130.55$ $97207 \backslash \mathrm{D} 19=109.66341591 \backslash \mathrm{B} 1=1.65766937 \backslash \mathrm{B} 2=1.77315841 \backslash \mathrm{B} 3=1.81803301 \backslash \mathrm{B} 4=1$. $09350034 \backslash \mathrm{B} 5=1.09209587 \backslash \mathrm{B} 6=1.09142411 \backslash \mathrm{B} 7=1.48423492 \backslash \mathrm{B} 8=1.40872449 \backslash \mathrm{B} 9=1$. $3934021 \backslash \mathrm{B} 10=1.39544748 \backslash \mathrm{B} 11=1.39759958 \backslash \mathrm{B} 12=1.39092175 \backslash \mathrm{B} 13=1.08452426 \backslash \mathrm{B} 1$ $4=1.08664308 \backslash B 15=1.0866489 \backslash B 16=1.08655345 \backslash B 17=1.08514617 \backslash A 1=123.660962$ $99 \backslash \mathrm{A} 2=103.49634073 \backslash \mathrm{A} 3=105.71669512 \backslash \mathrm{A} 4=110.60606647 \backslash \mathrm{A} 5=110.70344078 \backslash \mathrm{A} 6=$ $123.44769264 \backslash A 7=121.77261104 \backslash A 8=120.70458187 \backslash A 9=120.28905352 \backslash A 10=119.5$ $8591948 \backslash A 11=120.32573329 \backslash A 12=120.46930469 \backslash A 13=120.08894967 \backslash A 14=120.202$ $94711 \backslash \mathrm{A} 15=119.53022621 \backslash \mathrm{A} 16=119.83190111 \backslash \mathrm{D} 1=7.65849732 \backslash \mathrm{D} 2=177.95770467 \backslash$ $\mathrm{D} 3=-119.30931244 \backslash \mathrm{D} 4=119.36346225 \backslash \mathrm{D} 5=182.6028638 \backslash \mathrm{D} 6=209.56180499 \backslash \mathrm{D} 7=179$ $.85020311 \backslash D 8=0.41205611 \backslash D 9=-0.43641868 \backslash D 10=-0.05221285 \backslash D 11=180.0617337$ $2 \backslash D 12=179.91322301 \backslash D 13=180.26246934 \backslash D 14=180.47708809 \backslash D 15=181.99245773 \backslash$ $\backslash$ Version=DEC-AXP-OSF $/ 1-G 98 R e v A .11 .3 \backslash \mathrm{HF}=-1145.8826857 \backslash \mathrm{S} 2=0.77023 \backslash \mathrm{S} 2-1=0$ $. \backslash \mathrm{S} 2 \mathrm{~A}=0.75015 \backslash \mathrm{RMSD}=0.000 \mathrm{e}+00 \backslash \mathrm{RMSF}=2.289 \mathrm{e}-05 \backslash \mathrm{Dipole}=0.0939665,0.1867077$ ,$-0.4556698 \backslash \mathrm{PG}=\mathrm{C} 01 \quad[\mathrm{X}(\mathrm{C} 9 \mathrm{H} 11 \mathrm{~S} 2)] \backslash \backslash @$

\section{$\mathrm{CH}_{3} \mathrm{SC} \cdot(\mathrm{Ph}) \mathrm{S}-\mathrm{CH}_{3}$}

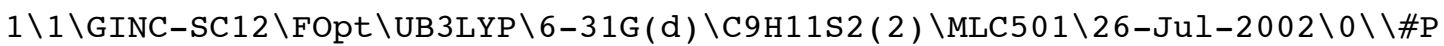
$\mathrm{B} 3 \mathrm{LYP} / 6-31 \mathrm{G} * \mathrm{OPT}=(\mathrm{MAXCYC}=100) \mathrm{SCF}=(\mathrm{MAXCYC}=100)$ FREQ MAXDISK $=131072000$ $\backslash \backslash \mathrm{rrad} z=\mathrm{ph} r=\mathrm{me}-\mathrm{c} 1 \mathrm{c}-6 \mathrm{duhf}--\mathrm{C} 2 \backslash \backslash 0,2 \backslash \mathrm{C}, 0,0,0,-0.5436243969 \backslash \mathrm{C}, 0 ., 0 ., 0.89$ $993655 \overline{5} 8 \backslash \mathrm{C}, \overline{1} .2045767983,-0.1407684102,1.6447473811 \backslash \mathrm{C},-1.2045767983,0.1$ $407684102,1.6447473811 \backslash \mathrm{C}, 1.1980023223,-0.1372926446,3.0327870849 \backslash \mathrm{C},-1$. $1980023223,0.1372926446,3.0327870849 \backslash \mathrm{H}, 2.1407212827,-0.2659311883,1.11$ $24152303 \backslash \mathrm{H},-2.1407212827,0.2659311883,1.1124152303 \backslash \mathrm{H}, 2.1367874102,-0.2$ $51386417,3.5689285696 \backslash \mathrm{H},-2.1367874102,0.251386417,3.5689285696 \backslash \mathrm{C}, 0 ., 0$.

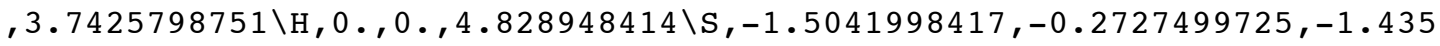
$6947229 \backslash \mathrm{C},-1.5483731257,1.1133863252,-2.639460946 \backslash \mathrm{H},-2.4327150559,0.94$ $51928411,-3.2601525901 \backslash \mathrm{H},-1.6417073267,2.0701943282,-2.120752452 \backslash \mathrm{H},-0$. $656494302,1.1210366726,-3.2689146201 \backslash S, 1.5041998417,0.2727499725,-1.43$ $56947229 \backslash \mathrm{C}, 1.5483731257,-1.1133863252,-2.639460946 \backslash \mathrm{H}, 2.4327150559,-0.9$ $451928411,-3.2601525901 \backslash \mathrm{H}, 1.6417073267,-2.0701943282,-2.120752452 \backslash \mathrm{H}, 0$. $656494302,-1.1210366726,-3.2689146201 \backslash \backslash$ Version=DEC-AXP-OSF / 1-G98RevA . 1 $1.3 \backslash$ State $=2-B \backslash H F=-1145.9239982 \backslash S 2=0.772251 \backslash \mathrm{S} 2-1=0 . \backslash \mathrm{S} 2 \mathrm{~A}=0.750367 \backslash \mathrm{RMSD}=6$ $.432 \mathrm{e}-09 \backslash \mathrm{RMSF}=1.039 \mathrm{e}-05 \backslash \mathrm{Dipole}=0,0 .,-0.434059 \backslash \mathrm{PG}=\mathrm{C} 02$ [ $22(\mathrm{C} 1 \mathrm{C} 1 \mathrm{C} 1 \mathrm{H} 1), \mathrm{X}($ C6H10S2 ) $] \backslash \backslash @$

\section{$\mathrm{CH}_{3} \mathrm{SCH}(\mathrm{Ph}) \mathrm{S}-\mathrm{CH}_{3}$}

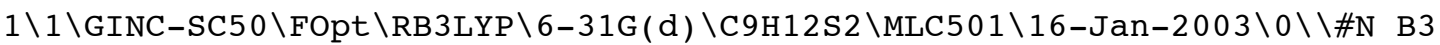
LYP /6-31G(D) OPT FREQ MAXDISK=39321600 \\ralk $z=p h \quad r=$ me-c2-6dub3 $\backslash \backslash 0,1 \backslash \mathrm{C}$ $,-0.58192849,-0.0676167262,0.6559765072 \backslash C, 0 . \overline{8} 6926 \overline{5} 9739,0.0219992291,0$. $1701840044 \backslash \mathrm{C}, 1.4857483314,1.2596861061,-0.0639221236 \backslash \mathrm{C}, 1.6453478345,-1$ $.1399199407,0.0253093128 \backslash \mathrm{C}, 2.827536998,1.3344024726,-0.4400288414 \backslash \mathrm{C}, 2$. $9867423773,-1.0640643596,-0.350976908 \backslash \mathrm{H}, 0.912625679,2.1721030051,0.063$ $0616357 \backslash \mathrm{H}, 1.20024715,-2.1111659146,0.2126653432 \backslash \mathrm{H}, 3.2788869284,2.30745$ $02028,-0.6159236246 \backslash \mathrm{H}, 3.56335567,-1.9793611551,-0.4563357395 \backslash \mathrm{C}, 3.58498$ $42664,0.1731079472,-0.58918729 \backslash \mathrm{H}, 4.6290560015,0.2314056401,-0.88493589$ $19 \backslash \mathrm{S},-1.4018208475,-1.5981623652,-0.0019989974 \backslash \mathrm{C},-2.8407743157,-1.6904$ $387431,1.1164454541 \backslash \mathrm{H},-3.4397390754,-2.5420528156,0.7830438314 \backslash \mathrm{H},-2.52$ $85067457,-1.8596510317,2.1517464095 \backslash \mathrm{H},-3.4396520659,-0.7786431009,1.05$ $24807841 \backslash \mathrm{S},-1.6058304669,1.4537346691,0.4124901549 \backslash \mathrm{C},-1.6926480643,1.5$ $529707235,-1.4095722981 \backslash \mathrm{H},-2.3798197454,2.3703902428,-1.6429581939 \backslash \mathrm{H},-$ $0.7131453282,1.7672784907,-1.8446896079 \backslash \mathrm{H},-2.0852459047,0.6192369939$,$1.8199378508 \backslash \mathrm{H},-0.5812910017,-0.1669076734,1.7485574803 \backslash \backslash$ Version=DEC-A 
$\mathrm{XP}-\mathrm{OSF} / 1-\mathrm{G} 98 \mathrm{R}$ evA. $11.3 \backslash \mathrm{HF}=-1146.5571283 \backslash \mathrm{RMSD}=4.678 \mathrm{e}-09 \backslash \mathrm{RMSF}=3.686 \mathrm{e}-06 \backslash \mathrm{D}$ ipole $=0.0197242,-0.0250547,0.0113779 \backslash \mathrm{PG}=\mathrm{C} 01 \quad[\mathrm{X}(\mathrm{C} 9 \mathrm{H} 12 \mathrm{~S} 2)] \backslash \backslash @$

\section{$\mathrm{S}=\mathrm{C}\left(\mathrm{CH}_{3}\right)_{2}$}

$1 \backslash 1 \backslash G I N C-S C 62 \backslash F O p t \backslash R B 3 L Y P \backslash 6-31 G(d) \backslash C 3 H 6 S 1 \backslash M L C 501 \backslash 22-F e b-2002 \backslash 1 \backslash \backslash \# N$ B3L YP $/ 6-31 \mathrm{G}$ * FOPT $=($ TIGHT, Z-MATRIX) SCF=TIGHT FREQ MAXDISK $=3932160000$ GEOM $=$ CHECK GUESS $=\mathrm{READ} \backslash \backslash \mathrm{S}=\mathrm{C}(\mathrm{CH} 3) 2$ in $\mathrm{C} 2 \mathrm{v}-\mathrm{in} \backslash \backslash 0,1 \backslash \mathrm{S} \backslash \mathrm{C}, 1, \mathrm{~B} 1 \backslash \mathrm{C}, 2, \mathrm{~B} 2,1, \mathrm{~A} 1 \backslash \mathrm{H}, 3$, $\mathrm{B} 3,2, \mathrm{~A} 2,1,0 ., 0 \backslash \mathrm{H}, 3, \mathrm{~B} 4,2, \mathrm{~A} 3,4, \mathrm{D} 2,0 \backslash \mathrm{H}, 3, \mathrm{~B} 4,2, \mathrm{~A} 3,4,-\mathrm{D} 2,0 \backslash \mathrm{C}, 2, \mathrm{~B} 2,1, \mathrm{~A} 1,3,18$ 0 . $, 0 \backslash \mathrm{H}, 7, \mathrm{~B} 3,2, \mathrm{~A} 2,1,0 ., 0 \backslash \mathrm{H}, 7, \mathrm{~B} 4,2, \mathrm{~A} 3,8, \mathrm{D} 2,0 \backslash \mathrm{H}, 7, \mathrm{~B} 4,2, \mathrm{~A} 3,8,-\mathrm{D} 2,0 \backslash \backslash \mathrm{B} 1=1.6$ $3874106 \backslash \mathrm{B} 2=1.50753656 \backslash \mathrm{B} 3=1.09063254 \backslash \mathrm{B} 4=1.0992076 \backslash \mathrm{A} 1=122.55208605 \backslash \mathrm{A} 2=11$ $1.83735755 \backslash A 3=110.04785298 \backslash \mathrm{D} 2=-121.60138385 \backslash \backslash$ Version=DEC-AXP-OSF $/ 1-\mathrm{G} 98$ RevA.9 $\backslash$ State $=1-A 1 \backslash H F=-516.1101889 \backslash \mathrm{RMSD}=5.683 \mathrm{e}-09 \backslash \mathrm{RMSF}=1.122 \mathrm{e}-06 \backslash \mathrm{Dipole}$ $=0 ., 0 ., 1.2003336 \backslash \mathrm{PG}=\mathrm{C} 02 \mathrm{~V}[\mathrm{C} 2(\mathrm{C} 1 \mathrm{~S} 1), \mathrm{SGV}(\mathrm{C} 2 \mathrm{H} 2), \mathrm{X}(\mathrm{H} 4)] \backslash \backslash @$

\section{TS: $\cdot \mathrm{CH}_{3}+\mathrm{S}=\mathrm{C}\left(\mathrm{CH}_{3}\right)_{2}$}

$1 \backslash 1 \backslash G I N C-S C 22 \backslash F T S \backslash U B 3 L Y P \backslash 6-31 G(d) \backslash C 4 H 9 S 1(2) \backslash M L C 501 \backslash 12-M a r-2002 \backslash 1 \backslash \backslash \# N \quad U$ $\mathrm{B} 3 \mathrm{LYP} / 6-31 \mathrm{G}(\mathrm{D}) \mathrm{OPT}=(\mathrm{TS}, \mathrm{Z}-\mathrm{MATRIX}, \mathrm{EF}, \mathrm{MAXCYC}=250, \mathrm{TIGHT})$ FREQ MAXDISK=6553 $60000 \mathrm{GEOM}=\mathrm{CHECK}$ GUESS=READ SCF=TIGHT $\backslash \backslash \mathrm{CH} 3-\mathrm{S}-\mathrm{C}(\mathrm{CH} 2) 2 * \mathrm{C} 1 \backslash \backslash 0,2 \backslash \mathrm{S} \backslash \mathrm{C}, 1, \mathrm{~B} 1$ $\backslash \mathrm{C}, 2, \mathrm{~B} 2,1, \mathrm{~A} 1 \backslash \mathrm{C}, 2, \mathrm{~B} 3,1, \mathrm{~A} 2,3, \mathrm{D} 1,0 \backslash \mathrm{C}, 1, \mathrm{~B} 4,2, \mathrm{~A} 3,3, \mathrm{D} 2,0 \backslash \mathrm{H}, 5, \mathrm{~B} 5,1, \mathrm{~A} 4,2, \mathrm{D} 3,0 \backslash$ $\mathrm{H}, 5, \mathrm{~B} 6,1, \mathrm{~A} 5,6, \mathrm{D} 4,0 \backslash \mathrm{H}, 5, \mathrm{~B} 7,1, \mathrm{~A} 6,6, \mathrm{D} 5,0 \backslash \mathrm{H}, 3, \mathrm{~B} 8,2, \mathrm{~A} 7,1, \mathrm{D} 6,0 \backslash \mathrm{H}, 3, \mathrm{~B} 9,2, \mathrm{~A} 8,1$ , $\mathrm{D} 7,0 \backslash \mathrm{H}, 3, \mathrm{~B} 10,2, \mathrm{~A} 9,1, \mathrm{D} 8,0 \backslash \mathrm{H}, 4, \mathrm{~B} 11,2, \mathrm{~A} 10,1, \mathrm{D} 9,0 \backslash \mathrm{H}, 4, \mathrm{~B} 12,2, \mathrm{~A} 11,1, \mathrm{D} 10,0 \backslash \mathrm{H}$ $, 4, \mathrm{~B} 13,2, \mathrm{~A} 12,1, \mathrm{D} 11,0 \backslash \backslash \mathrm{B} 1=1.64466579 \backslash \mathrm{B} 2=1.50776015 \backslash \mathrm{B} 3=1.50776006 \backslash \mathrm{B} 4=2.8$ $6336834 \backslash \mathrm{B} 5=1.0827206 \backslash \mathrm{B} 6=1.08241299 \backslash \mathrm{B} 7=1.08241331 \backslash \mathrm{A} 1=122.50653995 \backslash \mathrm{A} 2=12$ $2.50656636 \backslash A 3=114.45095224 \backslash A 4=90.60079387 \backslash A 5=95.89546473 \backslash A 6=95.8967954$ $4 \backslash D 1=-181.34064566 \backslash D 2=-89.33197972 \backslash D 3=-179.98207565 \backslash D 4=-119.59616051 \backslash D$ $5=119.59587769 \backslash \mathrm{B} 8=1.09097995 \backslash \mathrm{B} 9=1.09943272 \backslash \mathrm{B} 10=1.09998335 \backslash \mathrm{B} 11=1.099432$ $56 \backslash B 12=1.09097998 \backslash B 13=1.09998355 \backslash A 7=111.82149357 \backslash A 8=110.27130144 \backslash A 9=11$ $0.25501058 \backslash A 10=110.27147556 \backslash A 11=111.82148956 \backslash A 12=110.25484146 \backslash D 6=-2.02$ $894317 \backslash D 7=-123.58072077 \backslash D 8=119.42927267 \backslash D 9=123.58346281 \backslash D 10=2.03140961$ $\backslash \mathrm{D} 11=240.57345204 \backslash \backslash$ Version=DEC-AXP-OSF $/ 1-G 98 \mathrm{RevA} .9 \backslash \mathrm{HF}=-555.9480428 \backslash \mathrm{S} 2=$ $0.769411 \backslash \mathrm{S} 2-1=0 . \backslash \mathrm{S} 2 \mathrm{~A}=0.750127 \backslash \mathrm{RMSD}=8.389 \mathrm{e}-09 \backslash \mathrm{RMSF}=1.422 \mathrm{e}-05 \backslash \mathrm{Dipole}=0.0$ $032467,0.2783954,0.886936 \backslash \mathrm{PG}=\mathrm{C} 01 \quad[\mathrm{X}(\mathrm{C} 4 \mathrm{H} 9 \mathrm{~S} 1)] \backslash \backslash @$

\section{$\mathrm{CH}_{3} \mathrm{SC} \cdot\left(\mathrm{CH}_{3}\right)_{2}$}

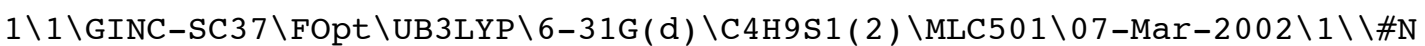
UB3LYP $/ 6-31 G *$ FOPT $=(Z-M A T R I X) \quad S C F=T I G H T$ FREQ MAXDISK=3932160000 GEOM=C $\mathrm{HECK} \backslash \backslash \mathrm{CH} 3-\mathrm{S}-{ }^{*} \mathrm{C}(\mathrm{CH} 3) 2$ in $\mathrm{C} 1 \mathrm{symm} \backslash \backslash 0,2 \backslash \mathrm{S} \backslash \mathrm{C}, 1, \mathrm{~B} 1 \backslash \mathrm{C}, 2, \mathrm{~B} 2,1, \mathrm{~A} 1 \backslash \mathrm{C}, 2, \mathrm{~B} 3,1, \mathrm{~A} 2$, $3, \mathrm{D} 1,0 \backslash \mathrm{C}, 1, \mathrm{~B} 4,2, \mathrm{~A} 3,3, \mathrm{D} 2,0 \backslash \mathrm{H}, 5, \mathrm{~B} 5,1, \mathrm{~A} 4,2, \mathrm{D} 3,0 \backslash \mathrm{H}, 5, \mathrm{~B} 6,1, \mathrm{~A} 5,6, \mathrm{D} 4,0 \backslash \mathrm{H}, 5, \mathrm{~B} 7$ $, 1, \mathrm{~A} 6,6, \mathrm{D} 5,0 \backslash \mathrm{H}, 3, \mathrm{~B} 8,2, \mathrm{~A} 7,1, \mathrm{D} 6,0 \backslash \mathrm{H}, 3, \mathrm{~B} 9,2, \mathrm{~A} 8,1, \mathrm{D} 7,0 \backslash \mathrm{H}, 3, \mathrm{~B} 10,2, \mathrm{~A} 9,1, \mathrm{D} 8,0$ $\backslash \mathrm{H}, 4, \mathrm{~B} 11,2, \mathrm{~A} 10,1, \mathrm{D} 9,0 \backslash \mathrm{H}, 4, \mathrm{~B} 12,2, \mathrm{~A} 11,1, \mathrm{D} 10,0 \backslash \mathrm{H}, 4, \mathrm{~B} 13,2, \mathrm{~A} 12,1, \mathrm{D} 11,0 \backslash \backslash \mathrm{B} 1=$ $1.76040029 \backslash \mathrm{B} 2=1.50116469 \backslash \mathrm{B} 3=1.49930006 \backslash \mathrm{B} 4=1.82899497 \backslash \mathrm{B} 5=1.09299456 \backslash \mathrm{B} 6=$ $1.09335041 \backslash \mathrm{B} 7=1.09272043 \backslash \mathrm{A} 1=114.32311171 \backslash \mathrm{A} 2=121.81593508 \backslash \mathrm{A} 3=104.030368$ $33 \backslash A 4=106.04568798 \backslash A 5=110.89519235 \backslash A 6=111.94271848 \backslash D 1=-154.0787177 \backslash D 2=$ $-169.04426525 \backslash D 3=-186.43130151 \backslash D 4=-118.19677541 \backslash D 5=118.46758739 \backslash B 8=1.0$ $9556304 \backslash \mathrm{B} 9=1.10437127 \backslash \mathrm{B} 10=1.09829993 \backslash \mathrm{B} 11=1.10516989 \backslash \mathrm{B} 12=1.09504758 \backslash \mathrm{B} 13$ $=1.0974191 \backslash \mathrm{A} 7=111.97869626 \backslash \mathrm{A} 8=112.51774977 \backslash \mathrm{A} 9=110.25141341 \backslash \mathrm{A} 10=112.165$ $31975 \backslash A 11=113.04786072 \backslash A 12=109.97870399 \backslash D 6=34.59578555 \backslash D 7=-86.38557568$ $\backslash \mathrm{D} 8=154.77050823 \backslash \mathrm{D} 9=77.19465282 \backslash \mathrm{D} 10=-43.91014241 \backslash \mathrm{D} 11=195.9695628 \backslash \backslash$ Vers ion=DEC-AXP-OSF $/ 1-G 98 R e v A .9 \backslash \mathrm{HF}=-555.9879304 \backslash \mathrm{S} 2=0.754442 \backslash \mathrm{S} 2-1=0 . \backslash \mathrm{S} 2 \mathrm{~A}=0$. $750015 \backslash \mathrm{RMSD}=4.175 \mathrm{e}-09 \backslash \mathrm{RMSF}=1.747 \mathrm{e}-05 \backslash \mathrm{Dipole}=-0.5112718,-0.0022178,0.22$ $33791 \backslash \mathrm{PG}=\mathrm{C} 01 \quad[\mathrm{X}(\mathrm{C} 4 \mathrm{H} 9 \mathrm{~S} 1)] \backslash \backslash @$

\section{$\mathrm{CH}_{3} \mathrm{SCH}\left(\mathrm{CH}_{3}\right)_{2}$}

$1 \backslash 1 \backslash G I N C-S C 81 \backslash F O p t \backslash R B 3 L Y P \backslash 6-31 G(d) \backslash C 4 H 10 S 1 \backslash M L C 501 \backslash 30-M a y-2005 \backslash 0 \backslash \backslash \#$ B3L

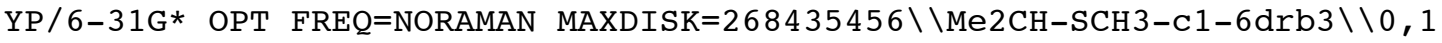
$\backslash S, 0.7336720854,-0.7252505216,-0.3768356797 \backslash \mathrm{C},-0.7123226234,0.13750487$ $5,0.3938031119 \backslash \mathrm{C},-1.9396155541,-0.7564106069,0.1892568743 \backslash \mathrm{C},-0.9328839$ $072,1.5431085302,-0.1710472726 \backslash \mathrm{C}, 2.1247273994,0.2794397435,0.250674603$ $2 \backslash \mathrm{H}, 3.0422441345,-0.214108602,-0.0808328808 \backslash \mathrm{H}, 2.1239925956,0.315397068$ $3,1.3449123881 \backslash \mathrm{H}, 2.1129623379,1.2967419209,-0.1507420476 \backslash \mathrm{H},-1.79668237$ $64,-1.7429766067,0.6410347149 \backslash \mathrm{H},-2.1506327666,-0.8956533651,-0.8775986$ $903 \backslash \mathrm{H},-2.8214636085,-0.2922841131,0.6466743783 \backslash \mathrm{H},-1.1466511644,1.50055$ 
$65197,-1.2446863426 \backslash \mathrm{H},-0.0594830002,2.1867721431,-0.0241614988 \backslash \mathrm{H},-1.78$ $4280776,2.0220224982,0.3303915884 \backslash \mathrm{H},-0.4981906303,0.2056856309,1.46825$ $53644 \backslash \backslash$ Version=Al64 T-G03RevC.02 \State $=1-\mathrm{A} \backslash \mathrm{HF}=-556.6427746 \backslash \mathrm{RMSD}=8.029 \mathrm{e}-$ $09 \backslash \mathrm{RMSF}=1.104 \mathrm{e}-05 \backslash \mathrm{Dipole}=-0.0743159,0.5332441,0.4079326 \backslash \mathrm{PG}=\mathrm{C} 01 \quad[\mathrm{X}(\mathrm{C} 4 \mathrm{H} 1$ $0 \mathrm{~S} 1)] \backslash \backslash @$

\section{For Bond Strength Calculations:}

$\mathrm{H}_{2}$

$1 \backslash 1 \backslash G I N C-S C 97 \backslash F O p t \backslash R B 3 L Y P \backslash 6-31 G(d) \backslash H 2 \backslash M L C 501 \backslash 01-J u n-2005 \backslash 0 \backslash \backslash \#$ B3LYP/6$31 \mathrm{G} *$ OPT $=$ TIGHT FREQ MAXDISK $=134217728 \backslash \backslash \mathrm{H} 2 \backslash \backslash 0,1 \backslash \mathrm{H}, 0,0,,-0.3713940648 \backslash \mathrm{H}$ , $0 ., 0.0 .3713940648 \backslash \backslash$ Version $=\mathrm{A} 164 \mathrm{~T}-\mathrm{G} 03$ RevC.02 $\backslash$ State $=1-\mathrm{SGG} \backslash \mathrm{HF}=-1.175482$ $4 \backslash \mathrm{RMSD}=1.899 \mathrm{e}-12 \backslash \mathrm{RMSF}=5.952 \mathrm{e}-09 \backslash \mathrm{Dipole}=0,0 ., 0 . \backslash \mathrm{PG}=\mathrm{D} * \mathrm{H} \quad[\mathrm{C} *(\mathrm{H} 1 . \mathrm{H} 1)] \backslash \backslash @$

\section{H・}

$1 \backslash 1 \backslash G I N C-S C 30 \backslash S P \backslash U B 3 L Y P \backslash 6-31 G(d) \backslash H 1(2) \backslash M L C 501 \backslash 24-O c t-2003 \backslash 0 \backslash \backslash \#$ B3LYP / 6

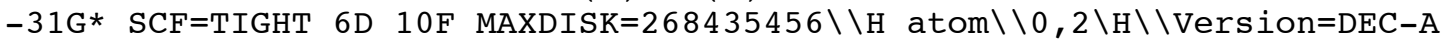
$\mathrm{XP}-\mathrm{OSF} / 1-\mathrm{G} 03 \mathrm{RevB} .03 \backslash \mathrm{State}=2-\mathrm{A} 1 \mathrm{G} \backslash \mathrm{HF}=-0.5002728 \backslash \mathrm{S} 2=0.75 \backslash \mathrm{S} 2-1=0 . \backslash \mathrm{S} 2 \mathrm{~A}=0.75$ $\backslash \mathrm{RMSD}=5.069 \mathrm{e}-12 \backslash \mathrm{Dipole}=0 ., 0 ., 0 . \backslash \mathrm{PG}=\mathrm{OH}[\mathrm{O}(\mathrm{H} 1)] \backslash \backslash @$

\section{HS- $\mathrm{CH}\left(\mathrm{CH}_{3}\right) \mathrm{SCH}_{3}$}

$1 \backslash 1 \backslash G I N C-A C 3 \backslash F O p t \backslash R B 3 L Y P \backslash 6-31 G(d) \backslash C 3 H 8 S 2 \backslash M L C 501 \backslash 03-J u n-2005 \backslash 0 \backslash \backslash \#$ B 3LYP /6-31G* OPT FREQ=NORAMAN MAXDISK=500MB \\HSCHMeSCH3-c1b-6dub3 $\backslash \backslash 0,1 \backslash \mathrm{S},-1$ $.5130230671,0.7813986697,-0.3937399676 \backslash \mathrm{C}, 0.1249384973,0.5546207244,0.4$ $608555336 \backslash \mathrm{C}, 1.0030279194,1.8022215684,0.3755686434 \backslash \mathrm{S}, 1.0145466221,-0.8$ $524366586,-0.3059198534 \backslash \mathrm{C}, 0.0257745507,-2.2629740138,0.3036147525 \backslash \mathrm{H}, 0$. $4720014345,-3.1635241525,-0.1263983638 \backslash \mathrm{H},-1.0130316443,-2.1840393808,-$ $0.0251593135 \backslash \mathrm{H}, 0.0686436485,-2.3329456563,1.3947415702 \backslash \mathrm{H}, 0.4864408841$, $2.6613298102,0.8161717058 \backslash \mathrm{H}, 1.9416136153,1.6449634424,0.9191596336 \backslash \mathrm{H}, 1$ $.234632284,2.0459366618,-0.6657446482 \backslash \mathrm{H},-0.0781774926,0.3007584237,1.5$ $054706819 \backslash \mathrm{H},-2.0589454143,1.6009189997,0.5360822941 \backslash \backslash$ Version=IA64L-G03 RevC. 02 \State $=1-A \backslash H F=-915.5099557 \backslash R M S D=3.540 e-09 \backslash R M S F=1.530 e-05 \backslash D i p o l e$ $=-0.07484,0.0395365,0.8658248 \backslash P G=C 01[X(C 3 H 8 S 2)] \backslash \backslash @$

\section{$\mathrm{HS}-\mathrm{C} \cdot\left(\mathrm{CH}_{3}\right) \mathrm{SCH}_{3}$}

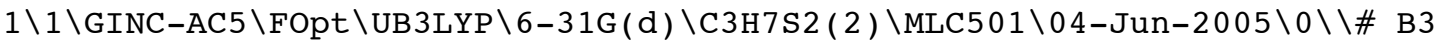
LYP / 6-31G* OPT FREQ=NORAMAN MAXDISK $=268435456 \backslash \backslash$ HSCMeSCH3-cla-6dub3 $\backslash \backslash 0$, $2 \backslash \mathrm{S},-1.2291373188,-1.2039985223,-0.1182203422 \backslash \mathrm{C},-0.4927596915,0.397564$ $4737,0.1250655047 \backslash \mathrm{C},-1.3761437205,1.6103045211,0.1791258325 \backslash \mathrm{S}, 1.171537$ $6349,0.6233880967,-0.3772368361 \backslash C, 2.0632341953,-0.7414859777,0.4653752$ $187 \backslash \mathrm{H}, 3.1165401672,-0.6211673644,0.1984559394 \backslash \mathrm{H}, 1.7114110303,-1.716145$ $7065,0.1232076361 \backslash \mathrm{H}, 1.9474348947,-0.660789441,1.5484915886 \backslash \mathrm{H},-2.197091$ $4609,1.4608456705,0.8937518052 \backslash \mathrm{H},-0.8057731757,2.4889103546,0.49778980$ $13 \backslash \mathrm{H},-1.8393786903,1.8371742558,-0.7940079158 \backslash \mathrm{H},-2.1775325218,-1.09735$ $90615,0.8422266623 \backslash \backslash$ Version $=I A 64 \mathrm{~L}-\mathrm{G} 03$ RevC. $02 \backslash$ State $=2-\mathrm{A} \backslash \mathrm{HF}=-914.8605517$ $\backslash \mathrm{S} 2=0.755027 \backslash \mathrm{S} 2-1=0 . \backslash \mathrm{S} 2 \mathrm{~A}=0.750019 \backslash \mathrm{RMSD}=7.375 \mathrm{e}-09 \backslash \mathrm{RMSF}=8.913 \mathrm{e}-06 \backslash \mathrm{Dipole}$ $=-0.1032678,-0.0128086,0.5409518 \backslash P G=C 01[X(C 3 H 7 S 2)] \backslash \backslash @$

\section{-S- $\mathrm{CH}\left(\mathrm{CH}_{3}\right) \mathrm{SCH}_{3}$}

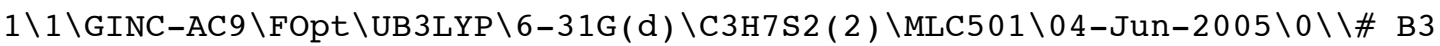

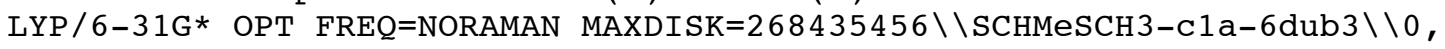
$2 \backslash S,-1.2673617708,-1.1256146594,-0.3298571682 \backslash \mathrm{C},-0.5446580189,0.335037$ $8809,0.4732751899 \backslash \mathrm{C},-1.3399716126,1.6258192716,0.3143494932 \backslash \mathrm{S}, 1.059833$ $4986,0.4471894325,-0.445593335 \backslash \mathrm{C}, 2.1031116507,-0.6907375194,0.53181403$ $5 \backslash \mathrm{H}, 3.0492693685,-0.7941604729,-0.0049813656 \backslash \mathrm{H}, 1.6231419482,-1.6685884$ $837,0.6076583561 \backslash \mathrm{H}, 2.2947100226,-0.2815516692,1.5277247733 \backslash \mathrm{H},-2.322394$ $4889,1.5090728137,0.7840411994 \backslash \mathrm{H},-0.82024004,2.4643152417,0.792589923 \backslash$ $\mathrm{H},-1.4998568108,1.8609603543,-0.7422879871 \backslash \mathrm{H},-0.3150697602,0.144038047$ , $1.5258308438 \backslash \backslash$ Version=IA64L-G03RevC.02 \State $=2-\mathrm{A} \backslash \mathrm{HF}=-914.877883 \backslash \mathrm{S} 2=0$. $753469 \backslash \mathrm{S} 2-1=0 . \backslash \mathrm{S} 2 \mathrm{~A}=0.750008 \backslash \mathrm{RMSD}=4.322 \mathrm{e}-09 \backslash \mathrm{RMSF}=6.336 \mathrm{e}-06 \backslash \mathrm{Dipole}=0.673$ $4381,0.4140557,0.8017532 \backslash P G=C 01[\mathrm{X}(\mathrm{C} 3 \mathrm{H} 7 \mathrm{~S} 2)] \backslash \backslash @$ 


\section{$\mathrm{HS}-\mathrm{PH}\left(\mathrm{CH}_{3}\right)_{2} \mathrm{SCH}_{3}$}

$1 \backslash 1 \backslash G I N C-A C 4 \backslash F O p t \backslash R B 3 L Y P \backslash 6-31 G(d) \backslash C 3 H 11 P 1 S 2 \backslash M L C 501 \backslash 02-J u n-2005 \backslash 0 \backslash \backslash \#$ B3 LYP/6-31G* OPT FREQ=NORAMAN MAXDISK=500MB \\HSPHMe2SCH3-C1a-6dub3 $\backslash \backslash 0,1 \backslash$ $\mathrm{P}, 0.0459034751,0.1337253702,-0.2369523221 \backslash \mathrm{C}, 1.8250692211,-0.1830687142$ $, 0.1182097492 \backslash \mathrm{C},-0.4304694002,1.9131993602,-0.2955031503 \backslash \mathrm{H},-0.78110463$ $35,-0.9748054632,-0.5136356546 \backslash S, 0.3009614326,-0.0107588684,-2.5776780$ $361 \backslash \mathrm{S},-0.7091756704,-0.0277804492,1.9273065286 \backslash \mathrm{C},-0.8528389814,-1.8471$ $172016,2.0741991022 \backslash \mathrm{H}, 0.2442392434,2.444529492,-0.9696875809 \backslash \mathrm{H},-0.4092$ $853314,2.348783928,0.7072462016 \backslash \mathrm{H},-1.4436843481,1.9736867316,-0.699306$ $1999 \backslash \mathrm{H}, 2.4499885677,0.3135182477,-0.6262817185 \backslash \mathrm{H}, 1.9940143906,-1.26081$ $52187,0.0407261615 \backslash \mathrm{H}, 2.0612058653,0.1509184596,1.1305687896 \backslash \mathrm{H}, 0.105147$ $7627,-2.3180193743,2.3131424782 \backslash \mathrm{H},-1.2488296526,-2.2880780266,1.149511$ $1993 \backslash \mathrm{H},-1.5606462913,-2.0628437208,2.8781698887 \backslash \mathrm{H}, 1.1812650678,0.98579$ $28065,-2.8316588203 \backslash \backslash$ Version=IA64L-G03RevC.02 $\backslash$ State $=1-A \backslash H F=-1258.65439$ $6 \backslash \mathrm{RMSD}=6.312 \mathrm{e}-09 \backslash \mathrm{RMSF}=9.812 \mathrm{e}-06 \backslash \mathrm{Dipole}=0.6160255,0.1920692,0.4776762 \backslash \mathrm{P}$ $\mathrm{G}=\mathrm{C} 01[\mathrm{X}(\mathrm{C} 3 \mathrm{H} 11 \mathrm{P} 1 \mathrm{~S} 2)] \backslash \backslash \mathrm{Q}$

\section{$\mathrm{HS}-\mathrm{P} \cdot\left(\mathrm{CH}_{3}\right)_{2} \mathrm{SCH}_{3}$}

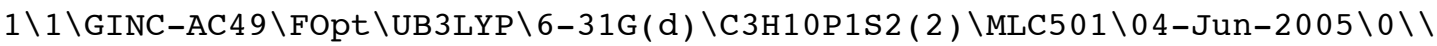

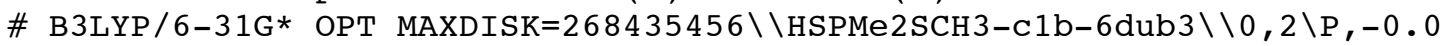
$012706456,0.2258867717,-0.2249618701 \backslash \mathrm{C}, 1.8347330308,0.4880282746,-0.19$ $18590669 \backslash \mathrm{C},-0.6563608659,1.9552240263,-0.3476979079 \backslash \mathrm{S},-0.063680544,-0$. $4414226322,-2.5021274782 \backslash \mathrm{S},-0.4914651985,-0.0549169235,1.964726746 \backslash \mathrm{C},-$ $0.1376722561,-1.8491125308,2.129549125 \backslash \mathrm{H},-0.277599776,2.4154338738,-1$. $2631184568 \backslash \mathrm{H},-0.3498282782,2.5492379555,0.5190648095 \backslash \mathrm{H},-1.748646699,1$. $9277949482,-0.3904730256 \backslash \mathrm{H}, 2.16680343,0.9521553822,-1.1254535654 \backslash \mathrm{H}, 2.3$ $399359849,-0.4757881258,-0.0830636605 \backslash \mathrm{H}, 2.0999789556,1.1287991081,0.65$ $26996836 \backslash \mathrm{H}, 0.9299713875,-2.0616429268,2.0311037475 \backslash \mathrm{H},-0.6998735875,-2$. $4269530037,1.3919291236 \backslash \mathrm{H},-0.4643460691,-2.1415819122,3.1306307709 \backslash \mathrm{H},-$ $1.3392032366,-0.8791626039,-2.4304325611 \backslash \backslash$ Version=IA64L-G03RevC.02\Sta $\mathrm{te}=2-\mathrm{A} \backslash \mathrm{HF}=-1258.0631587 \backslash \mathrm{S} 2=0.757238 \backslash \mathrm{S} 2-1=0 . \backslash \mathrm{S} 2 \mathrm{~A}=0.750029 \backslash \mathrm{RMSD}=8.418 \mathrm{e}-0$ $9 \backslash \mathrm{RMSF}=5.812 \mathrm{e}-06 \backslash \mathrm{Dipole}=0.2152853,0.1604005,0.4413627 \backslash \mathrm{PG}=\mathrm{C} 01 \quad[\mathrm{X}(\mathrm{C} 3 \mathrm{H} 10 \mathrm{P}$ $1 \mathrm{~S} 2)] \backslash \backslash @$

\section{$\cdot \mathrm{S}-\mathrm{PH}\left(\mathrm{CH}_{3}\right)_{2} \mathrm{SCH}_{3}$}

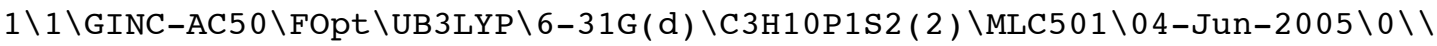
\# B3LYP/6-31G* OPT FREQ=NORAMAN MAXDISK=268435456\\SPHMe2SCH3-C $1 \mathrm{a}-6 \mathrm{dub}$ $3 \backslash \backslash 0,2 \backslash P, 0.3626563278,0.0696076929,0.041083554 \backslash \mathrm{C}, 0.3697394747,-0.70587$ $8433,1.7080656669 \backslash \mathrm{C}, 0.7236651181,1.8743145073,0.1181720485 \backslash \mathrm{H}, 0.0709896$ $192,-0.7936139045,-1.0605966704 \backslash S, 2.4011805483,-0.5598095218,-0.653773$ $9213 \backslash \mathrm{S},-1.9107844844,0.5334537575,-0.0599327496 \backslash \mathrm{C},-2.5559866221,-1.099$ $6111706,-0.5738744395 \backslash \mathrm{H}, 1.7074476785,2.0040336136,0.5782486542 \backslash \mathrm{H},-0.05$ $16520847,2.3996076694,0.6831355189 \backslash \mathrm{H}, 0.758733427,2.2624002433,-0.90221$ $61669 \backslash \mathrm{H}, 1.3726269757,-0.642464461,2.1371142382 \backslash \mathrm{H}, 0.1055975498,-1.76128$ $10272,1.5978552798 \backslash \mathrm{H},-0.3708913634,-0.2137418652,2.341989872 \backslash \mathrm{H},-2.6943$ $855951,-1.7724283809,0.2771120348 \backslash \mathrm{H},-1.887526826,-1.5765047789,-1.3035$ $766034 \backslash \mathrm{H},-3.521629145,-0.9413796949,-1.0601923882 \backslash \backslash$ Version=IA64L-G03Re $\mathrm{vC} .02 \backslash \mathrm{State}=2-\mathrm{A} \backslash \mathrm{HF}=-1258.0155586 \backslash \mathrm{S} 2=0.752909 \backslash \mathrm{S} 2-1=0 . \backslash \mathrm{S} 2 \mathrm{~A}=0.750006 \backslash \mathrm{RMSD}$ $=8.604 \mathrm{e}-09 \backslash \mathrm{RMSF}=2.204 \mathrm{e}-06 \backslash \mathrm{Dipole}=-0.3208407,-0.0452274,0.7050138 \backslash \mathrm{PG}=\mathrm{C} 0$ $1[\mathrm{X}(\mathrm{C} 3 \mathrm{H} 10 \mathrm{P} 1 \mathrm{~S} 2)] \backslash \backslash @$ 